\title{
Effect of Mesoscopic Misfit on Growth, Morphology, Electronic Properties and Magnetism of Nanostructures at Metallic Surfaces
}

\author{
Oleg O. Brovko a, Dmitry I. Bazhanov ${ }^{\mathrm{a}, \mathrm{b}}$, Holger L. Meyerheim ${ }^{\mathrm{a}}$, Dirk Sander ${ }^{\mathrm{a}}$, Valeri S. Stepanyuk ${ }^{\mathrm{a}}$, \\ Jürgen Kirschner ${ }^{a, c}$ \\ ${ }^{a}$ Max-Planck-Institut für Mikrostrukturphysik, Weinberg 2, 06120 Halle (Germany) \\ ${ }^{b}$ Faculty of Physics, Moscow State University, 119899 Moscow (Russia) \\ ${ }^{c}$ Institut für Physik, Martin-Luther Universität 06120 Halle (Germany)
}

\begin{abstract}
Stress and strain originating from mesoscopic misfit at interfaces can have diverse effects on the properties of surfaces and nanostructures thereon. We review the sources and consequences of mesoscopic misfit at metallic surfaces and elucidate various ways in which it affects growth, morphology, electronic properties and magnetism of thin films in early stages of epitaxy and epitaxial nanostructures.
\end{abstract}

Keywords: mesoscopic, misfit, relaxations, stress, strain, nanoscale

PACS: 68.35.-p, 68.65.-k, 73., 75.70.-i, 75.75.-c

\section{Contents}

\section{Introducing mesoscopic misfit}

2 Mesoscopic misfit - the history in brief

3 Concept of size-dependent mesoscopic relaxation and its effect on nanostructure growth and electronic/magnetic properties $\quad 4$

3.1 Hetero-epitaxial metal growth . . . . . . . 5

3.2 Homo-epitaxial metal growth . . . . . . . . 7

3.3 Size-dependent mesoscopic misfit . . . . . . 8

3.4 Size-dependent shape transitions in mesoscopic islands . . . . . . . . . . . 10

3.5 Adsorption, diffusion on and near mesoscopic islands ................. 11

3.6 Mesoscopic mismatch as a driving force for morphology modification at the atomic scale 16

3.7 Local variation of electronic properties in strained nano-islands . . . . . . . . . . . . 18

3.8 Influence of mesoscopic relaxation on the magnetism of nano-islands . . . . . . . . 20

4 Experimental confirmation of mesoscopic mismatch I: Surface x-ray diffraction 21

$4.1 \quad$ Historical Background and Theory . . . . . 22

4.2 SXRD analysis of the mesoscopic relaxations

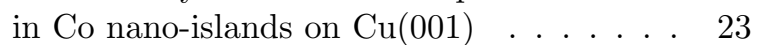

4.3 Mesoscopic misfit effect on the structure in the surfactant system $\mathrm{Fe} / \mathrm{O} / \mathrm{Fe}(001)-\mathrm{p}(1 \times 1)] 25$

Email address: obrovko@mpi-halle.de (Oleg O. Brovko)
5 Experimental confirmation of mesoscopic mismatch II: Surface extended x-ray absorption fine structure 28 5.1 Historical Background and Theory . . . . . 28 5.2 Experiment and data analysis . . . . . . . . . 29 5.3 Summary of the results . . . . . . . . 30

6 Experimental evidence for mesoscopic mismatch III: Monolayer-stress oscillations in hetero-epitaxy 31

6.1 Measurement of film stress with sub-monolayer sensitivity ............... 32

6.2 Stress oscillations with monolayer periodicity in Co layers on $\mathrm{Cu}(001)$. . . . . . . . . 33

6.3 Stress oscillations in other systems . . . . . 34 6.3.1 Stress oscillations in FeMn monolayers on $\mathrm{Cu}(001)$. . . . . . . . 35

7 Summary and conclusions

\section{Appendix A: Stress and strain defined}

\section{Introducing mesoscopic misfit}

Today, after more than a century of active research on the subject of stress and strain at surfaces, the notion that the latter play a crucial role in determining morphology and growth dynamics of both the surface itself and the nanostructures thereon seems almost trivial. To name just a few examples, we usually expect that the outermost atomic layers of a crystal cleaved along a high-symmetry crystal plane shall not retain the bulk interlayer spacing 
(a)

(b)

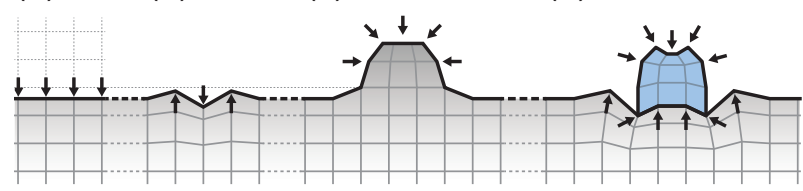

Figure 1: Cartoon representation of possible strain deformations of a surface: (a) clean, (b) clean reconstructed, (c,d) with homo- or hetero-epitaxially grown nanostructures.

but be contracted due to the reduced coordination of surface atoms (schematically shown in Fig. 1(a)). The equilibrium bond length contraction that causes this also introduces an excess stress in-plane of the crystal surface. Both excessive tensile and compressive stresses can lead to a reconstruction of a surface (hinted in Fig. 1(b)) as the latter strives to relieve some of the stress thus minimizing the surface energy. Contrary to flat surfaces, for finitesize nanoscale structures this in-plane stress is inherently uncompensated and leads to an in-plane deformation of the structure (Fig. 1(c)). While homo-epitaxial adsorption partially recovers the coordination of atoms at the surfacestructure interface ${ }^{1}$ in hetero-epitaxial systems (having differing equilibrium values of lattice constants and surface stresses) complex and highly anisotropic strain fields in both substrate and epitaxial nanostructure are often observed (Fig. 1(d)).

It is nowadays customary to generalize under the name of "mesoscopic misfit" the deformations of the surface and the nanostructures thereon induced by a multitude of effects connected with the finite size of the system or the inherent mismatch of lattice constants of different materials it is comprised of. With the present review we shall summarize and bring some order into the current understanding of how and when mesoscopic misfit arises in nanostructures at metallic surfaces and what implications it has for morphology, growth, electronic and magnetic properties of the system.

In the following, after a brief historical overview of the subject in Sec. 2 we shall outline the state-of-the-art understanding of the mesoscopic misfit in metallic systems (Sec. 3). We start with the most obvious consequence of the misfit - geometric relaxations in the system and their implications for morphology of homo- and heteroepitaxial systems as predicted by theory (Secs. 3.2 3.3 . Supporting the theoretical predictions with ample experimental observations we discuss the elementary processes of adatom adsorption and diffusion that underlie the observed growth dynamics and morphologies of mesoscopic systems (Secs. 3.4 3.6). Having established the fundamentals, we proceed to the discussion of the effects that misfit

\footnotetext{
${ }^{1}$ In fact, as shall be discussed in the following, even in the case of homo-epitaxy, the stress relief patterns are very much size-dependent and lead to considerable deformations of both the ad-structure and the substrate. Here, however, for the sake of simplicity we present a naive picture in the spirit of continuum elasticity theory.
}

(and resulting strain relasations in the system) can have on the system's electronic (Sec. 3.7) and magnetic (Sec. 3.8) properties.

While numerous indirect hints as to the omnipresence and importance of mesoscopic misfit exist, direct proofs of misfit-induced strain are scarce. That is why, in the last few sections of the review we summarize the existing direct evidence of such strain as provided by surface x-ray diffraction (Sec. 4), extended x-ray adsorption fine structure (Secs. 5) and stress measurement (Sec. 6) techniques.

The review is rounded up by a short summary.

\section{Mesoscopic misfit - the history in brief}

While our to-date understanding of the possible origins of mesoscopic misfit still cannot be deemed absolute, the idea itself that strain and stress of a surface play a fundamental role in defining the properties of the latter is more than a century old, dating back to the works of Gibbs. 1, 2] Theoretically conceived and originally applicable on a macroscopic level to an interface between any two "phases" of matter, it is much older than the interest of the community in the atomic structure of the interfaces. In the early $20^{\text {th }}$ century, during the boom of crystallography, when a crystalline material A was grown onto a crystal surface of material B (a process for which the term "epitaxy" was coined by L. Royer [3]) the stress at the interface of $\mathrm{A}$ and $\mathrm{B}$ was usually considered to come from the "natural misfit" alone (the difference in the lattice constants of $\mathrm{A}$ and B). At that time the only important implication of stress was its effect on morphology and growth behavior of epitaxial systems. The understanding that stress was an excess quality that the surface will strive to relieve in order to minimize its energy [4, 5] called for a good predictive theory that would accurately take into account the roles of stress and strain on surface energy. Based on the definition of stress and strain given by Gibbs, [1, 2] Shuttleworth, [6] Herring [5] and others [7] have developed a robust theoretical description of stresses and strains at surfaces showing, among other things, that the latter can be highly anisotropic and inhomogeneous. Still, in the early days of epitaxy, people in the "growing" community tried to avoid misfit, perceiving it as a hindrance in the quest of creating perfect atomic interfaces. Only later it was realized, that stress can also affect many "interesting" properties of surfaces, e.g. not only their morphology, but also their electronic [8] and magnetic [9, 10, properties.

Aside from the crystal growth, a very solid fundament for our understanding of surface processes on the atomic level was laid by the "chemical" and "cluster" communities. In late 1920-s Davisson and Germer have reported observing a discrepancy between x-ray end electron scattering measurements of $\mathrm{Ni}$ clusters, speculating that it might result from a lattice contraction at the surface of as much as 30\%. 11] Lennard-Jones and Dent, motivated by these results, attempted to calculate the contractions of interatomic distances at a crystal boundary, [12] pointing 
out that it can be caused by electrostatic forces. While their calculations have shown that even for polar surfaces of ionic crystals the contraction is unlikely to exceed $5 \%$ (being yet smaller for non-polar ones) and thus the observations of Davisson and Germer must have a different explanation, the authors put forth, as a conclusion, three remarkable statements: (i) atomic-scale physics can lead to equilibrium bond contraction at the surface of a crystal; (ii) the component of the resulting stress which is normal to the surface of a crystal shall lead to a contraction of interlayer spacings at the surface; (iii) the mismatch between the equilibrium bond length at the surface and in the bulk shall result in the surface being tensile-stressed. All three of those conclusions were extremely novel at that time and arguably one of the first attempts of atomistic treatment of surface stress and strain. For the observations of Davisson and Germer a more feasible explanation was offered by Davisson and Germer themselves, [13, 14 as well as by Lennard-Jones and Dent, hinging on the fact that electrons can be refracted in the crystal in an optical sense with an index of refraction greater than unity. [15, 16]

From our modern perspective the turning point in the atomistic understanding of processes at surfaces came with Pauling publishing in 1929 his paper in which he has set a framework of principles governing the structure of complex ionic crystals, [17 based upon the analysis of the arrangement of anions about each cation in terms of polyhedrons. A few years later his continued research on the subject culminated in a seminal paper 18 establishing, based on quantum mechanical considerations, a set of more general rules regarding electron-pair bonds, dealing particularly with the strength of bonds in relation to the nature of the single-electron eigenfunctions involved. One general notion which can be derived from Pauling's rules and which shall be of particular importance to us can be summarized in simple words as "The fewer bonds an atom has, the stronger each one of those is". This notion formally proven by Pauling [19] for metals in form of an equation linking the covalent radius (metallic radius) of an atom with the change in bond number (or in coordination number, if the valence remains constant). In the following we shall see that this notion can provide, with few exceptions, an explanation for a majority of mesoscopics-related effects.

Historically, however, the link between the atomic scale picture and experimental observations was not made until much later. Only in the 1950-s, when high resolution electron diffraction experiments 20 23 have proven beyond doubt that lattice contraction in small metallic crystals is real and furthermore size-dependent, did the theoretical prediction put forth by Lennard-Jones, Dent and Pauling become the prevalent explanation for this sort of experimental observations. Reliable experimental determination of lattice contraction has also made it possible to extract the values of surface stress for various materials. [22, 23] Notably, however, by modern standards, those values were estimations at best, as the elastic anisotropy was generally neglected.
In late 1960-s Vermaak and Mays have made the first attempt to finally bring some order into the numerous (up to then controversial) definitions of surface "stress", "strain" and "free energy", [24, 25] adopting and adapting the definitions by Gibbs, Shuttleworth and Herring [1, 2, 5, 6] and drawing a clear distinction between the former two (stress and strain) as pertaining to elastic and plastic deformations, respectively. While their definitions deviate from the ones common today, ${ }^{2}$ their work can be said to have contributed to laying the boundary between the thermodynamic and the solid-state/crystal views of surface phenomena. ${ }^{3}$

The next qualitative evolutionary leap in our understanding of mesoscopic misfit came, as it often is, with the development of novel experimental techniques. Especially the emergence of local probe methods, such as the scanning tunneling microscopy (STM), 26] allowing for the investigation of surface properties at a truly atomic scale, the realization dawned [27, 28] that both the origins of mesoscopic misfit and its possible consequences were yet by far not fully appreciated. So while one part of the community continued, with sustained interest, studying the layer-wise grown epitaxial systems from the macroscopic perspective, 29 more and more groups turned their attention to finite mesoscopic-size structures [28, 30, 31] It was perceived, that even light-element atoms can, upon adsorption on a surface, cause complicated shifts of surrounding surface atoms. 32. This was a clear hint, that bonding properties at the atomic scale are pivotal in determining the local forces and hence the morphology of the system. Atomistic view of the misfit-related phenomena also brought a new perspective on growth modes. The origins of text-book growth classification into categories like Frank-van-der-Merve (2D), Volmer-Weber (3D) and Stranski-Krastanov (mixed) 33. were understood in more details, taking into account the effect of strain on the exact shape of epitaxially growing islands 34,35

Crucial insights also came from theory. Calculations with molecular static methods [36, 37] have shown that firstly, not only the stress and strain of ad-structures are important for the determination of their shape and growth patterns, but also the deformation of the substrate is a strain relief mechanism having a crucial effect on the process of epitaxy and its energetics. 36, 38. Secondly, stress and strain were shown to be very much dependent on the local environment (i.e. ad-structure shape and size). Thus finally the paramount importance of considering misfit "locally" or "mesoscopically" as opposed to relying, in the spirit of continuum elasticity theory, 39] on bulk values of "natural" misfit, was unambiguously established. [36, 40.

\footnotetext{
${ }^{2}$ See Appendix A for a short note on the definitions used in the present review.

${ }^{3}$ It should be noted that the original formulations by Gibbs 1, 2 already contained the distinction between the surface free energy and surface stress of a solid, in the sense that, in general, a different amount of reversible work is required to form unit surface than to increase a large surface by unit area through reversibly stretching it.
} 
A third, not least important, input of the theory was pointing out that mesoscopic misfit is not an exclusive property of hetero-epitaxy, 30, 41,44] but exists in homoepitaxial systems as well, 37] a notion later proven experimentally [45, 46] and unifying the understanding of "mesoscopic misfit" for all systems regardless of their chemistry.

As more attention was now also allotted to other consequences of mesoscopic misfit than merely morphology, ways to deliberately introduce surface deformations locally were sought and devised. Gsell, for example, has used gas bubbles under the surface to introduce stress into the material and study the interplay of the latter with adsorption of light elements. 47. In that topic, an important contribution was made by theoretical studies as well. Especially the density functional theory (DFT) had by that time sufficiently evolved, aided by the computational power of modern computers, to make possible large scale investigations of the effect of stress and strain on, e.g., reactivity of metallic surfaces. [48] Perhaps the most important advantage of the theoretical approach to the problem of mesoscopic misfit lies in the fact, that most experimental methods are only able to detect the consequences of the misfit, while theoretical methods, even semi-empirical ones, are able to give a truly local, atomically resolved, perspective on the geometric, electronic and magnetic properties of the system, leaving little margin for mistakes and misinterpretations. [36, 43, 44, 49].

In the following decade, numerous theoretical and experimental studies have been aimed at understanding in detail the exact origins of mesoscopic misfit [50] and hunting down particular examples of effects it has on morphology, growth dynamics, electronic and magnetic properties of surfaces and nanostructures thereon [36, 43, 44, 46, 4972

In the present review we shall address the main sources of mesoscopic misfit and its effects on the above-mentioned properties of epitaxial nanostructures on metallic surfaces, a class of systems which has arguably received the largest share of interest in the community.

Yet before we proceed, there is one matter of definition, which has to be resolved. In view of the evolution of the concept of mesoscopic misfit it is nowadays somewhat problematic to say exactly what meaning the term "mesocopic" has in this particular case. As we shall see in the following, the effects of the mesoscopic misfit can be traced on length scales ranging from 100-s of nanometers to a few Anogström. This is the scale that we shall have in mind when saying "mesoscopic", though for the most part the effects that we shall be discussing can be squeezed into a narrower length-scale span of single nanometers to several tens thereof.

\section{Concept of size-dependent mesoscopic relaxation and its effect on nanostructure growth and elec- tronic/magnetic properties}

Nowadays, with the solid basis of theoretical and experimental works briefly mentioned in the introduction, one has a set of expectations, as to what geometric distortions a nanostructure epitaxially adsorbed or grown on a surface might undergo. A benchmark for a theory incorporating those expectations is its ability to explain, if not to predict a priori, stress and strain arising in the system, as well as their impact on the other physical properties of the system on the mesoscopic scale. Before we proceed to defining such a theory, let us engage in a short Gedankenexperiment and imagine a nanostructure of material "A" being grown on a surface of crystal "B" (Fig. 2(a)). For the sake of simplicity, let us say that the native lattice constant of A is smaller than that of B. First and foremost, we would expect, that the natural misfit and reduced coordination at the edges of the nanostructure would result in a contraction of its interatomic bond lengths. The latter does not have to be limited to the edges of the structure, but could reach far into its interior (Fig. 2(b)). The deformations of the structure are not limited to in-plane contraction or expansion but are three-dimensional, i.e. strong vertical relaxations of the ad-structure can also be expected (Fig. 2(c)). The substrate also does not remain unaffected and might be strongly corrugated by the compressive and tensile stresses exerted by the ad-structure (Fig. 2(d)). The effects, that we have just listed for the case of hetero-epitaxy are well understood and captured to a larger or lesser degree even by simple models, such as the continuum elasticity framework, introduced by $\mathrm{Hu}$ and coworkers. 39] Those theories, however, relying on the natural misfit of the two materials alone, start to result in quantitative discrepancies when the size of the ad-structure shrinks to the meso-scale (as shall be discussed in more details in the following) and utterly fail in the description of homo-epitaxial growth and adsorption. Yet nowadays it is well known (see Sec. 3.2) that homo-epitaxial structures are subject to much the same relaxation patterns as were just listed for the case of heteroepitaxy. As shall be shown in the following, strain fields encountered in homo-epitaxy span both the nanostructure and the substrate underneath and around the latter and can have extremely intricate morphologies (Fig. 2(e)). The concept of mesoscopic misfit was devised to overcome the shortcomings of continuum elasticity theory. It can capture the above mentioned effects and accurately predict various other ones, like, for example, the behavior of embedded ad-structures, which are also subject to mesoscopic relaxations, both in the body of the structure and in the host surface, in-plane as well as out-of-plane of the latter (Fig. 2(f)).

As has been just mentioned, the classical way of predicting and understand growth of ad-structures on various substrates has, for a long time, been based on the notion of 
(a)

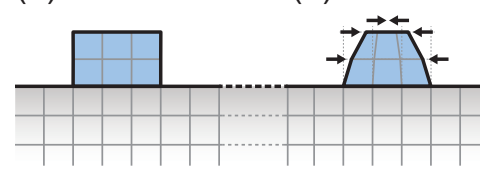

(c)

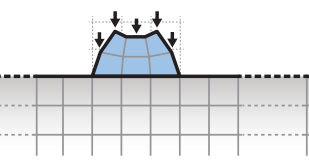

(d)

(e)

Figure 2: A schematic illustration of the relaxations hetero- and homo-epitaxial systems. Atoms of ad-structures (a) on surfaces have a reduced coordination, which as a rule results, according to the Pauling's principle, in a contraction of interatomic distances not only at the edge, but reaching far into the interior of the ad-structure (b). Moreover, not only in-plane interatomic distances are affected, but also strong vertical relaxations of the ad-structure can be observed (c). The substrate also does not remain unaffected and is either depressed of supinated underneath the structure $(\mathrm{d})$. While for hetero-epitaxial systems many of the above-mentioned effects are very pronounced due to the intrinsic/natural mismatch, homo-epitaxial systems are also to mesoscopic relaxations (e) which result in complex strain fields in the ad-structure and the substrate underneath. Even embedded ad-structures are subject to mesoscopic relaxations, causing strong relaxations in the system both in- and out-of-plane.

natural misfit - the difference in the intrinsic equilibrium bond lengths of surface and adsorbate materials. Such mismatch at the "film-substrate" interface leads to formation of far-reaching strain fields. The system has the possibility to relieve the stress either plastically (through defects formation - dislocations, grain boundaries, lattice distortions and so on) or elastically (through atomic relaxations). In conventional continuum elasticity theory, which has for a long time been the de facto principal theory for understanding interface distortions, strain relief is described on the basis of the macroscopic or natural lattice mismatch $m_{0}=\left(a_{a d s}-a_{s}\right) / a_{a d s}, a_{a d s}$ and $a_{s}$ being the equilibrium bond lengths of the adsorbate and surface materials. However, if the deposited system is of a "mesoscopic" size of several hundred atoms, its intrinsic bond lengths are far from the bond lengths of both respective bulk crystals. In this case the local modification of the electronic structure and bond properties of the system can lead to strong positional dependence of the interatomic bond lengths on the atomic length scales, resulting in what is nowadays called "mesosopic mismatch" - a mismatch that occurs in finite structures, varies on a sub-nanometer scale (depending not only on the natural misfit, but on the local environment of particular atoms) and often results in complex 3D strain fields in both the ad-structure and the substrate.

In this chapter we shall demonstrate several scenarios of strain relief in mesoscopic islands in metal hetero- and homo-epitaxy, which shall leave no doubt that the mesoscopic mismatch, rather than the macroscopic one, is the proper way of describing strains and constitutes the driving force for strain relieving effects at the mesoscale. After providing ample evidence that mesoscopic misfit leads to profound strain relief (geometric distortion) patterns in ad-structures and supporting substrates in both heteroand homo-epitaxial systems we shall proceed to the discussion of possible implications of such misfit. We shall start by tracing the effect of the latter on atomic kinetics and nucleation, which in turn define growth patterns and morphology of epitaxial ad-structures. From there it is a small step to tracing the cumulative effect of strain relaxation and morphology on the electronic and magnetic properties of the system, which we shall indeed do in the last few sections of the present chapter.

\subsection{Hetero-epitaxial metal growth}

Let us start by showing that even in the case of heteroepitaxy, which was thought to be well understood in the confines of the continuum elasticity model, some effects are impossible to comprehend without considering the mismatch to be an inherently local, mesoscopic property of the system. As has been mentioned in Sec. 2, several stress measurements showed that native lattice mismatch arguments are often inappropriate for understanding the strain relief in the early stages of the hetero-epitaxial growth. 36, 40] Referring the reader for more details to Sec. 6. here we shall only mention that while tensile stress should be expected in such systems as Fe, $\mathrm{Co}, \mathrm{Ni}$ and $\mathrm{Cu}$ on W(110) in the sub-monolayer growth regime, compressive stress was found experimentally, 40, 73 the strength and sign of which not even the advanced modifications of continuum elasticity theory could explain, let alone predict. A giant compressive stress for the first few monolayers of $\mathrm{Ag}$ on $\mathrm{Pt}(111)$ was reported to be far beyond the stress expected from the lattice mismatch between $\mathrm{Ag}$ and Pt. 74] This is one of many examples of the questionable relevance of the continuum elasticity approach for early stages of epitaxial growth.

For a vivid demonstration of the advantages of the mesoscopic misfit concept we turn our attention to systems with small macroscopic lattice mismatch. Here especially, as we shall shortly see, the sub-monolayer growth in the initial stages of metal film hetero-epitaxy is influenced by the mesoscopic strain relaxation. In this section we focus largely on Co nano-islands grown atop $\mathrm{Cu}$ substrates. Several reasons can be named for taking this particular system as a prototype. One is that growth of $\mathrm{Co}$ on $\mathrm{Cu}(001)$ has historically been of great general interest in the early years of magnetoelectronics bloom, since the quality of the $\mathrm{Co} / \mathrm{Cu}$ interface has a strong influence on such fundamental properties of nano-scale systems and devices as giant magnetoresistance, magnetic anisotropy and oscillatory exchange coupling. [75, 76] The second reason, as already been hinted, is the smallness of the macroscopic mismatch between cobalt and copper $(2 \%)$. This would, from the naive point of view, suggest a small tensile strain in Co nanostructures on, e.g., $\mathrm{Cu}(001)$. The more unexpected was the occurrence of in-plane lattice spac- 
ing oscillations (IPLSOs) in that system, which were observed by means of reflection high-energy electron diffraction (RHEED) during Co epitaxy on $\mathrm{Cu}(001)$ in pseudomorphic regime. 30 No macroscopic mismatch argument could account for those observations. It was shown that for $\mathrm{Co} / \mathrm{Cu}(001)$ system the growing Co monolayers are periodically contracted for half-integer coverages. Monolayer stress oscillations, discussed in Sec. 6.2 corroborates this finding. 77] Later on, this result was additionally verified for two-dimensional hetero-epitaxial growth on (001) surfaces of cubic metals using the same RHEED technique for numerous hetero-epitaxial systems with positive (V/Fe, $\mathrm{Mn} / \mathrm{Fe}$ ), negative $(\mathrm{Ni} / \mathrm{Fe}, \mathrm{Ni} / \mathrm{V}, \mathrm{Co} / \mathrm{Cu}$ ) or close-to-zero $(\mathrm{Fe} / \mathrm{Cu}, \mathrm{Co} / \mathrm{Ni})$ misfits. 42] A similar effect of in-plane lattice spacing oscillatory behavior was observed on $\mathrm{Mn}$, $\mathrm{V}$ and $\mathrm{Ni}$ on $\mathrm{Fe}(001), \mathrm{Ni}$ on $\mathrm{V}(001)$, and confirmed once again the results of $\mathrm{Co} / \mathrm{Cu}(001)$ reported earlier by Fassbender et al. 30

On par with experimental observations, several ab initio studies, for example of Pentcheva and coworkers, 78, have shown that for very thin $\mathrm{Co}$ film on $\mathrm{Cu}(001)$ the comparison of bulk lattice constants of $\mathrm{Co}$ and $\mathrm{Cu}$ is inappropriate as a measure of the stress in Co layers. [78] As theoretical predictions go, a few years prior to the studies of Pentcheva et al. the forerunner of the mesoscopic misfit concept evolved from the classical elasticity theory. Kern, Müller and others 41] have advocated the use of the so-called "active misfit", a correction to the natural misfit accounting for the reduced coordination at the nanostructure's edges. The principal idea was that atoms at step edges shall attempt to undergo relaxations to increase their binding energies by maximizing the nearest neighbor number. 41] The atoms were predicted to relax towards the center of the nanostructure (cf. Fig. 2(b)) and assume new equilibrium positions with shorter bonds than that of the adsorbate material bulk. It were arguably those extensive theoretical studies that marked the dawn of the mesoscopic misfit concept as a crucial part of describing all epitaxial phases barring the infinite ones.

We shall address "active misfit" in a more detailed fashion in Sec. 3.3 . Here we just note, that active misfit, defining an effective mismatch as a size-dependent characteristic of an ad-structure, has been an intermediate step between the continuum-elastic natural misfit and the contemporary meso- and microscopic misfit as a truly local property varying even within a single nanostructure.

To give a few quantitative examples of mesoscopic misfit and strain relaxations in epitaxial nanostructures, here we show the island-size dependence of the average bond length of two-dimensional compact $\mathrm{Co}$ islands on $\mathrm{Cu}(001)$ (see Fig. 3 36 determined from fully relaxed geometries of the clusters (obtained in quasi-ab-initio molecular dynamics simulations based on the tight-binding approximation for many body potentials). 38. It can be observed that average bonds in the islands are shorter than the bond length of both $f c c$ Co and $\mathrm{Cu}$ bulk materials. This finding is completely contra-intuitive from the continuous

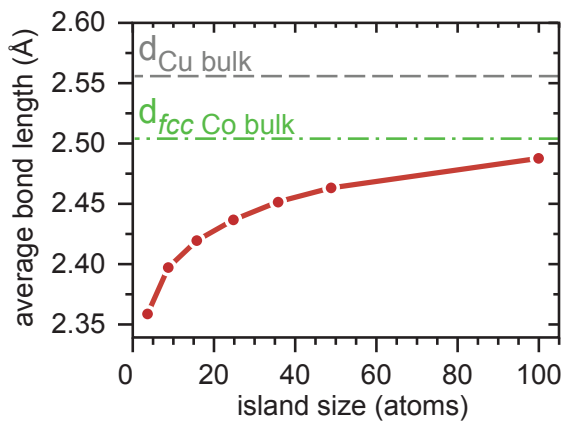

Figure 3: The average Co-Co bond length in the two-dimensional compact $\mathrm{Co}$ islands on $\mathrm{Cu}(001)$. Figure adapted with permission from Ref. 36 .

elasticity point of view, since such contraction is due to the strong relaxation of edge atoms and partially even the atoms of the island interior. With increasing size of the cluster, the effect of edge atoms becomes less pronounced as the average bond length increases. Yet even in a Co island of 100 atoms $\left(\mathrm{Co}^{100}\right)$ the average bond length is noticeably smaller than that in $f c c$ Co bulk. In Sec. 3.7 we shall especially address the question of the truely local dependence of the misfit across the island, but already the above results are a strong hint that strain relaxations in small ad-structures should lead not only to pronounced structural changes in the shape of the structure itself, but are bound, through induced stress, also affect the shape of the underlying and surrounding substrate areas.

As an evidence supporting this "guess", Fig. 4 shows the atomic displacements in a $\mathrm{Co}^{36}$ island and in the surrounding substrate along the $\langle 110\rangle$ direction (Figs. 4(ac)). 36 Here it should be noted that apart from pronounced in-plane relaxations in the system, a strong outof-plane displacement can be observed. The substrate atoms under the island are pushed down, while substrate atoms at the outer edge of the Co island are pushed up. The average strain (determined from bond length analysis) in the substrate layer under the cluster in-plane of the surface is negative, i.e. the substrate atoms are under compressive strain. However, it has to be noted that displacement fields under and near Co islands found in the above study are very similar to the results obtained for individual surface steps using elastic theory. While often underestimating the effects, the latter succeeded in predicting strain relaxations near steps on $\mathrm{Ni}$ and Au surfaces. 79] Those studies reported finding atomic displacement fields near surface steps exhibiting a "nontrivial behavior": existence of a plane across which the out-of-plane atomic displacements change sign, i.e. the surface is corrugated in a fashion of a wave spreading around the ad-structure. A similarly strong inhomogeneous strain distribution in the surface layer was reported during extended analysis of Co triangular islands on $\mathrm{Cu}(111)$. 43. Sizable compressive strains at $\mathrm{Cu}$ atoms below $\mathrm{Co}$ islands and a tensile strain at the boundary thereof constituted another interesting theoretical prediction. 


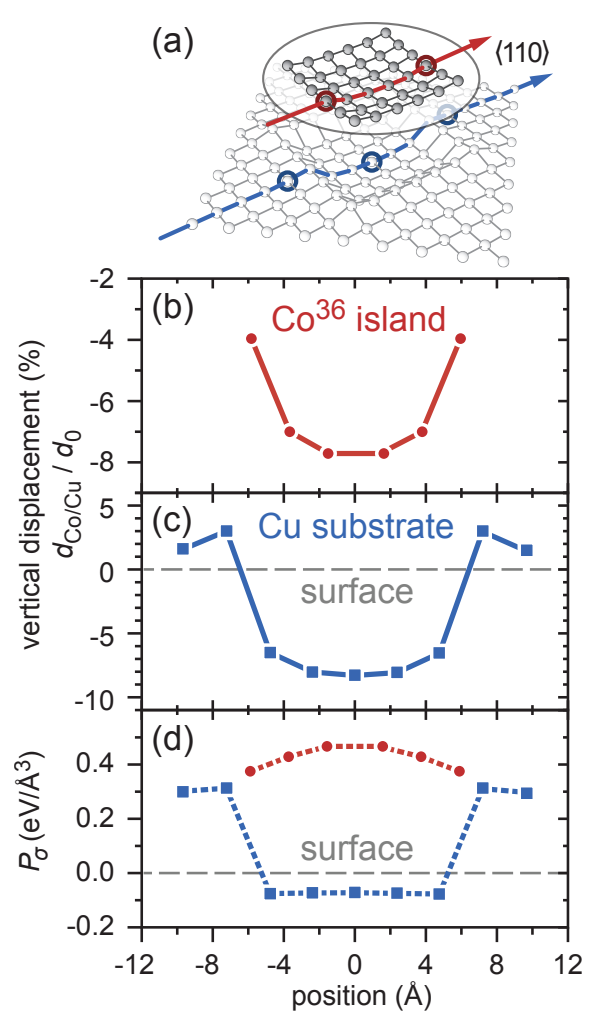

Figure 4: (a) Sketch of the system (a $\mathrm{Co}^{36}$ square island on $\mathrm{Cu}(001)$ surface) and a 3D representation of relaxations. (b) Vertical displacement of Co atoms of the island and (c) $\mathrm{Cu}$ atoms of the topmost substrate layer along the $\langle 110\rangle$ direction. The interlayer distance is $d_{0}=1.8075 \AA$ and the lattice constant of $\mathrm{Cu}$ is $a_{0}=3.615 \AA$. (d) Hydrostatic stress for the same island and substrate layer. Figures adapted with permission from Ref. 36
The effects described above are, most certainly, not only particular to systems with square lattices (as those mentioned up to now). Triangular Co islands on $\mathrm{Cu}(111)$ were found to exhibit deformations comparable to those of square-shaped islands on $\mathrm{Cu}(001)$, strongly supporting the general applicability of the concept of mesoscopic misfit to the structures formed at early stages of epitaxial thin film growth. 36, 40,

Finally, it is important to note that theoretical examinations of strained $\mathrm{Co} / \mathrm{Cu}$ systems do not simply reveal strongly inhomogeneous deformations in the system. They give a unique access to the local picture of stress and strain, something that to-date experimental techniques cannot yet provide. As an example, Fig. 4(d) shows the atomically resolved hydrostatic stress $P_{\sigma}=\operatorname{Tr}\left(\sigma_{\alpha \beta}\right)$ in a $\mathrm{Co}^{36}$ island and in the surface layer of $\mathrm{Cu}$. From the theoretical perspective, the hydrostatic stress calculations is simply based on the determination of atomic level stress components. 80 ]

One can observe in Fig. 4(d) the spatially varying nature of the stress on the $\mathrm{Cu}$ atomic layer. At the island edge, the stress is highly tensile, while the substrate layer under the island is seen to exhibit compressive hydrostatic stress. The tensile hydrostatic stress at the cluster center is different from that at the edge, which is another hint that sometimes it might even be necessary to treat the misfit microscopically, rather than on the meso-, let alone on the macro-scale. In the following sections we shall discuss how the inhomogeneous stress distribution in the islands influences the motion of atoms on the islands themselves, as well as on the substrate in the vicinity of the islands.

\subsection{Homo-epitaxial metal growth}

Even stronger indication of the importance of considering misfit as a mesoscopic quantity is the case of homoepitaxy. In that case, according to the classical rules of elasticity theory, no difference between deposited material and substrate exists and no effects are expected to arise due to the absence of natural misfit. The principal drawback of this approach is obvious and has been recognized long ago. [36, 40, 41 In reality, not only mesoscopic islands, but also their macroscopic "peers" have, due to reduced effective coordination, intrinsic bond lengths which can be different from the bulk bond lengths of corresponding materials. Moreover, as it has been hinted in the previous section, this mismatch is not constant across the island, but is strongly position dependent. The relaxation of both edge and interior atoms in mesoscopic islands can be the dominating process. Similarly to the case of hetero-epitaxial systems, these atoms relax towards the center of the nanostructure in order to increase their binding energies.

It is a well established notion in crystallography of freestanding clusters, that small metallic crystallites exhibit substantially smaller lattice constants as compared to their macroscopic equivalents. 81] It took time for this notion to establish itself in the world of epitaxy. First conclusive hints came, as in the case of hetero-epitaxy, from then 


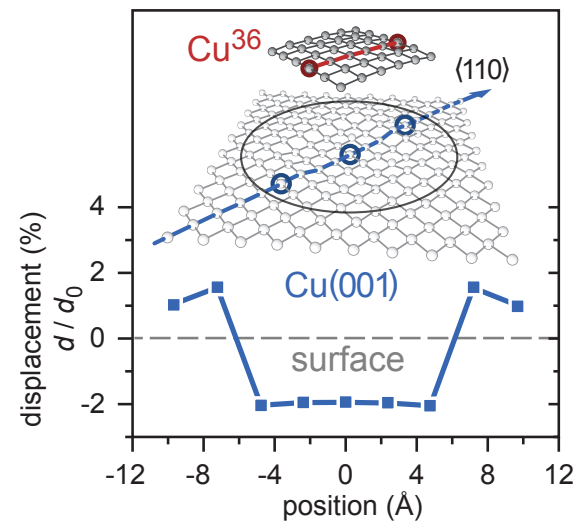

Figure 5: Relative vertical displacements $\left(\mathrm{d}-\mathrm{d}_{0}\right) / \mathrm{d}_{0}$ of the atoms in the topmost layer of $\mathrm{Cu}(001)$ under $\mathrm{Cu}^{36}$ island calculated across the $\langle 110\rangle$ direction. Here $\mathrm{d}$ and $\mathrm{d}_{0}$ are the calculated and equilibrium interlayer distances. Figure adapted with permission from Ref. 43

unexpected in-plane lattice spacing oscillations (IPLSOs) during native epitaxial growth of $\mathrm{Cu}$ on $\mathrm{Cu}(001)$ single crystal substrate in the pseudomorphic growth regime. 30. The authors found periodic expansions for half-integer coverages, which was beyond conventional description based on macroscopic mismatch arguments.

Similarly strong effects in IPLSOs have been observed by RHEED during two-dimensional homo-epitaxial growth of $\mathrm{V}(001), \mathrm{Fe}(001)$ and $\mathrm{Nb}(001)$ surfaces and was first considered to be due to surface reconstructions and/or contamination. 42] Indeed, this study on IPLSOs for these homo-epitaxial systems demonstrated that elastic strain relaxations at the edges of two-dimensional islands can have the same amplitude range, as in hetero-epitaxial systems, leaving beyond doubt the need for a more complete model of epitaxial misfit.

Here again, to give the reader a truly local and quantitative picture of the strain relaxations in a homo-epitaxial system we resort to an example from theory. Accurate atomic scale simulations (identical to this discussed in the previous section) were performed for square $\mathrm{Cu}$ islands on a $\mathrm{Cu}(001)$ substrate. It was confirmed that small $\mathrm{Cu}$ islands have a different bond length compared to $\mathrm{Cu}$ bulk. Thus inhomogeneous strain distribution can be found both in the islands themselves and in the $\mathrm{Cu}$ substrate. Atomic rearrangements in a $\mathrm{Cu}$ substrate induced by a $\mathrm{Cu}^{36}$ island are shown in Fig. 5, revealing unmistakable structural changes in the shape of both the island and the substrate, albeit smaller than in the hetero-epitaxial case of $\mathrm{Co} / \mathrm{Cu}(001)$.

As a further example, a similar scenario of elastic strain relaxations was reported to affect growth and morphology of hexagonal $\mathrm{Cu}$ islands on $\mathrm{Cu}(111)$, 37] showing again that the symmetry of the system does not alleviate the effect of mesoscopic misfit. Here again strong inhomogeneous strain distribution in islands and substrate can be observed, as shown in Figs. 6(a) and 6(b), displaying atomic displacements in a $\mathrm{Cu}^{19}$ hexagonal island on

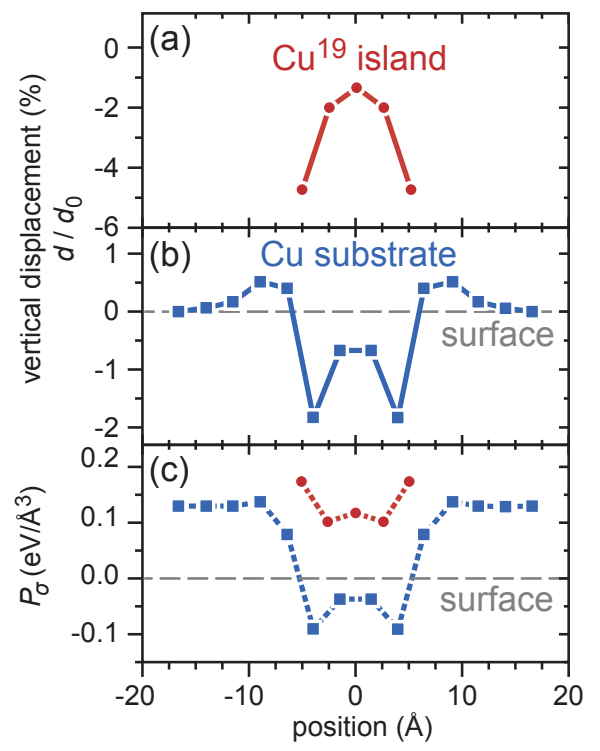

Figure 6: Vertical displacements of $\mathrm{Cu}$ atoms in the $\mathrm{Cu}^{19}$ island (a) and the surface atoms in the topmost substrate $\mathrm{Cu}$ layer underneath the island (b) along the $\langle 110\rangle$ direction. Interlayer distance $d_{0}=$ $1.8075 \AA$ and lattice constant $a_{0}=3.615 \AA$. (c) Hydrostatic stress induced in $\mathrm{Cu}^{19}$ island and the substrate underneath by mesoscopic misfit. Figure adapted with permission from Ref. 37

$\mathrm{Cu}(111)$ along the $\langle 110\rangle$ direction. The substrate atoms under the island are pushed down, and the island assumes a convex shape. The surface is locally distorted and a sizable and strongly inhomogeneous displacement pattern is observed therein. The residual hydrostatic stress in the $\mathrm{Cu}^{19}$ island and the substrate underneath is shown in Fig. 6(c). One can see that also in the case of homoepitaxy mesoscopic misfit leads to an inhomogeneous stress distributions in the sample. At the island edge, the stress in the substrate is tensile (positive), while the substrate layer under the island is seen to exhibit compressive (negative) stress. The tensile hydrostatic stress at the cluster center is different from that at the edge, once again stressing the locality of the phenomenon.

Thus, similar to hetero-epitaxy 36] mesoscopic islands in homo-epitaxial growth introduce stress in the surface region that can vary on an atomic scale. At the time of publication, these results were challenging the conventional view of the lattice coherence between islands and the substrate in homo-epitaxy, promoting the necessity to turn from classical continuum elasticity view of epitaxy to a more complete mesoscopic misfit picture.

\subsection{Size-dependent mesoscopic misfit}

Now let us spend a few lines to introduce the concept of size-dependence mesoscopic misfit. Although, as already mentioned, the truly complete picture of the misfitinduced phenomena is the one, where misfit is considered in an atomically-resolved fashion, experimental findings obtained from stress measurements [40, 73] or from electron diffraction [30] during sub-monolayer growth in 


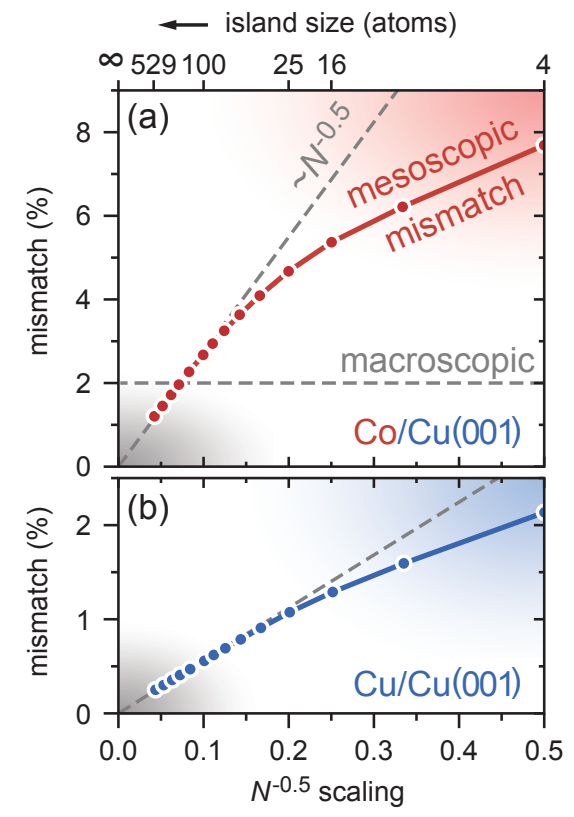

Figure 7: Evolution of the average mesoscopic mismatch $m=\left(r_{b}\right.$ $\left.r_{0}\right) / r_{0}$ for $\mathrm{Co}(\mathrm{a})$ and $\mathrm{Cu}(\mathrm{b})$ square islands as a function of island size $N . r_{b}$ is the average bond length in $\mathrm{Co} / \mathrm{Cu}$ islands, $r_{0}=2.556 \AA$ is the bond length of $\mathrm{Cu}$ bulk. Note that the size $N$ of the islands increases from right to left. Figure adapted with permission from Ref. 49

metallic systems can often be understood within the framework of the classical theory of elasticity, or an enhanced version thereof, where one corrects the value of natural misfit to account for the finite size of the structure. The resulting misfit, introduced by Kern and Müller 41] was termed "active misfit". The authors stressed the real importance of elastic strain relaxations, arising due to sizeeffects at the free edges of islands, taking them into consideration when dealing with growth behavior within submonolayer-coverage regimes. 41] The "active misfit" is supposed to be an averaged characteristic of a nanostructure, depending solely on its size.

Let us explore the boundaries of applicability of this theory by benchmarking it against quasi-ab-initio molecular dynamics calculations (the same method as has been used to obtain the results presented in preceeding sections). In Fig. 7(a) the average mesoscopic strain in square Co islands on $\mathrm{Cu}(001)$ is shown as a function of the size of the latter. [49] The mesoscopic misfit $m$ was determined by evaluating atomic bond lengths in Co islands as $m=\left(r_{b}-r_{0}\right) / r_{0}\left(r_{b}\right.$ and $r_{0}$ being the average bond length in Co islands and $\mathrm{Cu}$ bulk, respectively). Note that here no assumption was made about the analytical dependence of the average misfit on island size (as is the case in the original concept of the size-dependent misfit), but rather a straightforward averaging of real bond lengths is carried out, another courtesy of the atomistic theoretical approach.

It can be seen, that mesoscopic misfit between small $\mathrm{Co}$ islands and the $\mathrm{Cu}$ substrate is considerably larger than the macroscopic misfit $m_{0}$, defined as $m_{0}=\left(a_{\mathrm{Co}}-\right.$ $\left.a_{\mathrm{Cu}}\right) / a_{\mathrm{Co}}\left(a_{\mathrm{Cu}}\right.$ and $a_{\mathrm{Co}}$ being the lattice constants of $\mathrm{Cu}$ and Co bulk crystals) which is rather small $(\sim 2 \%)$. Only for Co islands comprised of more than 200 atoms the local elastic strain can be approximately described by the macroscopic misfit.

If we consider that the relaxation edges is the dominating process, 30, 49] we should assume that the misfit scales with the relative fraction of atoms belonging to the edge. For a monolayer-thick island with $N$ atoms this would mean a scaling law of $N^{-0.5}$ (gray dashed line in Fig. 7(a)). 49 And indeed, for Co islands larger than 60 atoms the misfit does follow the $N^{-0.5}$ rule fairly closely. For Co islands smaller than 50-60 atoms, however, the deviation from the rule becomes more and more apparent, as the edge fraction starts to dominate and becomes extremely island-shape-dependent. Thus, for a certain island-size range, given the assumption that the shape of the islands is fairly regular (f.e. in fractal islands the perimeter scaling shall not be subject to the $N^{-0.5}$ rule), the true mesoscopic misfit can be approximated by the "size-dependent" one, if one is content with capturing in the model the average bond length of a nanostructure. Yet for a truly universal description, the use of the mesoscopic misfit concept is indispensable.

Here, as well, the findings and conclusions acquired from hetero-epitaxial system analysis can be transferred to the case of homo-epitaxy (see Fig. 7(b) for an example of square $\mathrm{Cu}$ islands in $\mathrm{Cu}(001)$ surface). 37, 43] Here, no approximation with macroscopic misfit is possible, the latter being exactly zero. Yet for large islands $(\geqslant 25-50$ atoms) the $N^{-0.5}$ approximation can be applied to interpret experimental observations. For smaller islands, the same breakdown of the $N^{-0.5}$ law can be observed as in the case of hetero-epitaxy.

To give another example of the same physics, structure and growth of $\mathrm{Fe}$ deposits on reconstructed $\mathrm{Au}(111)$ have been investigated experimentally by grazing incidence $\mathrm{x}$ ray diffraction (GIXD). As a consequence of the epitaxial growth, Fe-Fe bond lengths were found to be expanded to the underneath lattice spacing (up to $12 \%$ compared to the high-temperature $f c c$ Fe phase) and the average interatomic distances of the growing islands and films was found to vary as a function of Fe coverage (and thus Fe island size). 82

To draw a bottom-line for this section, we shall again point out, that the concept of "active", or "size dependent" misfit dealt with an effective mismatch as an averaged characteristic of an ad-structure as a whole, thus capturing some of the experimentally observed effects in nanostructures exceeding a certain limit in size. It was an important intermediate step from the level of continuum elasticity towards the contemporary understanding of the mesoscopic misfit as a truly microscopic quantity, defined on the meso- and microscopic level and varying, as shall be discussed in the following, even within a single nanostructure. 

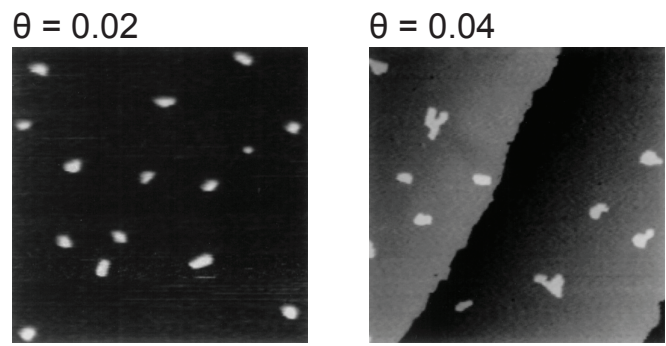

$\theta=0.15$

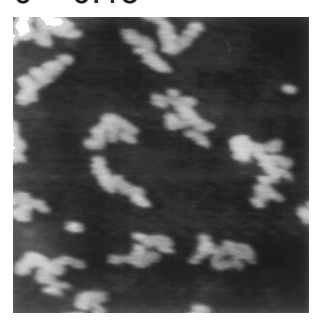

$\theta=0.32$

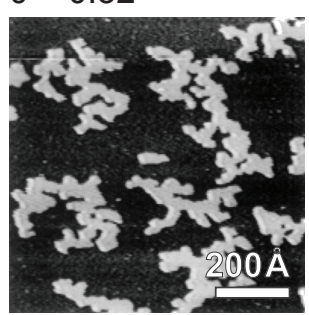

Figure 8: Sub-monolayer growth of copper islands on $\mathrm{Ni}(100)$ characterized by a transition from compact to ramified island shapes with increasing island size. The islands are grown at a substrate temperature of $345 \mathrm{~K}$ and a growth rate of $1.5 \times 10^{-3} \mathrm{ML} / \mathrm{s}$. Figure adapted with permission from Ref. 35

\subsection{Size-dependent shape transitions in mesoscopic islands}

Having outlined the general relaxation patterns of nanoislands induced by mesoscopic misfit, we now come to the first example of how the misfit can affect the macro- and meso-scopically observable properties of the system. One important ramification of the lattice misfit between a deposit and a substrate is the so called "strain-induced shape transition" in nano-islands, which was predicted by Tersoff and Tromp. 28] It was shown in low energy electron microcopy studies of $\mathrm{Ag}$ growth on $\mathrm{Si}(001)$, that below a critical size silver islands have a compact symmetric shape, but at larger sizes they adopt an elongated shape due to favorable elastic relaxations of the island stress. Also Mundschau et al. 83] and Rousset et al. 84, have observed elongated island formation during $\mathrm{Au}$ growth on $\mathrm{Mo}(111)$ and $\mathrm{Ag}(110)$ respectively. It was shown in these cases, that when the stress is anisotropic the island should align itself perpendicular to the direction of maximum stress. Later on, Müller et al. 35] demonstrated that strain relaxations in islands can lead to the growth of ramified structures even at interfaces with square symmetry. Their scanning tunneling microscopy (STM) study has shown that $\mathrm{Cu}$ islands on $\mathrm{Ni}(100)$, as they grow in size at sub-monolayer coverages, undergo a shape transition from compact to ramified islands at a critical island size (see Fig. 8). The critical island size has been assessed by the authors to be $\sim 480$ atoms for $\mathrm{Cu} / \mathrm{Ni}(100)$, based on the analysis of the island border dimensionality. 35. The ramified shape of larger copper islands was ascribed to anisotropic sizedependent strain relief at island edges. Moreover, earlier RHEED results indicated strong anisotropic behavior of in-plane lattice spacing oscillations, obtained for twodimensional islands grown on square lattices in different metallic hetero- and homo-epitaxial systems. These were ascribed to the strong shape anisotropy of the islands leading to equally anisotropic strain relaxations patterns. 42] These studies explicitly demonstrated that a delicate balance between the strain energy (due to lattice misfit) and the bond energy within the island determines the shape of a growing structure.

Acknowledging the pronounced size dependence of the misfit-induced energy, one can expect that the latter can play a significant role in both growth patterns and final morphology of the epitaxial system. For example, island coalescence will dominate for larger coverages since it reduces the strain relaxations in the islands and in the surface layer. The competition between $\mathrm{Co}-\mathrm{Co}$ and $\mathrm{Co}-\mathrm{Cu}$ interactions is also a driving force for the shape transitions in the clusters and the substrate. [36, 42, Calculations performed by Stepanyuk and coworkers 36. showed that $\mathrm{Co}-\mathrm{Co}$ interaction is stronger than $\mathrm{Co}-\mathrm{Cu}$ and $\mathrm{Cu}-\mathrm{Cu}$ interactions, and, consequently, the strain relaxations of $\mathrm{Co}-\mathrm{Co}$ interatomic distances in the Co cluster are stronger. The reported changes in the cluster shapes and the substrate were deemed likely to be of general importance and were prophesied to have a profound effect on island growth.

It is only fair, however, to mention here that the step bending and island shape transitions have been also predicted before using the continuum-like theory of elasticity. 85, 86. Li et al. [86] described theoretically the effect of elastic strain on the equilibrium shape of a twodimensional island. It was shown that in homo-epitaxial system the equilibrium shape of the islands under stress is derived from both island step free energies and strain energies. The difference in strain energy for different island sizes is predicted by that theory to lead to a complex evolution of island shape with increasing island size. The latter is expected to depend on the relative strengths of step and strain energies and on the anisotropy of the former. It was stressed that such behavior can be observed even in homoepitaxial islands if a surface stress anisotropy induced by the substrate is present. Thus the shape evolution of the islands discussed in Sec. 3.2 for the hetero-epitaxial is a more general phenomenon. Both for homo- and heteroepitaxy, the strain makes the island shape size-dependent.

Atomic scale simulations of Stepanyuk et al. [43] demonstrated the validity of this statement, showing that homoepitaxy at the meso-scale can be accompanied by shape transitions in square $\mathrm{Cu}$ islands on $\mathrm{Cu}(001)$. In that case, however, the "shape transition" reported by the authors should be understood as vertical deformation of the island, rather than the change of island boundary shape or dimensionality. Specifically, Stepanyuk et al. have shown that a $\mathrm{Cu}$ island assumes a "tent-like" shape evolving as the islands grow in size. The edge atoms thereby are the lowest. The $\mathrm{Cu}$ surface atoms under the $\mathrm{Cu}$ island are pushed down into the substrate, while atoms at the outer edge of the $\mathrm{Cu}$ island are pushed up. 


\subsection{Adsorption, diffusion on and near mesoscopic islands}

If we step back and take another look at the findings summarized in the past sections, it becomes apparent that mesoscopic misfit is both strongly dependent on the nanostructure size (Secs. 3.1 3.3) and in turn itself defines the morphology and dynamics of epitaxial growth (Sec. 3.4). To better understand this self-consistent interrelation between size, shape and misfit let us consider how the basic processes of atomic adsorption and diffusion depend on the presence of stress and strain in the substrate and the nanostructures thereon. Once this interrelation is clear, we shall generalize the attained conclusions by pointing out various other implications of mesoscopic misfit for nanostructure growth and morphology.

Let us start by considering the growth modes from the point of view of mesoscopic effects. We have shortly outlined the three currently distinguished growth modes in the introductory section $(2 \mathrm{D}, 3 \mathrm{D}$ and $\mathrm{SK})$. On the atomistic level, growth is determined by static and dynamicsrelated parameters, the former being the adsorption energies of adatoms in different environments, defining the ground state of the nanostructure (which may or may not be reachable) and the latter being the diffusion barriers, which determine the system's thermal and temporal stability and its evolution in general. 87. There are a few principal barriers that shall be important to us in the following. First of all, it is the hopping barrier, which determines the ease with which an adatom can travel across the surface or on top of a nanostructure. We shall show in the following that this barrier is rather sensitive to the strain in the substrate and the lattice spacing thereof. The second important barrier governs the interlayer mass transport. If an adatom has to traverse the edge of a nanostructure or a step in the surface it has two possibilities, either to jump over the edge (Ehrlich-Schwoebel barrier) or to push one of the edge atoms aside, taking its place (exchange barrier). Yet even before a traveling adatom can attempt to scale the edge of a surface step it has to approach the latter, which is sometimes hindered by a repulsive barrier existing in the vicinity of nanostructure or terrace boundaries. This barrier, which has been known to surface physicists for several decades, has only recently been realized to be a manifestation of mesoscopic strain relaxation. Examples of the dependence of all those barriers on local strain fields in the substrate and the step/nanostructure shall be shown presently. For the formation of embedded structures another barrier, the substitutional one, is the control parameter. Let us start off by considering the effect of strain on each of the above-mentioned processes.

Phenomenologically, two-dimensional growth (2D) is expected in an epitaxial system if adatoms, diffusing on a surface, can traverse the edges of nanostructures and steps. If deposited atoms are not free to "hop" on and off the islands, three-dimensional (3D) growth shall usually be promoted. In general, the delicate balance between the interlayer transport, diffusion, deposition rate, and the size

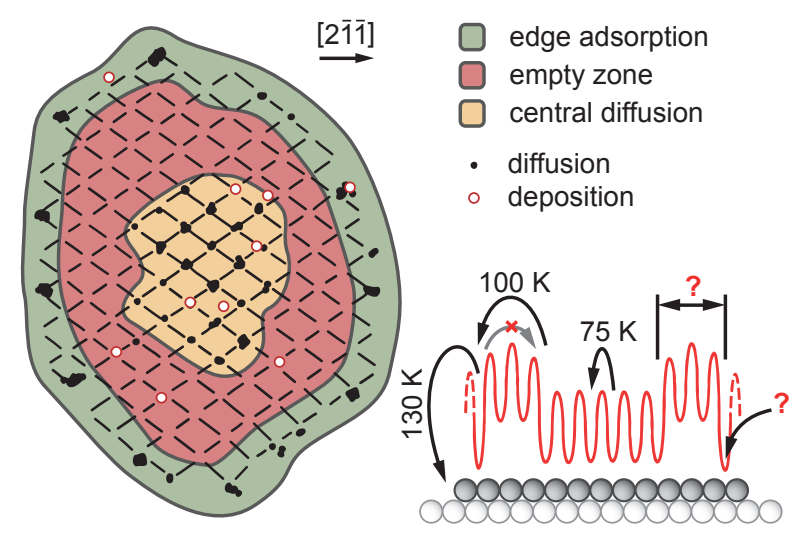

Figure 9: Map of sites occupied by Pt atoms on the Pt(111) cluster of $\sim 145$ atoms. Filled circles mark the location of the atom after diffusion. Red circles identify atoms after deposition at $\sim 20 \mathrm{~K}$. Only one atom is present on the cluster at any one time during mapping. The empty zone separating the central region from the cluster edge can be populated by condensing atoms. The schematics in the bottom right part of the figure shows a sketch of the adsorption potential on the island and the temperature at which particular transport processes become operational. Figure adapted with permission from Ref. 88 .

and shape of islands determines, in a self-consistent fashion, the growth mode.

To start with the simplest process of atomic diffusion, we note that direct observation of adatom movement on islands was first undertaken in field ion microscope (FIM) experiments by G. Ehrlich and coworkers (Wang, Golzhäuser et al). 88 90 These experiments demonstrated that motion of adatoms on top of islands differs from that on a flat surface. An empty zone separating the central region from the cluster edge was observed for Ir diffusion on $\operatorname{Ir}(111) 90$ and for Pt diffusion on Pt(111) (see Fig. 9). 88. It was suggested that the empty zones come about as a consequence of stress induced geometric relaxations causing an area of unfavorable adsorption to separate the center and the rim of the islands, so that individual atoms have to overcome an energy barrier just to cross this empty zone and reach the cluster edges.

The "mystery" was later clarified by Brovko and coworkers 91 who have shown that the effect observed by Wang and Ehrlich [88] is the consequence of a delicate balance between mesoscopic relaxations in the system, reduced coordination numbers at the edges of clusters and, most importantly, relativistic effects. Fig. 10 shows the adsorption energy profile for a single $\mathrm{Pt}$ atom on top of a $\mathrm{Pt}$ island on Pt(111) surface for relaxed and unrelaxed geometries. 91. It is apparent, that while the relaxation play an important role in defining the diffusion characteristics, they alone cannot account for the observations of Wang and Ehrlich. They do, however, play a indispensable role in the complex picture.

Another approach to understanding the interplay of misfit and diffusion was taken by Brune et al. 93, who conducted STM measurements of Ag adatoms on a $\mathrm{Ag}(111)$ substrate and on compressively strained Ag layers grown on $\operatorname{Pt}(111)$. The authors established that surface diffusion 


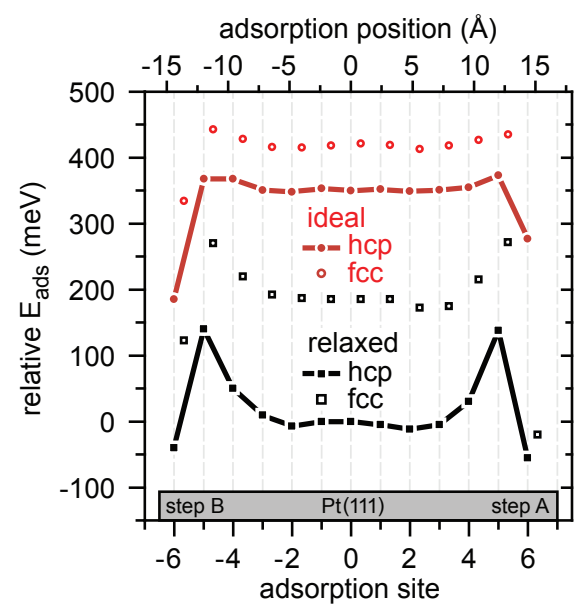

Figure 10: Adsorption energies of a Pt adatom diffusing in a straight line across a $\mathrm{Pt}(111)$ island for a fully relaxed (black squares) and ideal (red circles) geometries. Atom position is given in numbered adsorption sites and Angstrom with respect to the center of the stripe. Integer numbers (filled squares and circles) denote $f c c$ and non-integer numbers (empty squares and circles) correspond to hcp sites. Figure taken with permission from Ref. 91

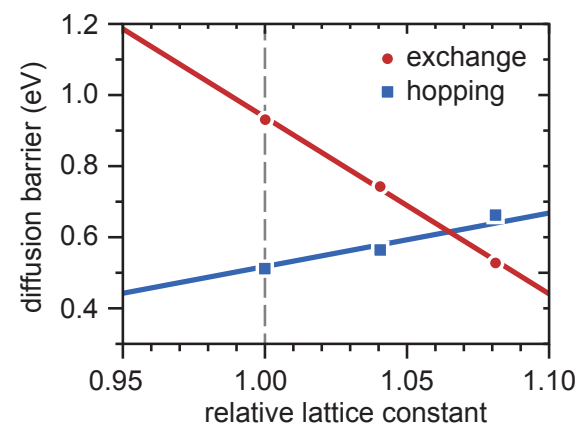

Figure 11: Energy barriers for self-diffusion at a strained $\operatorname{Ag}(100)$ film as a function of the relative surface lattice constant $a_{s}\left(\sqrt{2} / a_{0}\right)$, with $a_{0}=4.14 \AA$ the theoretical lattice constant of silver. Adapted with permission from Ref. 92

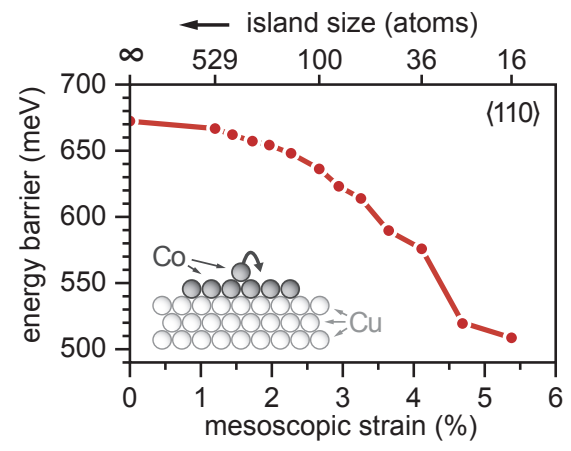

Figure 12: Strain dependence of energy barrier for hopping diffusion on top of Co square islands. Since the strain depends on island size, the activation barrier for diffusion depends also on island size (see upper horizontal scale).

and nucleation are very sensitive to the variation in the substrate lattice constant. Experimentally, the diffusion barrier was observed to be substantially lower on a pseudomorphic strained $\mathrm{Ag}$ monolayer on $\mathrm{Pt}(111)(\sim 60 \mathrm{meV})$, compared to that on unstrained $\operatorname{Ag}(111)(\sim 97 \mathrm{meV})$. The calculations showed that this strong effect is due to the $4.2 \%$ compressive strain of the Ag monolayer on Pt. Further extended theoretical studies [92, 94, 96] showed that atomic motion on strained and unstrained metal surfaces can be characterized by different migration barriers. A general trend found for transition metal surfaces was that compressive (tensile) strains will decrease (respectively increase) diffusion barriers (see Fig. 11) This notion seems to be valid and constitutes an essential factor for kinetic modeling of film growth morphology.

The impact of strain relief on adatom mobility on top of strained Co islands on $\mathrm{Cu}(001)$ and around them was investigated by Stepanyuk et al.. [49] The authors performed atomic scale calculations with ab initio based many-body potentials and showed that atomic motion on strained Co islands is greatly affected by mesoscopic strain relief and depends on the size of the islands. As can be surmised from Fig. 12, the barriers for the hopping diffusion of Co on top of small Co islands (16-50 atoms) are $\sim 20 \%$ lower than those on large islands (100-500 atoms). It was also shown in Ref. 49 that substrate corrugation and ad-structure interaction cause the barrier for edge diffusion of Co atoms along Co islands of any size to be much lower (approximately by $0.1 \mathrm{eV}$ ) than the barrier for Co diffusion on an unstrained terrace. This finding is in agreement with the conclusions attained in kinetic Monte Carlo studies, 97. where it was suggested that the activation energy for stepedge diffusion on an $f c c(001)$ surface is lower than that for isolated-adatom diffusion by hopping.

These findings constitute the first general notion about the interplay of mesoscopic strain and island growth. Preferential diffusion of Co adatoms along the step-edges shall generally promote the formation of compact islands. It also constitutes a possible mechanism of island formation in the absence of thermal mobility. 97 
(a) $\bigcirc \mathrm{Co} \bigcirc \mathrm{Cu}(111) \quad[211]$
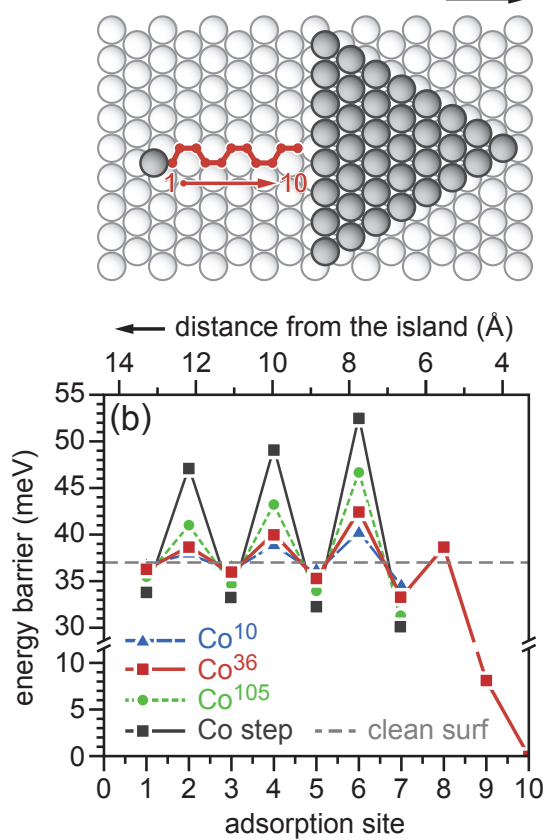

Figure 13: (a) Schematic view of $\mathrm{Co}^{36}$ island (gray circles) and the diffusion path of a Co adatom along which the hopping barriers are plotted in (b). Numbers denote the hops of the adatom. (b) Plot of Co migration barrier near $\mathrm{Co}^{36}$ island. (c) Comparison of migration barriers for Co adatom near triangular Co islands of different sizes and near Co step. Adapted with permission from Ref. 44

The physical mechanism responsible for such pronounced interrelation of strain and diffusion barrier is the variation in the chemical bonding between surface- and adatoms. Tighter packing of the latter reduces the corrugation of the energy landscape which facilitates atomic motion. Taking into account the findings shortly described in Sec. 3.3 we can conclude that island-size-dependent mesoscopic bond contraction is the main reason for the variation of diffusion barriers on top the nano-islands. As already stated, this may and indeed does have important implications for epitaxial growth. For example, since reducing the compressive strain also reduces the diffusivity on top of large islands, a higher nucleation probability is to be expected, which would promote $3 \mathrm{D}$ growth in the early stages of hetero-epitaxy.

Before we move on in our discussion we want to stress again, that while we speak about the impact of strain on diffusion on the meso-scale, the interplay of the two is rather happening on the nano-scale, causing diffusion barriers to vary strongly within a single nano-island, changing the character of diffusion from one adsorption site to the other.

Another flavor of surface diffusion that is important for nanostructure growth and has to be addressed is the diffusion of single adatoms across the surface in presence of ad-structures nearby. We have already seen that diffusion along the edge of a nanostructure is very different from the diffusion on a clean surface, but there is also the scenario of adatom approaching an ad-structure from afar and its incorporation therein. We know that the substrate can be strongly corrugated by the mesoscopic strain relief evoked by the presence of an ad-structure. Wang and Ehrlich have reported finding in their experiments on $\operatorname{Ir} / \operatorname{Ir}(111)$ a "denuded zone", an area around nano-islands devoid of adsorbates, 90, 98] an observation later made for a number of different systems by other groups (see, e.g. Refs. 99, 100). Tsivlin and coworkers 44] have demonstrated theoretically that even a weak corrugation of the substrate can strongly influence diffusion of single adatoms. Atomic scale calculations for $\mathrm{Co}$ islands and steps on $\mathrm{Cu}(111)$ have shown that as the adatom approaches an island or a step on a surface, the migration barrier in the direction perpendicular to the step exhibits strong oscillations (see Fig. 13). The barrier grows as the the adatom approaches the step, forming a repulsive zone which we have briefly mentioned in the introduction of this chapter. 44] Remembering that substrate bond length and stress have strong influence on adatom diffusion, it is not hard to draw parallels between the growing diffusion barrier and the strain relief patterns around nano-islands discussed in Secs. 3.1 and 3.2 .

Since up to now we have only presented examples of monolayer high ad-structures, it is only reasonable to ask oneself whether the observed trends shall hold or change if the nanostructures start to grow in a 3D fashion. To obviate that particular question we turn now to the process of interlayer mass transport and the effect of mesoscopic strain relaxations thereon.

As has been stated above, the two main pathways of interlayer mass transport are over-the-edge jump (inhibited by the Ehrlich Schwoebel (ES) barrier) and the substitutional exchange with the atoms of the ad-structure (see Fig. 15 for a sketch). From a naive point of view, since the ES barrier is determined by the reduced coordination of the adatom jumping over the edge, all possible influences from the strain relaxations should be expected to be higher order corrections. Thus for a long time the ES barrier used to be considered mesoscopic-relaxationindependent. 101 Besides, for the majority of systems the ES barrier is higher than the barrier for exchange interlayer diffusion. The energy of the latter, on the contrary, is directly dependent on the local environment at the step edge and thus highly susceptible to the bond contraction induced by mesoscopic strain relaxations. Studies by Li et al. 101 have established that the ES barrier is indeed almost constant for islands exceeding some tens of atoms in size. However, later studies have shown, that if the islands have a multistoried structure, even the ES barrier becomes susceptible to mesoscopic effects. [102] Yet even more intricate has proven to be the process of exchange transport. The value of the barrier in this case is strongly linked to the static values of adsorption (and desorption) energies on top and beneath the edge of an island or a step.

To attain a better understanding of the process of exchange interlayer mass transport several studies were un- 


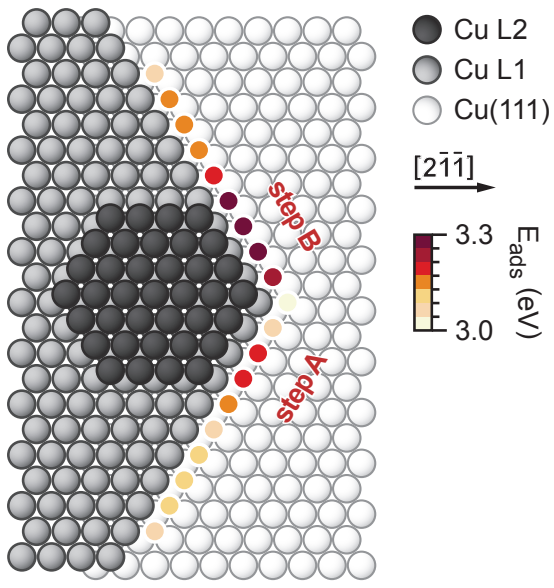

Figure 14: Adsorption energies for a $\mathrm{Cu}$ adatom at the boundary of a double-layer $\mathrm{Cu}$ island for $\mathrm{A}$ and $\mathrm{B}$ step types. Figure adapted with permission from Ref. 37

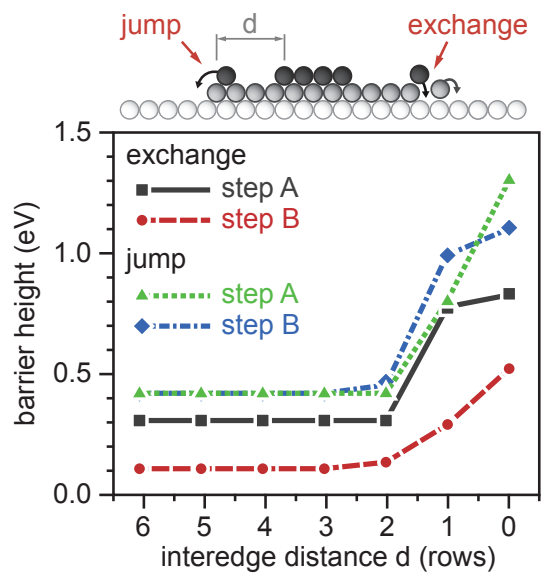

Figure 15: Dependence of energy barriers for hopping and exchange diffusion at $\mathrm{A}$ and $\mathrm{B}$ steps on the distance between edges of the upper and the lower $\mathrm{Cu}$ islands. Figure adapted with permission from Ref. 102

dertaken. The experimental basis for testing the viability of those theoretical predictions were laid by Bromann et al. who devised an STM-based technique for measuring interlayer mass transport characteristics of homo- and heteroepitaxial systems. [103] For the theoretical side, e.g., Lysenko and coworkers 37, 102 have modeled the adsorption energetics in the vicinity of multilayer islands. For doublelayer hexagonal $\mathrm{Cu}$ islands on $\mathrm{Cu}(111)$ it was found that not only does the adsorption energy vary between different step geometries [(100) microfaceted step A and (111) microfaceted step B], but it is also dependent on the presence of the second-layer island (Fig. 14) and its distance from the first-layer step edge (see Fig. 15).

Both the exchange and the ES barriers were shown (Fig. 15) to be distinctly different at $\mathrm{A}$ and $\mathrm{B}$ steps on a (111) surface. This finding was explained by the fact that the average bond length in the vicinity of a B-step is increased compared to an A-step, since the stress relief due to strain relaxations is larger for the latter. Thus, contrary to the picture drawn by Li et al., 101, in the study of Lysenko et al. 37 both exchange and ES barriers were shown to depend on the strain relaxations at the step-edge, especially in the case of $3 \mathrm{D}$ growth. To find a quantitative link between strain and energetics in the 3D growth case Lysenko et al. 37. performed atomic scale calculations for the double layer $\mathrm{Cu}$ island on $\mathrm{Cu}(111)$ when a close contact between the edges occurs. [37, 102] Obtained results demonstrated that mesoscopic strain relief at the edges of islands and in the substrate can lead to a shape transition in the islands as their edges approach each other. Both the upper and the lower islands exhibit strong inhomogeneous strain relaxations: the atoms at the edge of the lower island and the substrate underneath are pushed up, while atoms of the upper island and the substrate under the large island are pushed down. Mesoscopic misfit between the upper island and the lower island were found to depend on the distance between the edges and lead to a different local distortion of the adsorption potential around the cluster edge steps of A and B types. As a consequence, adsorption energies for $\mathrm{Cu}$ adatoms near the edge of a double layer islands, as shown in Fig. 14 , exhibit large variation of more than $0.3 \mathrm{eV}$ (about 10\%) for both steps A and B. The physics behind is straightforward: the shorter bond lengths at A-steps lead to a reduced corrugation of the potential acting on adatoms as compared to B-steps, which, in turn, as already remarked earlier and pointed out by $\mathrm{Yu}$ and Scheffler, 92 changes the corrugation of the binding potential and consequently the adsorption energies along with the barrier for interlayer mass-transport. The corrugation changes as the secondlayer island approaches the step edge and the interlayer transport barriers change accordingly.

The effect is, of course, not limited to $\mathrm{Cu}(111)$. Similar differences were found in calculations for $\mathrm{Ag}(111)$ and Pt(111), where the barrier for exchange turned out to be considerably lower at a B-step than at an A-step. 101, 104

Thus the scenario of the interlayer mass transport is dramatically changed by the presence of a second-layer cluster close to the border of the first-layer island. The downward movement of atoms at the edge is strongly suppressed in both ES and exchange channels (see Fig. 15). The barrier heights involved in the mass transport at both A- and B- type steps are strongly increased by the presence of the second-layer ad-structure, which is, of course, due not only to the mesoscopic relaxations, but also to the direct interaction of the adatom with the ad-structure itself.

It must be noted, however, that for a process of such complexity it is hard to deduce a truly universal rule which would be devoid of exemptions. A number of early STM studies by Giesen and coworkers [105 107] dealing with multilayer $\mathrm{Cu}$ islands on $\mathrm{Cu}(111)$ have shown that when the distance between the edges of the upper and the lower islands is small, the decay rate of small islands increases by two orders of magnitude. Originally, Giesen and coworkers have tried to explain their observations by the disappear- 


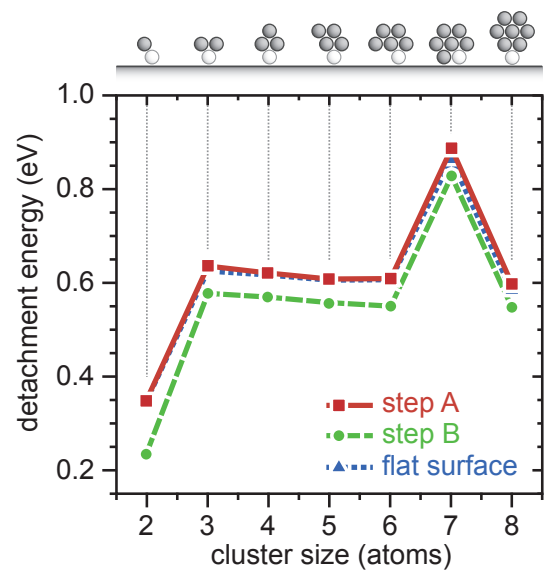

Figure 16: Detachment energies for atoms of small stable $\mathrm{Cu}$ clusters at the step edges of $\mathrm{Cu}(111)$ and on the flat substrate; the step position is shown for a close contact between clusters and the step. Not the that the "flat surface" curve is almost completely obscured by "step A" data. Figure adapted with permission from Ref. 102

ance of the ES barrier, which would contradict the conclusions of the studies by Lysenko et al. Yet it was suggested later, after similar results have been obtained for small and large $\mathrm{Ag}$ islands on $\mathrm{Ag}(111)$ by other groups, [108, 109. that the apparent discrepancy can be explained by the existence of a different atomistic process, namely a collective exchange at the step edge, promoting interlayer mass transport and the resulting second-layer cluster decay. [108] A more thorough atomistic description of the phenomenon was given by Lysenko et al., who's atomicscale simulations have shown that the reduced detachment energies at the step edges are a result of the joint influence of reduced coordination and mesoscopic strain relaxations. In Fig. 16 the detachment energies of $\mathrm{Cu}$ atoms from small $\mathrm{Cu}$ clusters at A- and B- type steps are compared with those for the flat surface. One can see, that the influence of an A step on the detachment energies is very small, while at a B step the energies of all clusters are reduced compared to the flat surface by $60-70 \mathrm{meV}$.

Having established that both intra-layer and inter-layer diffusion are affected by the mesoscopic strain relaxations in adsorbate islands, we want to spend a few lines on a similar class of ad-structures - embedded clusters. It turns out that in this case the mesoscopic effects are equally important. For instance, performing atomic scale calculations with ab-initio-based many-body potentials, Longo et al. 110 have shown that sub-monolayer inclusions of Fe atoms in the topmost layer of $\mathrm{Cu}(001)$ observed in experiments 111, 112 are caused by a collective process of atomic exchanges near small embedded Fe islands. They demonstrated that mesoscopic relaxations in the substrate depend on the size of embedded islands and strongly affect site exchanges. Fig. 17(b) shows how the exchange barrier for the $\mathrm{Fe}$ adatom depends on the distance to Fe islands embedded in a $\mathrm{Cu}$ substrate. Performed calculations revealed a strong reduction of the exchange barrier near the (a)
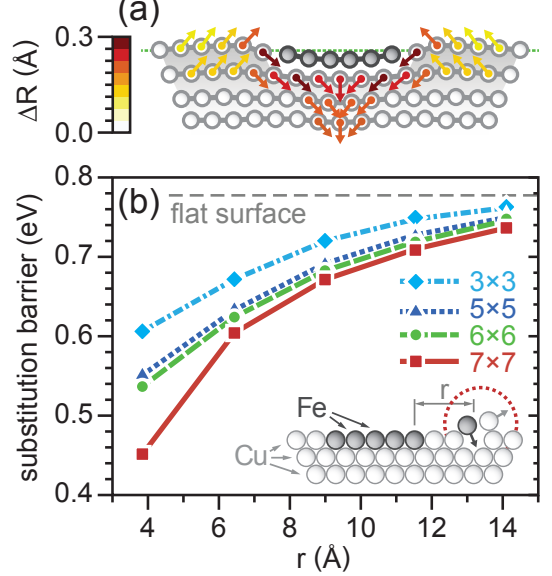

Figure 17: (a) The shape of an embedded $\mathrm{Fe}^{36}$ and the $\mathrm{Cu}$ substrate (the dotted line represents the surface level). Arrows show the direction of the displacement of the atoms in the system and their colour encodes the displacement magnitude. (b) Substitutional exchange barrier for a $\mathrm{Fe}$ adatom on a $\mathrm{Cu}(001)$ surface near an embedded square Fe island. The horizontal line represents the exchange barrier for a flat surface. Figure adapted with permission from Ref. 110

island. This effect is caused by the increased interaction between the Fe adatom and the island at short distances (the main point is the number of atoms of the island with which the adatom interacts) and by the local strain relaxations. In the present case, a strong inhomogeneous strain distribution in a $\mathrm{Cu}$ substrate around embedded Fe islands was found (see Fig. 17(a)). The surface layer and the embedded $\mathrm{Fe}^{36}$ island are strongly corrugated due to atomic relaxations. The substrate atoms, which are the nearest neighbors of the island, are strongly pushed down, while more distant atoms exhibit upward relaxations. Substrate atoms are also strongly shifted in-plane of the surface following the tensile stress acting on the substrate they relax towards the island. Of course, due to atomic relaxations the bond lengths in the substrate in the vicinity of the Fe island are increased compared to the flat surface. According to the results of $\mathrm{Yu}$ et al. 92 those longer bond lengths lead to an enhanced corrugation of the potential acting on the adatom and reduce the exchange barrier (see Fig. 11. As a direct consequence of the decrease of the exchange barrier, the probability of a collective intermixing mechanism compared to another intermixing processes is enhanced. To give a particular example, at room temperature the probability for the intermixing directly near an embedded $\mathrm{Fe} / \mathrm{Cu}(001)$ island of 36 atoms was found to be more than ten times larger than the probability of this process 6-7 $\AA$ away from the island. [110] This difference is even bigger if one compares it with the edge diffusion along the embedded island or with exchange on a flat surface. Therefore, one can conclude that the exchange process near embedded islands is the mechanism of the formation of $\mathrm{Fe}$ inclusions in $\mathrm{Cu}(001)$ found in experiments. Naturally, also the different dynamics of step atoms (also due to different holding potentials) makes penetration and 
incorporation of foreign/impurity atoms particularly easy at step edges (2D alloying into upper terraces).

\subsection{Mesoscopic mismatch as a driving force for morphol- ogy modification at the atomic scale}

Having established beyond doubt the importance of mesoscopic misfit for nanostructure growth (adatom mobility and nucleation kinetics) we turn back to a topic superficially scratched in Sec. 3.4 - the interplay of mesoscopic mismatch and the morphology of metallic heteroand homo-epitaxial systems.

Of course, a topic of particular interest in this case is the growth of strained islands on metal surfaces due to its fundamental and practical interests. It has been shown in the framework of classical theory of elasticity, [28, 86, that in equilibrium the competition between the line tension (which favors a compact shape) and strain energy may lead to a spontaneous shape transition from compact to elongated islands above a critical island size. [35, 113116. STM measurements of Müller et al. 35, [115, 116. demonstrated that strain relief may lead to ramified island growth in sub-monolayer hetero-epitaxy of $\mathrm{Cu}$ on $\mathrm{Ni}(001)$ (see Fig. 8). The shape transition from compact to ramified islands above a critical size, which was predicted theoretically by Tersoff and Tromp, 28, has been observed over a wide range of temperatures $(\mathrm{T}=250-370 \mathrm{~K})$ and deposition fluxes $\left(10^{-5}-10^{-2}\right.$ monolayer/s). It was suggested that this shape transition does not have a kinetic origin and is determined by the island-size-dependent strain relaxation of step-edge atoms due to the positive lattice mismatch. Recently Shim et al. [117] presented results of kinetic Monte Carlo (kMC) simulations which indicate that the shape transition is not primarily determined by energetic effects, but instead is due to kinetics being strongly affected by strain. Tersoff predicted that, in general, the interface intermixing can be expected in systems dominated by atomic size mismatch. [118] This straindominated scenario is consistent with a variety of experimental findings. Ex situ atomic force microscopy (AFM) observations of Zimmermen et al. 119] have shown that Co particles "burrow" into clean $\mathrm{Cu}(001)$ and $\mathrm{Ag}(001)$ substrates at $600 \mathrm{~K}$, while no burrowing was observed at room temperature. Padovani et al. [120] have reported that cobalt clusters "submerge" into the $\mathrm{Au}(111)$ surface at a temperature about $450 \mathrm{~K}$. These experiments revealed a novel mechanism of mass transport in transition metal hetero-epitaxy, which changes the surface morphology of the growing films. To gain insight into the origins of burrowing Stepanyuk et al. [121] performed atomistic simulations. Based on the concept of mesoscopic misfit he proposed that the coating of Co islands by highly strained substrate material involves vacancy formation around the structure and leads to a large pressure at the $\mathrm{Co} / \mathrm{Cu}$ interface which can promote burrowing. [121]

Another example of mesoscopic misfit affecting the morphology and dynamics of surface structures was given by Miron and coworkers [51, who employed an accelerated
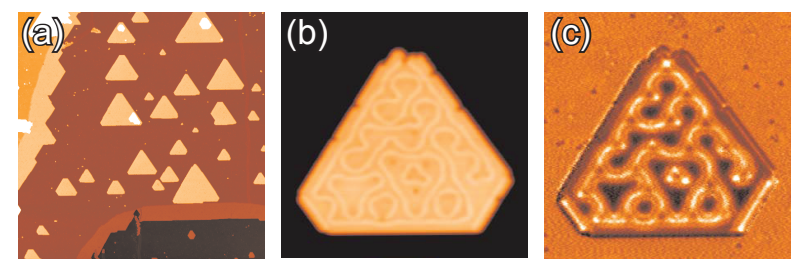

Figure 18: Co monolayer islands and wires prepared by Co deposition on the $\mathrm{Pt}(111)$ surface at room temperature. Overview STM map (a) and a more detailed topography of a single island (b). dI/dV map (c) measured simultaneously to the topographic image in (b) with an out-of-plane sensitive magnetic tip. Figure adapted with permission from Ref. 122

molecular dynamics simulations to study atomistic processes involved in the growth of $\mathrm{Co}$ on $\mathrm{Cu}(001)$. They observed an upward interlayer transport at island edges that was responsible for the bilayer Co island formation at low temperatures. At higher temperatures the $\mathrm{Co}-\mathrm{Cu}$ intermixing on the terrace produced qualitatively different island morphologies, leading to downward interlayer transport and thereby promoting layer-by-layer growth. Furthermore, Miron et al. have shown that strong strain relief in Co islands on a $\mathrm{Cu}$ surface leads to a high mobility of small Co clusters through concerted atomic mechanism that significantly lowers the saturation island density and thus affects the low coverage morphology in agreement with experimental observations. 51.

A detailed experimental insight into the growth, structure and morphology of thin Co layers deposited at room temperature on $\mathrm{Pt}(111)$ has been achieved through the use of scanning tunneling microscopy. [100, 122,125] It was found that the strain relief within the first and second Co layers changes the energy balance between hollow sites $(f c c$ and $h c p$ ) and the bridge sites in the system due to the large lattice mismatch of $9.4 \%$ between Co and Pt. It thereby promotes the formation of a dislocation network. 123, 125] Later these dislocations relieve the strain through a formation in the second Co layer of a hexagonal "moiré" structure with the Co in-plane lattice distance being close to that of bulk Co (see Fig. 18). STM measurements [122, 124] demonstrated, that the growth of thin Co film is followed by flat layers (2D) up to a Co coverage of about 3.5 ML. Above this coverage 3D Co islands are observed. It was argued, that the driving force of $2 \mathrm{D}-3 \mathrm{D}$ growth behavior is a balance between strain energy at the interface between Co layers and Pt surface and the bond energy of the islands' Co atoms which determines the surface morphology of a growing structure. This study is also a vibrant example of how mesoscopic strain relaxation can affect not only the morphology (Fig. 18(b)) but also the magnetic properties (Fig. 18(c)) of a growing nanostructure layer.

Another STM investigation worth mentioning is a study by Donati and colleagues of the strain mediated nucleation and growth morphology of $\mathrm{Fe}$ on $\mathrm{Au}(111)$ occurring in the sub-monolayer regime at room temperature. 67, 126 It is 


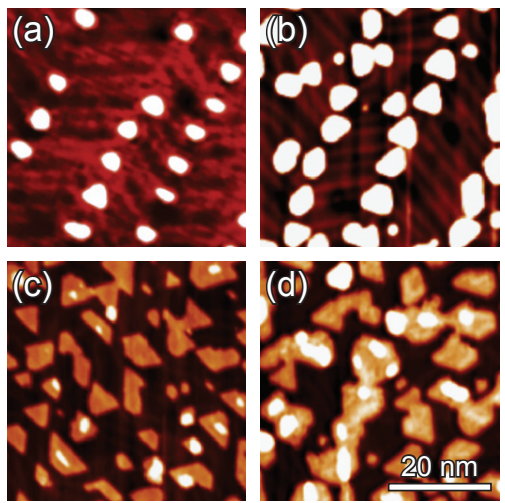

Figure 19: STM images of $0.1,0.2,0.4$ and $0.6 \mathrm{ML}(\mathrm{a}-\mathrm{d})$ of $\mathrm{Fe}$ deposited on $\mathrm{Au}(111)$ surface. Figure adapted with permission from Ref. 126

well known that the $\mathrm{Au}(111)$ surface exhibits a uniaxial reconstruction which shows $f c c$ and $h c p$ regions separated by discommensuration lines (ridges), while rotational domains of $\pm 120^{\circ}$ generate peculiar sites (so-called bulged and pinched elbows) of the reconstruction. [127 The interatomic distances are different in these regions and induce the non-uniform surface strain that makes $\mathrm{Au}(111)$ surface an ideal template for nucleating metal (Ni, Co, Fe) clusters in well ordered arrays. 67, 126] As an example of how this strain can be used to tune the morphology of the growing film in Fig. 19 the growth scenario for $\mathrm{Fe}$ on $\mathrm{Au}(111)$ is presented as a sequence of STM images taken by Donati et al. [67, 126] As can be seen in Fig. 19(a), below a coverage of $0.1 \mathrm{ML}$, Fe growth proceeds in well ordered regular arrays of monolayer-thick islands nucleating preferentially at elbows sites. It is shown, that the bulged elbows are covered by triangular shaped islands, while pinched elbows favor the diamond shaped ones. Such peculiar growth was explained by the presence of non-uniform reconstruction-induced surface strain. However, the situation is rather different for coverages of 0.2-0.4 ML (Figs. 19(b) and 19(c)). A few Fe islands grow out of the elbows sites, while a fair number of Fe islands have coalesced forming larger islands with elongated shape (in a quasi 1D fashion). Most of the islands remain well separated, showing a tendency to grow perpendicularly to the elbows line. A small but non-negligible fraction of $\mathrm{Fe}$ begun to grow also in the second layer of the islands. Coalescence and second layer growth are instead more evident for a coverage of 0.6 ML (see Fig. 19.(d and e)). These STM observations of morphology evolution in $\mathrm{Fe} / \mathrm{Au}(111)$ were interpreted successfully through rate equation models for the island densities including the explicit assumption of Fe-Fe bond length (energy) dependence on the island size.

At the end of this section, we shall give yet another theory-based example of how strain relief affects the growth morphology of deposits on substrate. Combining kinetic Monte Carlo (kMC) and molecular static simulations with ab initio based interatomic potentials, Negulyaev et al. 62.

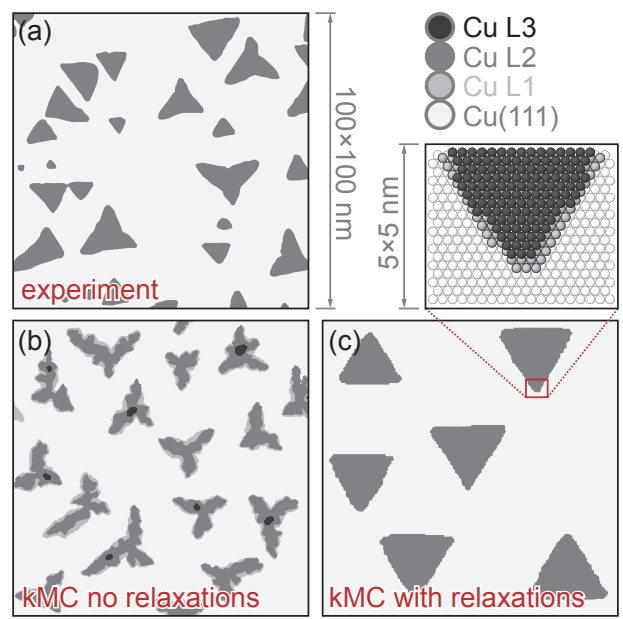

Figure 20: The morphology of $\mathrm{Cu}(111)$ surface exposed by $0.4 \mathrm{ML}$ of Co at $290 \mathrm{~K}$ : (a) experimental STM map (darker areas have larger apparent height) and the kMC simulations neglecting the strain relaxations (b) and properly taking them into account (c). The inset in the upper right panel shows the atomic scale structure of a section of a double-layer island as obtained in kMC simulations. Figure adapted with permission from Ref. 62

explored, on the atomic scale, the formation of triangular bilayer Co islands on $\mathrm{Cu}(111)$ at room temperature (RT) benchmarking the results against experimental observations. According to the latter, at $290 \mathrm{~K}, 0.4 \mathrm{ML}$ Co deposited on $\mathrm{Cu}(111)$ coalesce into triangular islands of regular shape, well separated from one another (Fig. 20(a)). Taking all relevant atomistic processes into account, yet neglecting the mesoscopic relaxations in the system, detailed kMC simulations predict a totally different, dendrite structure of the island $(20(\mathrm{~b}))$, characteristic for much lower experimental growth temperatures. Yet once the mesoscopic effects are introduced into the model (Fig. 20(c)) the resulting island shapes become almost a perfect match to the experimental ones. These results explicitly and unambiguously demonstrate that the strain relaxations have a role of paramount importance in the determination of epitaxial morphology at the atomic scale.

The last example that we shall bring of the importance of mesoscopic strain relaxations in early stages of epitaxy is a study of low-temperature self-ordering of $\mathrm{Fe}$ adatoms on $\mathrm{Cu}(111)$ carried out by Negulyaev et al. 65. In it the authors have shown, in a joint experimental (STMbased) and theoretical (kMC) investigation, that strain relaxations induced in the substrate have a pronounced effect on the energy landscape of Fe adsorbate diffusion. Measurements for different temperatures and coverages have shown that strain relaxations substantially increase the probability of close-packed cluster formation during the early stages of metal-on-metal epitaxial growth. As a signature of the importance of mesoscopic misfit the authors present the density of immobile clusters $(\geqslant 3$ atoms) created at annealing temperature of $12 \mathrm{~K}$ as a function of the coverage (Fig. 21). Four different coverages were considered: $0.011,0.021,0.031$, and $0.048 \mathrm{ML}$. One can see that 


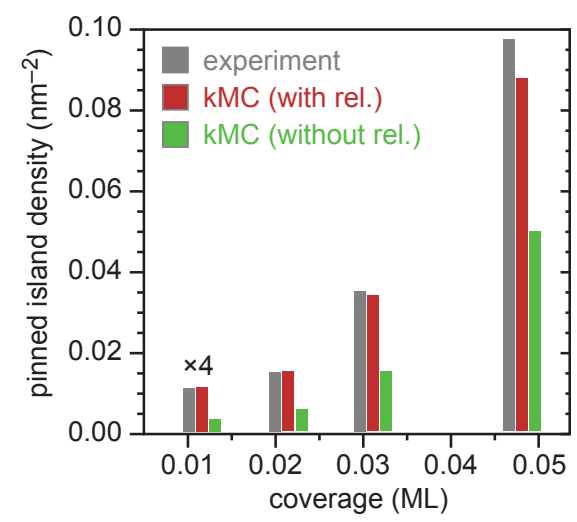

Figure 21: The density of immobile Fe islands on $\mathrm{Cu}(111)$ surface as a function of coverage at annealing temperatures of $12 \mathrm{~K}$ as measured experimentally (dark gray) and calculated with (red) and without (green) taking into account strain relaxations. Figure adapted with permission from Ref. 65

the concentration of immobile clusters measured in the experiment and that obtained with the kMC simulations on a fully relaxed substrate are close together. The nucleation density of clusters found in the kMC simulations while neglecting strain relaxations is significantly lower, differing often by a factor of more than two from the experimental values.

\subsection{Local variation of electronic properties in strained nano- islands}

While the foregoing section was almost exclusively devoted to the issues of morphology and the effect of mesoscopic misfit thereon, in it we have already caught a glimpse of the effect that misfit-induced reconstruction can have on the electronic and magnetic properties of the system (see Fig. 18 and Ref. 122). In the coming two sections we shall present further evidence that such interplay is not a pure coincidence, but is rather ubiquitous.

In contrast to epitaxial atomic layers, where lattice misfit, epitaxial strain, vertical layer relaxation are well defined properties [10, 87, 128, for individual nano structures spatial variations of the atomic structures are expected [41, 43, and experimentally observed [31, 61, 129]. Here, structural and electronic relaxations on the nanoscale are driven by uncompensated forces and reduced coordination at the boundary of a nano structure. As a result, both atomic positions and electronic structure show a pronounced spatial dependence [58, 67, 130. Thus, any appropriate description of spin-dependent properties stringently requires the consideration of these effects. Note, that concepts such as strain and layer relaxation need to be applied with considerable care, as the atomic distances vary throughout a single nano structure, and the description by a oversimplified strain concept appears questionable. Rather, the description in view of mesoscopic misfit [37, 43] appears to be better suited to address the peculiar bonding situation in nanostructures.
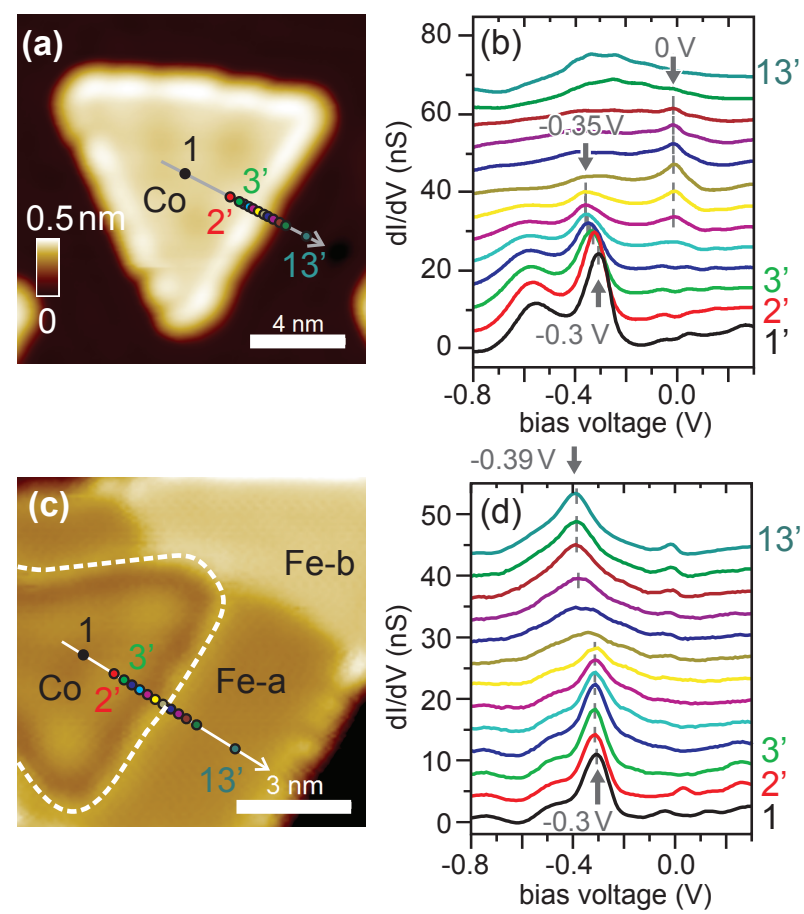

Figure 22: Topography and spatially resolved spectroscopy of pure Co (top row) and Fe-decorated bilayer Co islands (bottom row). Constant current STM images of a pure Co bilayer island in (a) and of a Fe-decorated Co island in (c) $\left(U_{\text {gap }}=+0.1 \mathrm{~V}, I_{\mathrm{t}}=1 \mathrm{nA}\right)$. The arrow in (a) and (c) shows the line along which position dependent spectroscopy data were taken in (b) and (d), respectively. The spectra are shifted vertically for clarity. Whereas the data for pure Co show a sizable shift of the peak at $-0.3 \mathrm{eV}$ to more negative values to $-0.35 \mathrm{eV}$ upon transition towards the edge of the island. The peak position measured on the Fe-decorated Co island does not show any appreciable shift and remains at $-0.3 \mathrm{eV}$. See text for the discussion. 

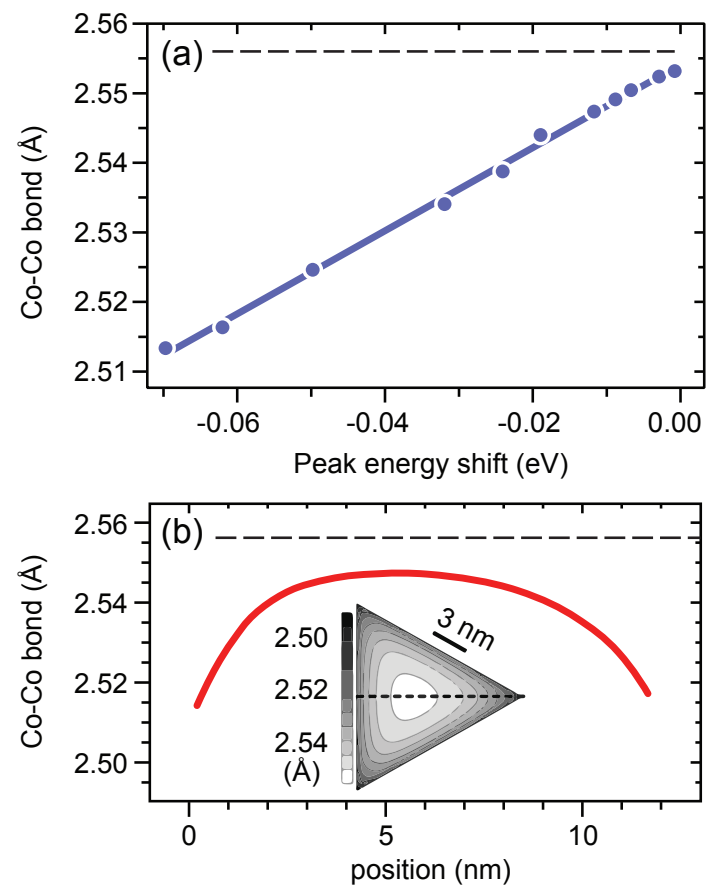

Figure 23: (a) Relation between the average Co-Co bond length of a bilayer Co structure on $\mathrm{Cu}(111)$ and the shift of peak energy of the $3 d$ minority state of Co. The data are extracted from a previous study. [130] The dashed line indicates the Co bond length of $2.556 \AA$, representing pseudomorphic growth. The blue line is a guide for the eye. The peak energy shifts to more negative values with decreasing bond length. (b) Plot of the calculated average Co-Co bond length in a single bilayer $\mathrm{Co}$ island on $\mathrm{Cu}(111) \quad 130$ along the dashed center line. The bond length varies by $0.003 \mathrm{~nm}$, and it is the shortest at the island edge. It remains below the value indicative of pseudomorphic growth (dot-dashed line) even at the island center. Figure adapted with permission from Ref. 130

Here we exploit the spatial resolution of the STM to investigate the position dependence of the differential conductance signal [130, 131] within pure and Fe-decorated Co islands. These data provide compelling evidence that structural relaxations in the Co core are lifted upon decoration.

The following Section provides experimental evidence that spin-polarization and magnetic anisotropy in the rim region of individual Co bilayer islands are drastically changed upon perimetric decoration. The spin-polarization becomes spatially more homogeneous, and the magnetic anisotropy of the Co core is reduced upon Fe decoration.

The data of Fig. 22(b) reveal that the energy position of the peak of the differential conductance, which is related to the Co minority states, located near $-0.3 \mathrm{eV}$ at the island center, first reported and identified by Diekhöner and coworkers, 132 shifts to more negative values towards $-0.4 \mathrm{eV}$ close to the island edge. 130. Near the island edge, a novel electronic feature appears in spectroscopy as a peak at the Fermi energy, which has been coined rim state. 133. Where it is observed, the intensity of the peak near $-0.4 \mathrm{eV}$ is strongly suppressed.

We ascribe this spatial dependence of the spin-dependent electronic structure in an individual nano island to the impact of both, lattice relaxation and reduced coordination. Previous combined experimental and theoretical studies have identified the intimate link between structural relaxation and electronic structure for this [130] and a related system [67, and we focus on the structural relaxation first.

The observed shift of the peak energy to more negative values towards the island edge for the island shown in Fig 22(a) with a base length of $12 \mathrm{~nm}$ can be ascribed to a corresponding reduction of the in-plane Co-Co atomic distance. We present the relation between the average Co-Co bond length of Co islands of different size and the peak energy shift in Fig. 23(a), as extracted from the published data. [130] The plot shows as a dashed line the Co-Co bond length of $2.556 \AA$, which serves as a reference indicating the bond length in pseudomorphic positions on $\mathrm{Cu}(111)$. At this bond length a peak energy of $-0.3 \mathrm{eV}$ is observed. The plot reveals that a shift of peak energy to more negative values indicates a shorter Co-Co bond length.

The calculations indicate that the Co-Co bond length at the center of a $15 \mathrm{~nm}$ size island approaches the value indicative of pseudomorphic growth on $\mathrm{Cu}(111)$. This gives a nearest neighbor distance of $\frac{1}{\sqrt{2}} a_{\mathrm{Cu}}=2.556 \AA$, with $a_{\mathrm{Cu}}=3.615 \AA$. However, near the island edge a reduced bond length of $\approx 2.51 \AA$, is found in the calculations. This reduced bond length leads to a peak energy shift by $-0.07 \mathrm{eV}$. The structural relaxation reflects the tendency of epitaxial misfit stress to reduce the Co-Co bond length. This stress is driven by the tensile misfit of $\eta=\left(a_{\mathrm{Cu}}-a_{f c c-\mathrm{Co}}\right) / a_{f c c-\mathrm{Co}}=+1.89 \%$, with $a_{f c c-\mathrm{Co}}=3.548 \AA$. [134] As a result, atomic positions change throughout the nano structure, as stresses acting at the boundary of the structure differ from those acting at the island center, and this drives a spatially varying structural relaxation, which affects both topmost and the interface layer in contact with $\mathrm{Cu}$. Note that topmost and interface Co atoms may respond differently to the stress, and the notion of a strain to characterize the bond length on individual $\mathrm{nm}$ small islands may be too simplistic. Thus, a complex atomic relaxation is expected, and it is linked to the spatial dependence of the electronic structure, as observed here. The variation of the Co-Co bond length within a single Co bilayer island is shown in Fig. 23(b). Note that in this small Co island, all Co-Co bond lengths remain below the value expected for pseudomorphic growth.

A further contribution which modifies structural and electronic properties is the reduced coordination. The lack of bonding partners inevitably affects all atoms at the boundary of any structure. Whereas an atom within a fcc material is surrounded by twelve nearest neighbors, this coordination shrinks to nine for atoms at the (111) surface, and to seven for an atom at the ridge of an (111) island. This reduced coordination leads to an electron redistribution. This influences the atomic spacings, which often get shorter, and it gives rise to surface stress, which is gener- 
ally tensile 135, 136. Thus, the position dependence of the electronic properties, and consequently of the atomic structure, is a general phenomenon for nano structures, and it is not limited to epitaxially strained systems. 37.

The decisive difference of the position dependent spectroscopy data in Fig. 22 (d) as compared to the data shown in (b) is an almost constant energy position of the spectroscopy signal from the Co core towards the border upon Fe decoration. This contrasts with the substantial shift in peak energy for the corresponding transition from the center towards the edge of the pure Co island. Also, the electronic signature of the rim state as a peak at the Fermi energy is only observed for the pure Co island, but not for the Fe decorated island.

These observations support the conclusion that structural and electronic relaxation of a pure Co islands are substantially suppressed in the Co core of the Fe-decorated island. We discuss in the following section, that also the magnetization reversal and magnetic anisotropy of the Co islands are heavily affected by the decoration, leading to a reduced magnetic anisotropy of the Co core upon decoration. We ascribe the underlying physics to the effect of suppressed structural and electronic relaxation upon contact of the Co edge with Fe.

\subsection{Influence of mesoscopic relaxation on the magnetism of nano-islands}

Lattice strain and magnetism are intimately linked by the magneto elastic coupling, which describes the strain dependence of the magnetic anisotropy [10, 68, 137, 138. In short, an anisotropic lattice strain is often the driving force for a change of the easy axis of magnetization in epitaxial layers, away from the respective bulk direction. In epitaxial films, the in-plane and out-of-plane lattice strain differs in general [10, 138, and the magneto elastic coupling between strain and magnetic anisotropy may drive the spin reorientation.

However, although the concepts of lattice strain and magneto elastic coupling are well established for bulk samples and atomic layers, the corresponding description of individual nano structures has not been established yet. It is evident that in view of the spatial variation of bond lengths in a single nanostructure on the nm scale, the concept of lattice strain is questionable. Consequently, the reliable application of magneto elastic concepts to individual nano structures appears debatable. Nevertheless, it is expected that even subtle structural relaxation will have a dramatic impact on the spin-dependent electronic properties. And as a result, also magnetic properties, such as the magnetic anisotropy, will be influenced by structural relaxation on the nm scale.

In this section we present an intriguing example for the suspected role of relaxation in individual Co bilayer nanoislands on $\mathrm{Cu}(111)$. We find that the magnetic anisotropy of the Co island varies within a single island. Our quantitative analysis of magnetization reversal data by spin-STM provide compelling evidence that the atoms near the edge
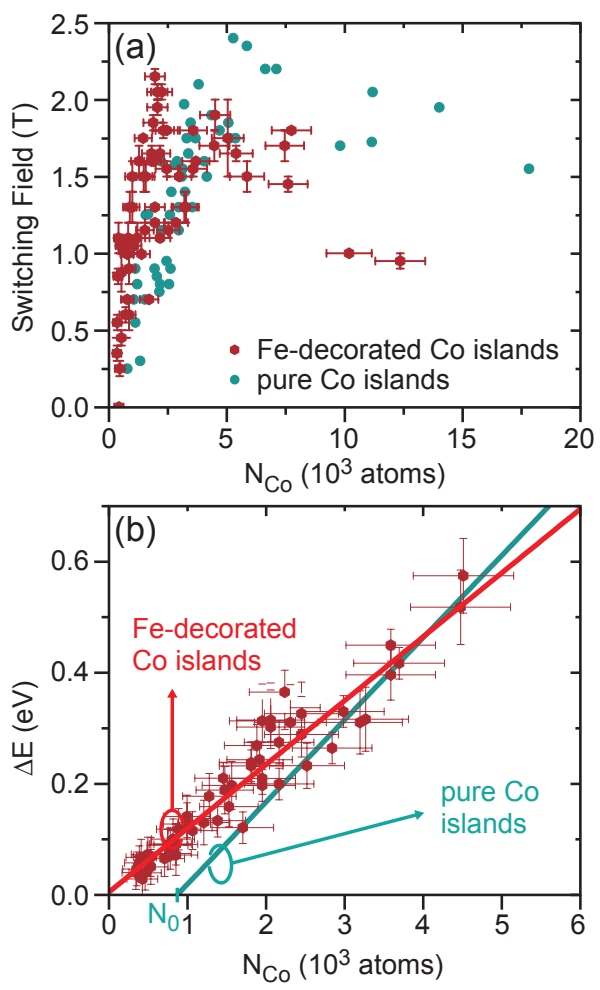

Figure 24: (a) Summary of switching fields of individual bilayer Co islands, as measured by in-field spin-STM at $8 \mathrm{~K}$. The data points of pure Co (cyan) islands show smaller switching fields as compared to Fe-decorated Co (red) islands. The switching field was measured for a variation of the magnetic field along the sample normal. (b) Energy barrier $\Delta E$ of magnetization reversal in dependence of island size given by the total number of Co atoms $N_{\mathrm{Co}}$. The data for pure Co islands are described by a linear fit, and only this line (cyan) is shown for clarity. The complete description is given in. 69 The linear fit shows an offset $N_{0}$ at the intersection with the horizontal axis. The data of Fe-decorated Co islands (red) extrapolate through the origin of the plot, and they are well described by a linear relation. The slopes of the linear fits give the magnetic anisotropy of the systems.

of the Co bilayer island are magnetically soft, i.e. they exhibit a vanishing magnetic anisotropy [69, 71, 131]. We are led to the proposition that structural relaxation at the island edge is a pivotal aspect of this peculiar behavior. This view is corroborated by our recent experiments which indicate a lifting of structural relaxation upon perimetric decoration of the Co islands with a bilayer Fe stripe. 131. As a result, now all atoms of the Co core show a magnetic anisotropy.

We exploit the spatial resolution of spin-polarized STM to investigate the magnetization reversal of individual bilayer $\mathrm{Co}$ islands on $\mathrm{Cu}(111)$. These $\mathrm{Co}$ islands have an easy out-of-plane magnetization direction. [139] We measure the switching field for islands of different size from the sharp signal drop of the differential conductance during a sweep of the magnetic field 69, 71.

Figure 24(a) shows a compilation of the data. Switching field data are plotted for pure (cyan) and Fe-decorated (red) Co islands. The data show an initial sharp increase 
of the switching field with island size, and the switching field decays to smaller values in larger islands. This overall behavior is ascribed to thermally assisted magnetization reversal 69, 140. In smaller islands the total magnetic anisotropy energy of the island is comparable to the thermal energy, leading to thermally assisted magnetization reversal. In larger islands, the total magnetic anisotropy energy is larger, the thermal energy gets comparably less important, and the switching field approaches the StonerWohlfarth value of $2 K / M$, where $K, M$ are the magnetic anisotropy and the magnetization, respectively. [141] In this thickness range magnetization reversal by coherent rotation of a so-called macro-spin - all spins of the nanostructure rotate in unison - is assumed. In even larger islands magnetization reversal by the nucleation of magnetic domains and domain growth is feasible, and smaller switching fields are observed. 69] We note that the data for the Fe-decorated islands show larger switching fields as compared to pure Co islands up to an island size of 2500 atoms.

We apply the analysis of the switching field data in the framework of the Néel-Brown description of thermally assisted reversal to extract the energy barrier $\Delta E$ of reversal. 69 The result is shown in Fig. 24(b). The plot shows the result for the pure Co islands as a line fit (cyan) through the data points, where the individual points are omitted for clarity. The slope of the line gives the magnetic anisotropy of $0.148 \pm 0.005 \mathrm{meV} /$ atom. Surprisingly, this line shows an offset, and it intersects the x-axis near 870 atoms. We ascribe this to the number of atoms, which do not contribute to the magnetic anisotropy. It appears plausible to localize the atoms of vanishing magnetic anisotropy at the rim of the island, within a stripe of width 1-4 atoms. 69.

Previously, it has been proposed that edge atoms exhibit a higher magnetic anisotropy, due to their reduced coordination. 142 Our present work shows that coordination effects are comparably less important. Rather, the magnetic properties of Co islands on $\mathrm{Cu}(111)$ appear to be affected by structural and electronic variations near the island edge.

The data for the Fe-decorated island show a very different behavior. The slope of the curve representing a linear fit through the data points indicate a magnetic anisotropy of $0.115 \pm 0.005 \mathrm{meV} /$ atom, $22 \%$ percent less than for pure Co islands. Remarkably, the fit extrapolates to zero, and there is no indication of a sizable offset. This is in sharp contrast to the result obtained for the pure Co islands.

How can we understand this change of the magnetic anisotropy of the Co island upon decoration? We ascribe this change of the magnetic anisotropy to the decorationinduced lifting of the structural relaxation near the edge of the Co core and to the corresponding change of the electronic structure, as discussed above in Section 3.7 .

Finally, a few words have to be said about the predictive power of theoretical calculation. In fact, performing $a b$ initio and tight-binding calculations Pick and coworkers demonstrated that atomic relaxations can affect magnetic properties of $\mathrm{Co}$ adatoms and $\mathrm{Co}$ clusters on a $\mathrm{Cu}(001)$ surface. 143] Atomic relaxations decrease the spin and orbital magnetic moments and drastically affect the magnetic anisotropy of the Co adatoms. Due to relaxations the in-plane magnetization of the Co adatom is stabilized. 143.

In another study it was proven, that atomic relaxations strongly reduce the magnetic anisotropy energy (MAE) and the orbital magnetic moments of embedded clusters (see Fig. 2(f) for a sketch of predicted relaxation). The largest MAE of about $1.8 \mathrm{meV}$ was found for a single Co atom in the $\mathrm{Cu}(001)$ surface. A strong enhancement of the spin magnetic moments in embedded clusters as compared to a single atom of $\mathrm{Co}$ incorporated in the $\mathrm{Cu}(001)$ surface was found as well. While in supported clusters the MAE is strongly enhanced at the edge atoms, the immersion of the cluster into the surface and atomic relaxations make the distribution of the local MAE contributions and orbitalmoment values almost homogeneous. 144.

\section{Experimental confirmation of mesoscopic mis- match I: Surface x-ray diffraction}

The discussion of the previous sections has elaborated the most important aspects regarding the modification of the structure of ultra thin films, namely the considerable reduction of the interatomic distances within a nanoisland. It should be noted that the phenomenon of a bond distance contraction is quite general and is well known for simple metal surfaces. 145 In simple picture it can be repylated to the Smoluchowski smoothing [146] of the surface charge density contour involving a electrostatically driven inward relaxation. In another model according to Pauling [19] the reduced number of neighbors around a given atom leads to a strengthening of the remaining bonds and as a consequence to the reduction of the bond length. Following this idea any kind of modification of the environment around an atom - especially at the rim and at edged nanoislands or clusters- is expected to lead to substantial modifications of the atomic structure. It was already in 1951 that the first experimental verification of the particle size effect on the lattice parameter was presented by Boswell et al. 20, in an electron diffraction study of several alkali halides and evaporated gold films indicating a reduction of the lattice constant by up to $2 \%$ for crystals of $20 \AA$ in size.

Any quantitative experimental study of the mesoscopic misfit scenario thus requires a tool which has a resolution better than the structural changes involved. Following the results predicted by theory, the changes of the interatomic distances are in the range of between 0.05 and $0.10 \AA$ at most. Therefore the aim of an experimental verification is the determination of interatomic distance changes with an accuracy in the $0.01 \AA$ regime and with sensitivity which allows probing of sub-monolayers of material. 
Surface science in general requires the accurate and reliable knowledge of the structure at the atomic scale. As compared to the number of known bulk structures the number of known nanostructures is very limited and amounts only to a few percent of the known bulk structures. [147] To considerable extent this is related to the inherent difficulty to analyze nanostructures at the atomic scale which has recently been called the "nanostructure problem". 148

Among the techniques which are both, sensitive to the low amount of material and which allow a quantitative structure analysis, low energy electron diffraction (LEED), x-ray diffraction (XRD) and extended x-ray absorption fine structure (EXAFS) are well established. Although scanning tunneling microscopy (STM) is commonly used to investigate nanostructures, its lateral resolution is not sufficient and the atomic scale structure analysis requires thorough modeling to avoid the possibility of substantial misinterpretation of the images as a result of the convolution of the electronic structure of sample and tip. However, this is hardly done (possible) in many cases.

Among the surface structure analysis tools LEED is the one by which the most structure models have been published [147, but the quantitative LEED analysis requires application of multiple scattering calculations, which significantly complicates data analysis. On the other hand for XRD single scattering theory is sufficient in most cases and it is a well established tool for the analysis of atomic structures. As far as bulk structures are concerned the analysis is, in general, carried out in an automated way by collecting bulk Bragg reflections and applying sophisticated computer programs. By contrast, in the case of nanostructures the diffraction pattern is typically characterized by a diffuse intensity distribution of the scattered intensity rather than by sharp Bragg reflections, because a well defined lattice periodicity is missing. In addition, the coherently scattered intensity is typically orders of magnitudes weaker than Bragg reflections collected from threedimensional bulk crystals.

Recent progress has been made in the XRD study of nano-crystals using coherent diffraction made possible by third generation x-ray sources [59, 149 151]. For instance, Huang et al. [59] have studied the inhomogeneous relaxations of gold nano-crystals of 3 to $5 \mathrm{~nm}$ in diameter. Bond length contraction up to $10 \%(\approx 0.2 \AA)$ was found at the outermost shell of the nano-crystal. Where focusing of the x-ray beam onto a single nano-crystal is not possible, the measurement involves averaging over an ensemble of individual islands requiring homogeneity to yield meaningful results. 151 Two-dimensional nano-islands in the size range of 1-2 nanometers, i.e. consisting of 20 to 40 atoms only which are supported by a substrate crystal 36, 49, 61, 152 are representative for such cases which will be discussed in more detail in the following sections.

EXAFS probes the local environment around an absorbing atomic species and does not rely on long range order. From this point of view, it appears as the most straightforward technique to study the atomic structure of nano-crystals. Supported nanocystals have been investigated with an emphasis on the crystal size dependent coordination number and to study the influence of static and dynamic disorder on the accurate determination of the interatomic distance. 153. In this context sample homogeneity is also an issue since EXAFS probes the local environment around each absorbing atom, which in principle is expected to be different within a nano-crystal depending on the location of the atom.

In the following sections XRD and EXAFS experiments with emphasis on the determination of the nano-island structure are discussed. At the beginning of each section, theory is presented as far as it is necessary for the understanding of the analysis and for the interpretation of the results.

\subsection{Historical Background and Theory}

Since the first experiments by Eisenberger et al. [154], $\mathrm{x}$-ray diffraction has emerged as a powerful tool to study clean and adsorbate covered crystal surfaces at the atomic scale. Several excellent comprehensive articles and reviews have been written since then [155 157] and for details the reader is referred to these reviews. In the following we concentrate on the most relevant aspects necessary for the understanding of the results discussed in this review.

The integrated intensity $[I(h k l)]$ of an x-ray reflection is proportional to the product of the absolute square of the structure factor $[F(h k l)]$ and the Laue lattice factor $[G(h k l)]$ :

$$
I(h k l) \propto|G(h k l)|^{2} \times|F(h k l)|^{2}
$$

While the structure factor contains information about the atomic positions within the unit cell, the Laue factor sums up the contribution of the $\mathrm{N}$ unit cells. This is decisive for the appearance of Bragg-reflections:

$$
G(h k l)=\sum_{n=0}^{n-1} \exp [2 i \pi \ell n] \times g(h) \times g(k),
$$

where we have explicitly considered only the summation along the z-direction (surface normal) and included the contributions along the $\mathrm{a}$ and $\mathrm{b}$ direction in the factors $g(h)$ and $g(k)$, respectively which have the same analytical form.

It is worthwhile to shortly consider the properties of this function. For $\ell$ integer, all unit cells scatter in-phase, i.e. the phase factor is equal to 1 . Secondly, for an infinite crystal corresponding by a summation from $n \in$ $(-\infty,+\infty)$, reciprocal lattice points are obtained, were the Bragg reflection is represented by the Dirac function $\delta(\ell)$ : $G(h k \ell)=\delta(\ell) \times g(h) \times g(k)$. Summation over $n \in(-\infty, 0]$ only, which is equivalent to a semi-infinite crystal with a surface at $n=0$, leads to the well known expression for 
the so called crystal truncation $\operatorname{rod}(\mathrm{CTR})$ :

$$
\begin{aligned}
F_{C T R} & =\mathrm{F}(h k \ell) \sum_{n=-\infty}^{0} e^{2 i \pi \ell n} \times g(h) \times g(k)= \\
& =\frac{F(h k \ell)}{1-e^{-i 2 \pi \ell}} \times g(h) \times g(k),
\end{aligned}
$$

which after calculation of the absolute square yields:

$$
\left|F_{C T R}\right|^{2}=\frac{|F(h k \ell)|^{2}}{4 \sin ^{2}(\pi \ell)} \times|g(h)|^{2} \times|g(k)|^{2}
$$

In consequence, the truncation of the crystal (here explicitly discussed for the c-axis only) gives rise to a continuous distribution of intensity along the reciprocal $c^{*}$ axis, i.e. $\ell$ becomes a continuous parameter. Although the appearance of the CTR has been discussed already by v. Laue in 1936 158, clear-cut experimental verification for various crystal surfaces was provided by Robinson in 1986 [159] using synchrotron radiation.

On the basis of Eq. 4 it can be verified that at the anti-phase condition $[\ell=(2 n+1) / 2$, where $n \in \mathbb{Z}]$, the total scattered amplitude is equal to $\left|F_{C T R}\right|^{2}=1 / 4$, i.e. it corresponds to $1 / 4$ of a monolayer (for a single layer of atoms in a primitive structure $\left(|\mathrm{F}(h k \ell)|^{2}=1\right)$ the relation $\left|F_{C T R}\right|^{2}=1$ holds). On the other hand, at $\ell=2 n$ corresponding to the in-phase condition Eq. 4leads to a divergence of the scattered intensity at the bulk Bragg reflections. This is because the finite penetration $(\mu)$ of the $\mathrm{x}-$ ray beam by photoabsorption and extinction is neglected. Mathematically the finite penetration can be taken into account by multiplying the denominator with the damping term $\exp [-t / \mu]$. It lifts the divergence at the Bragg reflections, but its contribution is negligible away from the bulk Bragg condition. Typically $\mu$ varies between micrometers and approximately $100 \AA$. The latter value is achieved under total external reflection conditions of the primary beam. 155] It follows that even under total reflection conditions there is no "intrinsic" surface sensitivity of XRD. Surface sensitivity solely emerges from the suitably chosen reflections in reciprocal space which are related to the presence of the surface as in the case of the CTRs. Thus surface sensitivity in XRD originates from a Fourier filtering in $k$-space. In consequence, this technique is commonly referred to as surface x-ray diffraction (SXRD).

Summarizing the preceding discussion of Eq. 4 it should be emphasized that it is the suppression of the bulk scattered intensity at positions away from the bulk Bragg reflections which allows the analysis of the surface structures, since only under this condition the scattering contributions from substrate and adsorbate are comparable in magnitude. This is of utmost importance for the case of supported nano-islands which grow epitaxially on a substrate. To clarify this condition in more detail Fig. 25 shows a schematic model of a copper crystal in side view. Along the c-axis only the two upper unit cells are shown together with an adsorbate at a position $\vec{r}$ relative to the origin of (a)

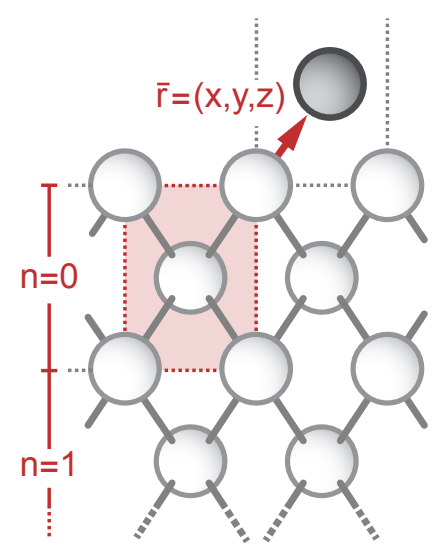

(b)

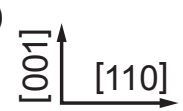

bulk unit cell

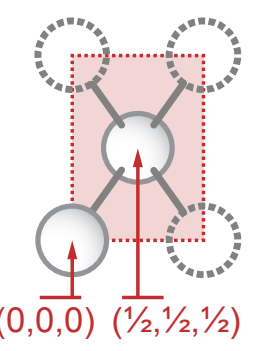

Figure 25: (a) Schematic side view ( $x-z$ plane) of a semi-infinite copper crystal where one surface unit cell is occupied by an adsorbate atom located at position $\vec{r}$ from the origin. Only one of two surface unit cells is occupied corresponding to a coverage $\theta=0.5$. For details see text. (b) The unit cell of the copper bulk containing two atoms at $(0,0,0)$ and $(1 / 2,1 / 2,1 / 2)$ according to the body centered tetragonal setting.

the topmost substrate unit cell. For the copper crystal we have chosen the bulk body centered tetragonal setting leading to the structure factor: $F(h k \ell)=f_{\mathrm{Cu}} e^{i \pi(h+k+\ell)}$ which takes into account the two copper atoms (atomic scattering factor $f_{\mathrm{Cu}}$ ) within the unit cell located at the positions $(0,0,0)$ and $(1 / 2,1 / 2,1 / 2)$. Multiplication of the structure factor with the Laue lattice factor leads to the expression for the total CTR intensity:

$$
\begin{aligned}
\left|F_{C T R}\right|^{2} & =\mid \frac{f_{\mathrm{Cu}}}{1-e^{-i \pi(h+k+\ell)}}+ \\
& +\left.\theta \cdot f_{\mathrm{Co}} \cdot e^{i 2 \pi(h x+k y+\ell z)} \cdot T(\vec{q})\right|^{2},
\end{aligned}
$$

where we have assumed that a cobalt atom with atomic scattering factor $f_{\text {Co }}$ is located on the surface of the substrate with a coverage $\theta$ at the position $(x, y, z)$ within the surface unit cell. The displacement factor $T(\vec{q})$ represents the Fourier-transform of the probability density function, $p d f(\vec{r})$, describing in full generality the displacement distribution (thermal and static) of the atoms out of the equilibrium position. For details we refer to Refs. 160, 161. The remaining parameter, $\theta_{\mathrm{Co}}$, represents the probability to find an atom at the atomic site $(x, y, z)$, i.e. in the present case it is equivalent with the coverage: $\theta_{\mathrm{Co}} \in[0,1]$.

In summary, Eq. 5 provides the basis to analyze the structure of adsorbate atoms supported by a substrate. The following section focuses on the analysis of the atomic relaxations within the cobalt nanosilands.

\subsection{SXRD analysis of the mesoscopic relaxations in Co nano-islands on $\mathrm{Cu}(001)$}

Equation 5 of the previous section provides the conceptual background for the analysis of the atomic relaxations of the cobalt atoms within the nano-islands. As previously 

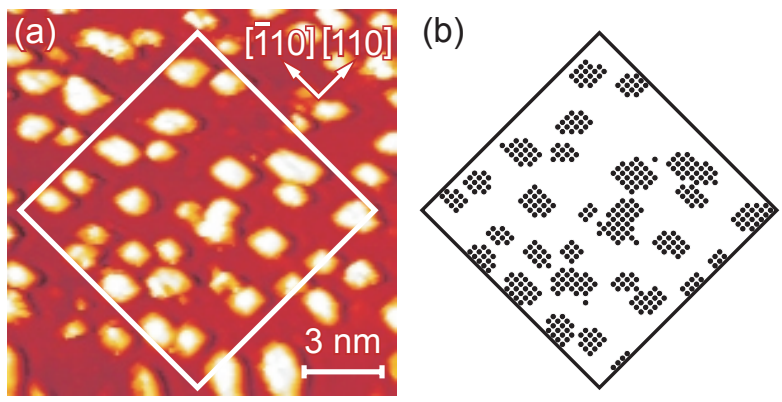

Figure 26: (a): Constant current $15 \times 15 \mathrm{~nm}^{2}$ STM image $(\mathrm{U}=0.5 \mathrm{~V}$, $\mathrm{I}=2.0 \mathrm{nA}$ ) of $0.3 \mathrm{ML}$ cobalt deposited on $\mathrm{Cu}(001)$ at $170 \mathrm{~K}$. (b): Positions of cobalt atoms derived by placing a $2.56 \times 2.56 \AA^{2}$ mesh on the within the white frame $\left(10 \times 10 \mathrm{~nm}^{2}\right)$. The unrelaxed positions with cobalt in fourfold hollow sites where used as basis for the molecular dynamic calculations to derive the relaxed positions. Figure adapted with permission from Ref. 61

discussed homogeneity of the sample is an important issue. This requirement is perfectly met in the present case where a sub-monolayer amount of cobalt atoms was deposited on a clean $\mathrm{Cu}(001)$ surface at substrate temperature of $170 \mathrm{~K}$. The low temperature was chosen to avoid intermixing as far as possible and to avoid any coalescence of cobalt islands as theory predicts an inverse correlation between island size and misfit induced atomic relaxations. 36.

Intermixing was reported to set in at $320 \mathrm{~K}$ [162] which in STM images can be detected by the bimodal island size distribution of the growing islands. 163] We rather determine a Poisson like island size distribution which according to nucleation theory is indicative for the absence of intermixing. 164, 165 .

Fig. 26(a) shows a $15 \times 15 \mathrm{~nm}^{2}$ constant current STM image of 0.3 monolayers (ML) of cobalt deposited at $170 \mathrm{~K}$. On the $\mathrm{Cu}(001)$ surface one ML corresponds to $1.53 \times$ $10^{15}$ atoms $/ \mathrm{cm}^{2}$. The islands are of monoatomic height, isometric in shape and randomly distributed. Fig. 26.(b) shows a map of the unrelaxed cobalt atomic positions (black dots) within the $10 \times 10 \mathrm{~nm}^{2}$ sized white frame in (a) which were obtained by placing a square mash of copper unit cell size $\left(2.56 \times 2.56 \AA^{2}\right)$ onto the frame. It can be seen that most islands contain $10-20$ atoms, only some are larger with up to 40 atoms. This is in good agreement with the results of an earlier SXRD profile analysis of $0.5 \mathrm{ML}$ room temperature deposited cobalt on $\mathrm{Cu}(001)$ where an island size of $24 \AA$ was found. 166

The unrelaxed (hollow site) positions of 431 cobalt atoms within the islands shown in Fig. 26. (b) where used as the basis for molecular dynamics (MD) calculations. The result of the MD calculation is presented in Fig. 27.

Misfit induced relaxations involving a contraction of the interatomic cobalt distances involve a shift of cobalt atoms out of the unrelaxed hollow sites. The position of all 431 cobalt atoms relative to the hollow site in the center $(x=y=0)$ is shown in Fig. 27(a). Fig. 27 (b) shows the distribution of the shifts projected to the [110] direction together with a superimposed Gaussian distribution
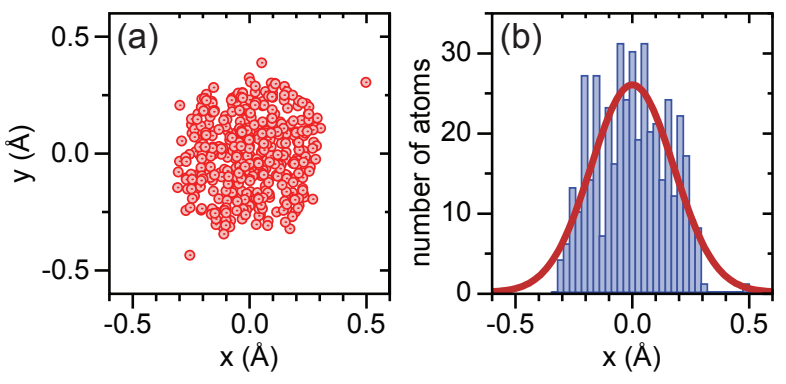

Figure 27: (a): MD calculated positions of 431 cobalt atoms within the frame (see Fig. 26 relative to the fourfold hollow position $(x=y=0)$ of the copper substrate. (b): Distribution of the atomic positions projected to the [110] direction. A Gaussian fit approximately represents the static disorder with a root mean squared amplitude of $\sqrt{\left\langle u^{2}\right\rangle}=0.18 \AA$. See Ref. 61

function. It is characterized by a root mean squared (rms) width of $\sqrt{\left\langle u^{2}\right\rangle}=0.18 \AA$ corresponding to a Debye parameter of $B=2.56 \AA^{2}$ using the relation $B=8 \pi^{2}\left\langle u^{2}\right\rangle$. This value is almost an order of magnitude larger than bulk thermal disorder at $\mathrm{T}=170 \mathrm{~K}\left(B \approx 0.3 \AA^{2}\right)$. In summary, the misfit induced relaxations emerges in a large static disorder of the adsorbate atoms which modifies the intensity scattered by the ad-layer and forms the basis of the SXRD experiments.

Following this approach and taking into account that the average adsorption site is the hollow site in the center of the unit cell $(x=y=1 / 2)$ equation 5 can be rewritten as:

$$
\begin{aligned}
\left|F_{C T R}\right|^{2} & =\mid \frac{f_{\mathrm{Cu}}}{1-e^{-i \pi(h+k+\ell)}}+ \\
& +\left.\theta_{\mathrm{Co}} f_{\mathrm{Co}} e^{i \pi(h+k)} \cdot e^{i 2 \pi \ell z} \cdot e^{-B \cdot q^{2} / 4}\right|^{2},
\end{aligned}
$$

where q represents the magnitude of the scattering vector and for the adsorbate the Debye-Waller like damping factor is added containing the Debye parameter $B$ (note, that for simplicity we have not included the damping factors for the substrate, which are considerably smaller). The experiments require high precision of the data collection since the effect of the static disorder is small and several additional parameters (e.g. z-position of the cobalt atoms, the first copper interlayer spacing and the $B$-factors of the first two copper layers) need to be refined simultaneously. The effect of the static disorder on the scattering amplitude can be estimated as follows: At the (100) antiphase condition where the surface sensitivity is at a maximum, the magnitude of the scattering vector is equal to $q=0.39 \AA^{-1}$. Using $\sqrt{\left\langle u^{2}\right\rangle}=0.18 \AA\left(B=2.56 \AA^{2}\right)$ from the MD-calculations, one obtains $T\left(q=0.39 \AA^{-1}\right)=0.90$, i.e the static displacement reduces the structure factor amplitude of the ad-layer by about $10 \%$ relative to that of the ordered structure.

Experiments were carried out at the European Synchrotron Radiation Facility (ESRF) in Grenoble (France) and at the Advanced Photon Source (APS) in Argonne 


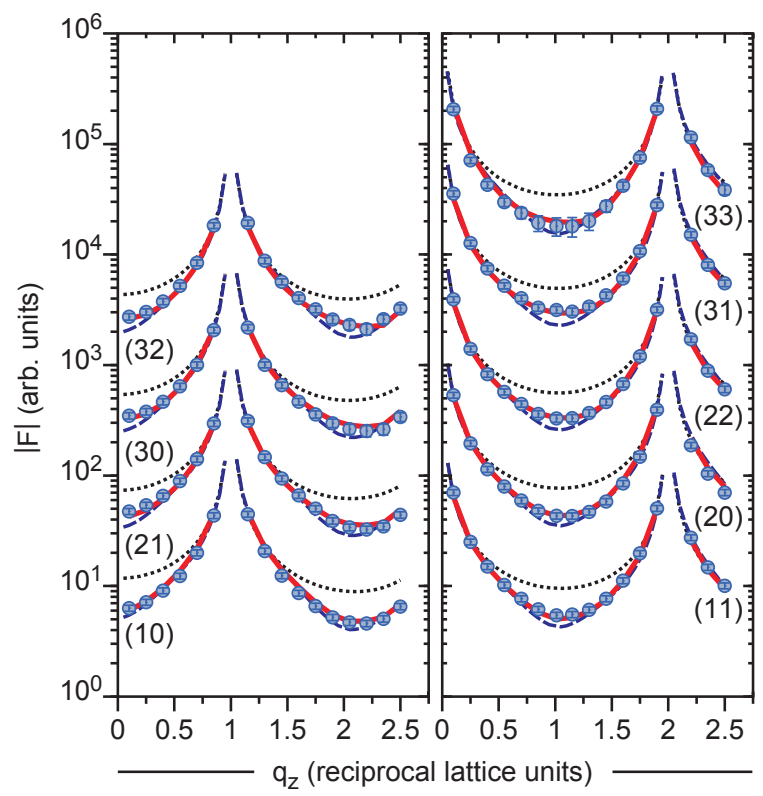

Figure 28: Experimental (symbols) and calculated structure factor amplitudes (lines) for $0.3 \mathrm{ML}$ cobalt on $\mathrm{Cu}(001)$ along nine CTRs. The black dotted line represents the calculation for the uncovered $\mathrm{Cu}(001)$ surface. The model with $0.3 \mathrm{ML}$ cobalt located in hollow sites without static disorder leads to the dashed lines which do not fit the data well near the anti-phase conditions between the bulk Bragg peaks $(h+k+\ell=2 n)$. The red line corresponds the model including static disorder of the cobalt atoms reducing their scattering amplitude. For details see text and Refs. 61152

(IL, USA). Fig. 28 shows the CTRs for $0.3 \mathrm{ML}$ cobalt on $\mathrm{Cu}(001)$. Experimental structure factor amplitudes $\left(\left|F_{C T R}\right| \propto \sqrt{I_{o b s}}\right)$ which are represented by symbols were obtained from integrated intensities $\left(I_{o b s}\right)$ and corrected for instrumental parameters as discussed in Refs. [167, 168. In general, uncertainties are calculated by the quadrature sum of the statistical and the systematic error, the latter being estimated by the equivalence of symmetry equivalent reflections (for details see Ref. 156).

Lines in Fig. 28 correspond to calculated structure factor amplitudes $\left(\left|F_{\text {calc }}\right|\right)$ along several CTRs. In general, between the bulk Bragg reflections (condition: $h+k+\ell=$ $2 n$ ) the CTRs are characterized by the U-shape like profile (in log-scale). The black dotted line represents $\left|F_{\text {calc }}\right|$ for the uncovered $\mathrm{Cu}(001)$ surface. Secondly, the dashed line corresponds to $\left|F_{\text {calc }}\right|$ assuming $0.3 \mathrm{ML}$ of cobalt to reside in hollow sites without static disorder. Close inspection indicates that especially near the anti-phase condition and at higher magnitudes of of the scattering vector [see e.g. rods $(31),(32),(33)]$ a significant deviation between $\left|F_{\text {calc }}\right|$ and $\left|F_{C T R}\right|$ is observed. It is related to the (too strong) ad-layer contribution which at the anti-phase condition is given by the second summand on the right hand side of Eq. 5. $\mid F$ calc $|=| 1 / 2 \cdot f_{\mathrm{Cu}}-\theta_{\mathrm{Co}} \cdot f_{\mathrm{Co}} \mid$. This disagreement is removed by allowing for the Debye-Waller like damping of the adsorbate scattering amplitude by multiplying with the factor $T(q)=e^{-B \cdot q^{2} / 4}$. For the accurate determination of $B$ the precise calibration of the cobalt coverage

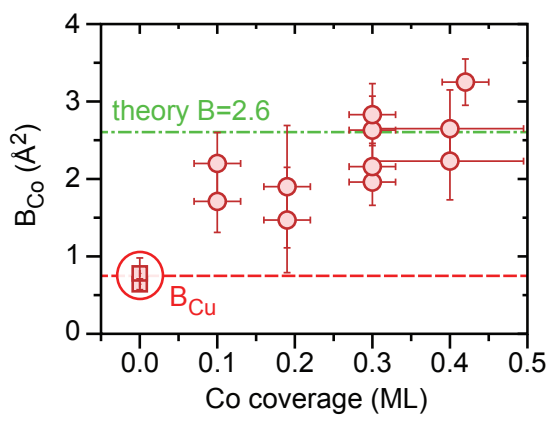

Figure 29: Debye parameters $B$ versus cobalt coverage for cobalt nano-islands on $\mathrm{Cu}(001)$. Values in the range between $B=1.5$ and $3.2 \AA^{2}$ are obtained which is in excellent agreement with $B \approx 2.6 \AA^{2}$ derived from MD calculations (horizontal line). 61

$\left(f_{\mathrm{Co}}\right)$ is mandatory as outlined in Refs. 61 and 152 .

Fig. 29 summarizes the Debye parameters for samples covered by cobalt in the 0.1 to $0.4 \mathrm{ML}$ thickness range. B-factors were obtained by fitting the calculated structure factor amplitudes to the experimental ones [61,152]. Large values between 1.5 and $3.2 \AA^{2}$ were found which agree well with $B \approx 2.6 \AA^{2}$ derived from the MD calculations (see horizontal dashed line).

Solid (red) lines in Fig. 28 correspond to the best fit obtained by this model. The fit quality is quantified by the un-weighted residual $\left(R_{u}\right)$ or by the goodness of fit (GOF) parameter. [169] While $R_{u}=\sum\left(|| F_{\text {obs }}|-| F_{\text {calc }}||\right) / \sum \mid F_{\text {obs }}$ is the normalized average relative deviation between $\left|F_{\text {calc }}\right|$ and $\left|F_{o b s}\right|$ the GOF takes into account the experimental uncertainties and the difference between the number of data-points and parameters. Excellent fits were obtained with $R_{u} \approx 4-6 \%$ and GOF $\approx 1.0$, the latter indicating that the deviation between $\left|F_{\text {calc }}\right|$ and $\left|F_{\text {obs }}\right|$ is within one standard deviation.

It should be noted that without temperature dependent measurements static and thermal disorder cannot be separated. In consequence, the $B$-factors in Fig. 29 represent both, static and dynamic disorder. However, at $\mathrm{T}=170 \mathrm{~K}$ even for atoms located at a crystal surface dynamic disorder related to thermal vibrations is at least half an order of magnitude smaller than the static disorder. For instance, the $B$-factors for the uppermost copper layers are equal to 0.47 and $0.81 \AA^{2}$ 61, 170.

In summary, the structure model taking into account the large static disorder provides compelling quantitative evidence for the presence of atomic relaxations as predicted by the MD calculations for a mesoscopically misfitted adlayer.

\subsection{Mesoscopic misfit effect on the structure in the sur- factant system $\mathrm{Fe} / \mathrm{O} / \mathrm{Fe}(001)-p(1 \times 1)$}

Atomic relaxations induced by mesoscopic misfit is a quite general phenomenon and the previous section has dealt with a simple hetero-epitaxial system where a metallic species (cobalt) was deposited on a metal substrate 
(copper). In this section we go one step further by studying a system in which a nonmetallic adsorbate is involved in a homo-epitaxial system, namely oxygen located on $\mathrm{Fe}$ nano-islands on $\mathrm{Fe}(001)$. Oxygen deposited on iron surfaces is well known to serve as a surfactant [171, 172, as it promotes layer-by-layer growth of subsequently deposited iron. Moreover, the adsorption system $\mathrm{O} / \mathrm{Fe}(001)$ is widely investigated due to its intriguing magnetic properties. Already in 1985 Huang et al. 173 discussed the correlation between the oxygen induced expansion of the top iron interlayer spacing $\left(\Delta \mathrm{d}_{12} / \mathrm{d}_{12} \approx 16 \%\right)$ [46, 174 177] with the enhancement of the surface iron magnetic moment. In the following years experimental and theoretical studies followed focusing on the enhanced magnetic moment and the increased exchange splitting [178 182, also aiming to employ oxygen covered $\mathrm{Fe}(001)$ as a spin analyzer. [183.

An ordered structure of oxygen on $\mathrm{Fe}(001)$ is easily prepared by dosing several Langmuirs $\left(\mathrm{L}, 1 \mathrm{~L}=10^{-6}\right.$ Torr $\cdot \mathrm{s}=$ $\left.1.33 \times 10^{-6} \mathrm{mbar} \cdot \mathrm{s}\right)$ of oxygen on the atomically clean iron surface 184 followed by annealing up to $500^{\circ} \mathrm{C}$. 174, 177, 182. Subsequent iron deposition follows the layer-bylayer growth mode calibrated by the oscillatory intensity variation of SXRD reflections collected at the anti-phase position along a CTR [177, 181.

Iron nano-islands of about $2 \mathrm{~nm}$ in diameter and of monoatomic height are observed by STM after stopping the iron deposition at approximately 0.5 ML coverage. The atomic structure of these nano-islands was analyzed using SXRD by collecting the integrated reflection intensities along 16 crystal truncation rods reducing to eight by symmetry equivalence on the basis of the $p 4 \mathrm{~mm}$ plane group symmetry. 46] The rapid collection of such a huge data set consisting of approximately 300 independent reflections was achieved by using a two-dimensional pixel detector. [185] It also greatly improves the accuracy of the data collection. This is reflected by the equivalence of symmetry related reflections in the range between 2 and $4 \%$.

Fig. 30(a) shows a schematic model of the surface atomic structure of $0.5 \mathrm{ML} \mathrm{Fe}$ on $\mathrm{O} / \mathrm{Fe}(001)-\mathrm{p}(1 \times 1)$. In correspondence with the constant current STM image $(\mathrm{U}=$ $-0.6 \mathrm{~V}, \mathrm{I}=100 \mathrm{pA}$ ) shown in (b), the surface can be separated into two equal fractions, namely the part of the remaining terrace labelled by "terrace" (left) and the part covered by monoatomic iron nano-islands with a diameter of approximately $20 \AA$ labelled by "rim/island" (right). A height profile along the line in (b) is shown in (c).

On the terrace $(\mathrm{t})$, oxygen atoms labelled by $O_{t}$ are located in fourfold hollow sites at a vertical distance of $\mathrm{d}_{\perp}=0.42 \pm 10 \AA$ above iron atoms labelled $\mathrm{Fe}_{\mathrm{t}}$. The first interlayer spacing between $\mathrm{Fe}_{\mathrm{t}}$ and $\mathrm{Fe}_{\mathrm{b}}$ (where $b$ stands for "bulk") equals to $\mathrm{d}_{12}=1.62 \pm 0.02 \AA$ corresponding to a $13 \%$ expansion relative to the bulk spacing of $1.43 \AA$. Within the experimental uncertainty these parameters are in good agreement with the SXRD structure analysis of the $\mathrm{O} / \mathrm{Fe}(001)-\mathrm{p}(1 \times 1)$ surface by Parihar et al. $\quad\left(\mathrm{d}_{\perp}=\right.$ $\left.0.48 \pm 0.08 \AA, \mathrm{d}_{12}=1.66 \pm 0.02 \AA\right)$. 177
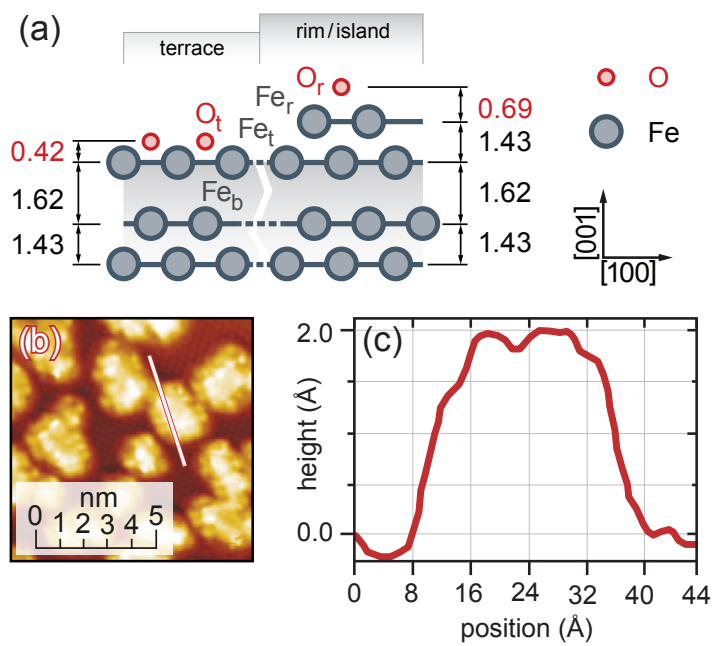

Figure 30: (a): Structure model for 0.5 ML iron dosed on $\mathrm{O} / \mathrm{Fe}(001)-\mathrm{p}(1 \times 1)$. The surface structure is composed of two parts labelled by "terrace" (left) and "rim/island" (right). The terrace structure corresponds to the $\mathrm{O} / \mathrm{Fe}(001)-\mathrm{p}(1 \times 1)$ geometry, the $\mathrm{rim} /$ island structure is related to the part of the surface which is covered by deposited iron forming nano-sized islands with oxygen atoms on top. Numbers indicate distances in $\AA$ (b): Constant current image of iron nano-islands. (c): Profile along the line in (a). Adapted with permission from Ref. 46

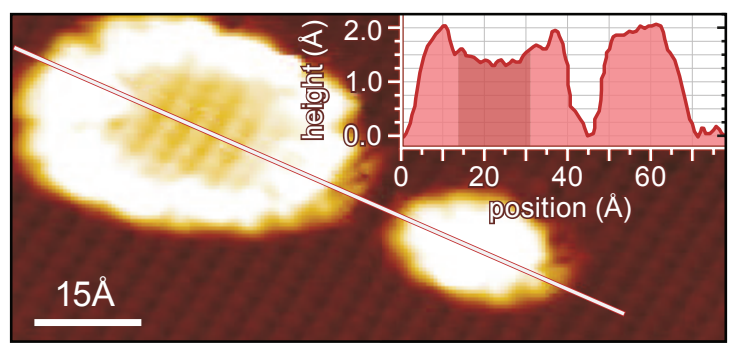

Figure 31: Constant current STM images $\left(8.0 \times 3.6 \mathrm{~nm}^{2}\right.$, sample bias $-0.2 \mathrm{~V}, \mathrm{I}=100 \mathrm{pA})$ images of two Fe islands $(\oslash \approx 20$ and $35 \AA)$. 46. The inset shows the apparent height profile along the line.

The adsorption geometry is significantly different within the rim/island $(r)$ part of the surface with atoms $\mathrm{O}_{\mathrm{r}}$ and $\mathrm{Fe}_{\mathrm{r}}$. Here, the adsorption height is $60 \%$ larger $\left(\mathrm{d}_{\perp}=\right.$ $0.69 \pm 10 \AA)$ whereas the Fe interlayer $\left(\mathrm{Fe}_{\mathrm{r}}-\mathrm{Fe}_{\mathrm{t}}\right)$ spacing is bulk like $(1.43 \AA)$ and the second one $\left(\mathrm{Fe}_{t}-\mathrm{Fe}_{b}\right)$ is enhanced. These modifications of the adsorption geometry are a consequence of the island size in the nanometer range.

A proof that a size effect plays a major role in the modification of the oxygen adsorption geometry is provided by constant current STM image in Fig. 31 reproduced from Ref. 46. It shows two Fe nano-islands with an approximate diameter of 2 and $4 \mathrm{~nm}$, respectively. In the case of the large island two regions are observed: (i) the terrace structure in the interior and (ii): an enhanced contrast and a loss of atomic resolution at the "rim" of the island (width $\approx 1 \mathrm{~nm}$ ) corresponding to an apparent elevation of $0.75 \AA$ relative to the inner part of the island whose structure is equivalent to that of a $\mathrm{O} / \mathrm{Fe}(001)-\mathrm{p}(1 \times 1)$ terrace. The small island is composed of the "rim" like structure only. 

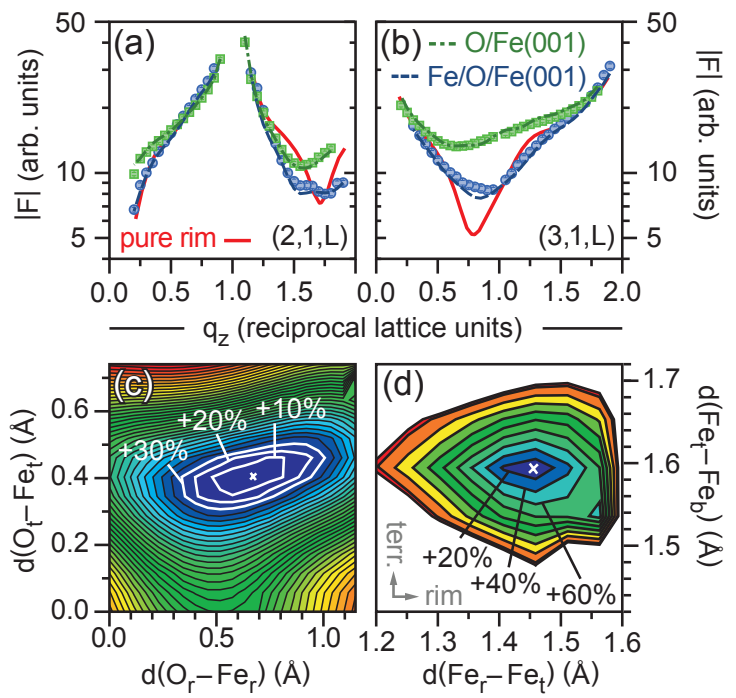

Figure 32: (a,b): Experimental (symbols) and calculated structure factor amplitudes for $\mathrm{O} / \mathrm{Fe}(001)-\mathrm{p}(1 \times 1)$ and $0.5 \mathrm{ML}$ of Fe deposited on $\mathrm{O} / \mathrm{Fe}(001)-\mathrm{p}(1 \times 1)$ as labelled. Green and blue lines represent $\left|F_{\text {calc }}\right|$ for the pure terrace structure $[\mathrm{O} / \mathrm{Fe}(001)-\mathrm{p}(1 \times 1)]$ and the structure model of Fig. [30 respectively. A pure island structure does not fit the data well (red line). (c,b): Contour plots of the goodness of fit versus the oxygen adsorption heights and the first FeFe interlayer spacings for the terrace and the island structure. The cross marks the minimum at GOF $\approx 1.2$. The contour level spacing is indicated. Based on data from Ref. 46

The height profile along the line indicates that the small island and the rim structure of the large island exhibit the same apparent height of $\approx 2 \AA$ relative to the level of the lower terrace. It can be concluded that their structure is at least very similar. Structural modifications as discussed above are limited to the rim region about $1 \mathrm{~nm}$ in width and in consequence, when the island size becomes smaller than $\approx 2 \mathrm{~nm}$ the structure of the whole island adopts the rim/island like structure sketched in Fig. 30(a). The STM image in Fig. 31(b) shows that under the experimental condition the SXRD experiments have been carried out the island size is in the $\approx 2 \mathrm{~nm}$ range only.

The SXRD analysis of the surface structure is highly accurate. For instance, symbols in Fig. 32(a,b) represent two high order CTRs $(21 \ell)$ and $(31 \ell)$ for the O/Fe(001)$\mathrm{p}(1 \times 1)$ surface before (green) and after (blue) dosing of $0.5 \mathrm{ML}$ of iron (symbols). Calculated CTRs corresponding to the pure $\mathrm{O} / \mathrm{Fe}(001)-\mathrm{p}(1 \times 1)$ terrace structure (green) and the model shown in Fig. 30(a) (blue) are represented by the solid lines. They closely follow the experimental ones indicating an excellent fit quality $\left(R_{u} \approx 4.5 \%\right.$, $\mathrm{GOF} \approx 1.2)$. Calculated structure factor amplitudes along the rods for different models do not fit the experimental ones. For instance, the red line represents $\left|F_{\text {calc }}\right|$ assuming a hypothetical pure rim/island structure, for which strong deviations from the $\left|F_{\text {obs }}\right|$ are observed corresponding to $R_{u} \approx 10 \%$ and $\mathrm{GOF}=2.4$.

Estimates for the uncertainty of the structural parameters are obtained from contour plots of the GOF versus

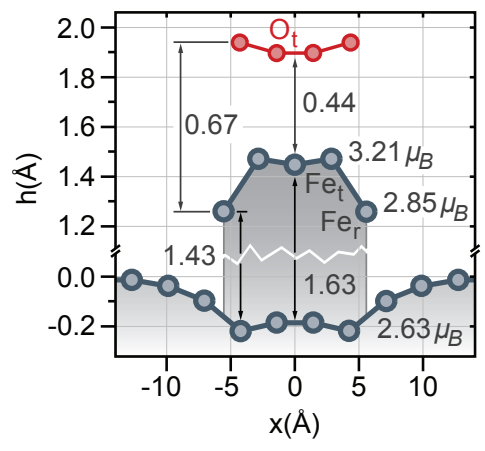

Figure 33: Calculated geometry for the rim/island and terrace structure together with calculated magnetic moments in Bohr magnetons. With permission from Ref. 46

the vertical adsorption heights of the oxygen atoms above the iron layer $\left[\mathrm{d}\left(\mathrm{O}_{\mathrm{r}}-\mathrm{Fe}_{\mathrm{r}}\right), \mathrm{d}\left(\mathrm{O}_{\mathrm{t}}-\mathrm{Fe}_{\mathrm{t}}\right)\right]$ and the first iron interlayer spacings $\left[\mathrm{d}\left(\mathrm{Fe}_{\mathrm{r}}-\mathrm{Fe}_{\mathrm{t}}\right), \mathrm{d}\left(\mathrm{Fe}_{\mathrm{t}}-\mathrm{Fe}_{\mathrm{b}}\right)\right]$ for the terrace and rim/island structure, respectively. They are shown in Fig. 32 (c) and (d). Allowing for a $10 \%$ increase of the GOF relative to the minima marked by crosses (see labels in Fig. 32 uncertainties of approximately $\pm 0.10 \AA$ and $0.03 \AA$ are derived for the oxygen/iron and iron/iron distances, respectively. 169.

The experimental structure parameters were confirmed by ab-initio calculations carried out in the framework of the density functional theory (DFT). The calculated structure parameters for the terrace and the rim/island part of the structure are outlined in Fig. 33 together with the magnetic moments in Bohr magnetons $\left(\mu_{\mathrm{B}}\right)$. The model closely resembles the large island in the STM image in Fig. 31 characterized by a terrace structure in the center $\left(\mathrm{O}_{\mathrm{t}}\right.$ and $\left.\mathrm{Fe}_{\mathrm{t}}\right)$ and the rim with atoms $\mathrm{O}_{\mathrm{r}}$ and $\mathrm{Fe}_{\mathrm{r}}$. The calculated distances are in very good agreement with the experimental ones. For instance, at the rim of the island the oxygen adsorption height is calculated to $0.67 \AA$ (experiment: $0.69 \AA$ ). Similarly, for the terrace region of the island theory derives $0.44 \AA$ versus $0.42 \AA$ in the experiment. The same applies for the first Fe-Fe distance which is expanded to $1.63 \AA$ (1.62 $\AA$ in experiment) in the terrace region while it is bulk like $(1.43 \AA)$ at the rim. Direct comparison with the height profile in Fig. 31 proves that the STM contrast is primarily related to the atomic structure. For instance, the profile indicates a maximum elevation of approximately $2 \AA$ between the rim of the island and the lower terrace of the $\mathrm{Fe}(001)$ surface, while the SXRD analysis and the calculation finds approximately $2.1 \AA$.

The calculation of the local magnetic moments allows a deeper insight into the surface magnetic properties. Previous studies using second harmonic generation (SHG) 181 and spin polarized metastable deexcitation spectroscopy (SPDMS) 178 have reported an oscillatory behavior of the surface magnetic moment versus iron coverage. Layerby-layer growth of iron periodically modifies the surface structure from one extreme (full monolayer), where only the terrace structure is present to the other where the num- 


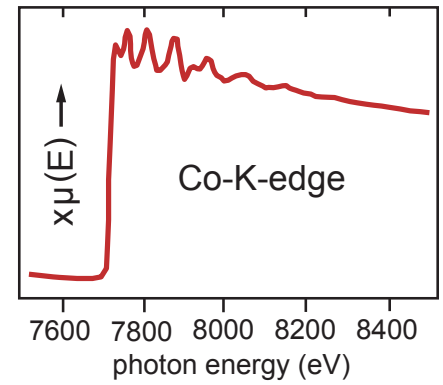

Figure 34: Linear absorption coefficient $\mu(E)$ of measured in the vicinity of the Co-K edge $\left(E_{0}=7709 \mathrm{eV}\right)$ in the transmission mode. Above the absorption edge an oscillatory modulation of $\mu(E)$ relative to the smoothly varying background $\left(\mu_{0}(E)\right)$ appears which is referred to as the extended x-ray absorption fine structure (EXAFS). For details see text.

ber of islands is at a maximum right before coalescence just as shown in Fig. 30. After completion of a monolayer the pure terrace structure is present and the surface magnetic moment $(\mathrm{m})$ is expected to be at a maximum, since for $\mathrm{Fe}_{\mathrm{t}}$ the calculations find $\mathrm{m}=3.21 \mu_{\mathrm{B}}$. On the other hand the presence of a large number of islands $(\approx 50 \%$ of the surface) reduces the average surface magnetic moment due to the presence of a large fraction of rim/island atoms $\mathrm{Fe}_{\mathrm{r}}$ which have a lower magnetic moment of $\mathrm{m}=2.85 \mu_{\mathrm{B}}$ only. On average this leads to a reduction of about $6 \%$ of the surface magnetic signal 46, 181. The structure analysis allows the conclusion that it is the finite size driven modification of the adsorption geometry is responsible for the variation of the surface magnetic properties.

\section{Experimental confirmation of mesoscopic mis- match II: Surface extended x-ray absorption fine structure}

\subsection{Historical Background and Theory}

This section briefly outlines the fundamentals of the extended x-ray absorption fine structure (EXAFS) technique as far as necessary for the understanding of the analysis of mesoscopic misfit in $\mathrm{Co} / \mathrm{Cu}(001)$. For further reading the reader is referred to excellent textbooks [186 188].

The EXAFS phenomenon, i.e the oscillatory modulation of the energy dependent linear absorption coefficient $\mu(E)$ relative to the smoothly varying background $\left[\mu_{0}(E)\right]$ is observed in the energy range several hundred electronvolt past an absorption edge (at energy $\mathrm{E}_{0}$ ). This is schematically shown in Fig. 34. The fine structure above an absorption edge is known since almost a century. The first observations were made by $\mathrm{H}$. Fricke 189 and by G. Hertz 190 and theoretically interpreted by R. de Kronig [191] involving a long range order theory. It took until the early seventies of the last century to formulate a correct theory of EXAFS in terms of a short range order [192]: It also established a path for the analysis using the Fourier Transformation (FT) of the $\chi(k)$ function which is defined as $\chi(k)=\left[\mu(E)-\mu_{0}(E)\right] / \mu_{0}(E)$, where $\mathrm{k}$ represent the

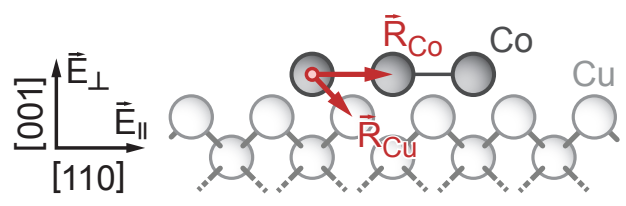

Figure 35: Idealized model of a cobalt nanostructure on $\mathrm{Cu}(001) . \mathrm{Cu}$ and Co atoms are represented by bright and dark spheres, respectively. Vectors labelled by $\vec{R}_{\mathrm{Co}}$ and $\vec{R}_{\mathrm{Cu}}$ indicate the interatomic vectors between surface Co atoms (in-plane component only) and between $\mathrm{Co}$ atoms and $\mathrm{Cu}$ atoms (out of plane). Although their magnitudes differ only by at most $0.1 \AA$, the scattering contribution can be separated by taking advantage of the polarization dependence of the EXAFS amplitude (see text and Ref. 129).

wavenumber of the electron at an energy $E$ above $E_{0}$ according to the relation: $k=\sqrt{2 m\left(E-E_{0}\right) / \hbar^{2}}$.

The function $\chi(k)$ contains contributions from different neighbor shells (indexed by $i$ ) according to the general EXAFS formula:

$\chi(k)=\sum_{i} \frac{S_{0}^{2} N_{i}^{*} F_{i}(k)}{k R_{i}^{2}} e^{\left(-2 k^{2} \sigma_{i}^{2}\right)} e^{\left(-2 R_{i} / \lambda_{i}\right)} \sin \left[2 k R_{i}+\delta_{i}(k)\right]$,

with backscattering amplitude $F_{i}(k)$, mean-free-path $\left(\lambda_{i}\right)$ and phase shift $\left[\delta_{i}(k)\right]$. The parameter $S_{0}^{2}$ describes the effect of the relaxation of the $(N-1)$ 'passive' electrons on the EXAFS amplitude after the photoelectron is emitted [188, 193]. The remaining parameters, $N_{i}^{*}$ (effective coordination number), $R_{i}$ (distance), and $\sigma_{i}^{2}$ (mean-squarerelative-displacement) are available to be fit.

In the case of K-shell EXAFS (angular momentum $\ell=0) N_{i}^{*}$ depends on the angle $\left(\alpha_{i j}\right)$ between the electric field vector $(\vec{E})$ and the interatomic vector $\vec{R}$ between absorber and backscattering atom according to:

$$
N_{i}^{*}=3 \sum_{j=1}^{N} \cos ^{2}\left(\alpha_{i j}\right)
$$

where the summation extends over all atoms $j$ of the i-th shell. Absorption from an $\mathrm{L}_{2,3}$ edge (angular momentum $\ell=1$ ) leads to a more complicated polarization dependence as discussed by Citrin 194

As an example for the application of the polarization dependence, Fig. 35 schematically shows the idealized model of a cobalt nano-island on copper. Co-atoms shown as dark spheres reside in hollow sites of the $\mathrm{Cu}(001)$ surface atoms (light spheres). 129 In this geometry the distances between Co-atoms $\left(R_{\mathrm{Co}}\right)$ and between $\mathrm{Co}$ and $\mathrm{Cu}$ atoms $\left(R_{\mathrm{Cu}}\right)$ are almost identical at $2.553 \AA$ as was shown in a LEED study by Cerda et al. [134] Owing to the mesoscopic misfit effect, $R_{\text {Co }}$ is expected to shrink by about 0.05 to $0.10 \AA$. The resulting two slightly different distances, $R_{\mathrm{Co}} \approx 2.4 \AA$ and $R_{\mathrm{Cu}} \approx 2.5 \AA$ cannot be directly resolved by the EXAFS analysis owing to the limited $\mathrm{k}$ range of the EXAFS spectrum. A beat pattern in the $\chi(k)$ function is expected according to the relation $\Delta R \approx \pi /(2 \Delta k)$. Assuming $\Delta R=0.1 \AA$, the necessary k-range is calculated 


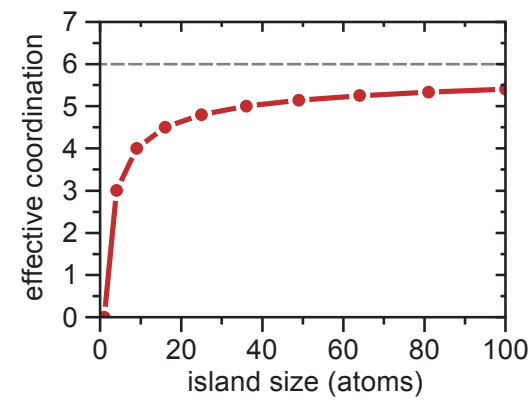

Figure 36: Average effective coordination number $\bar{N}_{\text {Co }}^{*}$ around Co atoms calculated for square Cobalt nanosislands. 129] The derivation of $\bar{N}_{\text {Co }}^{*}$ allows an estimate of the average island size.

to $\Delta \mathrm{k}=15.1 \AA^{-1}$ which is outside the experimentally accessible range of $\Delta k \approx 12 \AA^{-1}$.

Despite this limitation the unambiguous separation of the two shells $(\mathrm{i}=\mathrm{Co}, \mathrm{Cu})$ is possible by taking advantage of the polarization dependence of the effective coordination number $\left(N_{i}^{*}\right)$ according to equation (9). Two limiting cases are used: (i): Orientation of the electric field vector perpendicular to the surface plane $\left(\vec{E}_{\perp}\right)$, (ii) orientation of the electric field vector parallel to the surface plane $\left(\vec{E}_{\|}\right)$.

Under the condition $\vec{E}_{\perp}$, the nearest neighbor cobalt shell which is aligned in-plane relative to the each absorbing cobalt atom does not contribute to the EXAFS amplitude since $\alpha=90^{\circ}$ leading to $N_{\text {Co }}^{*}=0$. By contrast for $\vec{E}_{\|}$ neighboring Co atoms do contribute. Under this condition the effective coordination number varies from $N_{\mathrm{Co}}^{*}=3$ for an atom at the rim of the island to $N_{\mathrm{Co}}^{*}=6$ for an atom inside the nano-island. 129 The EXAFS analysis yields an average effective coordination number $\bar{N}_{\mathrm{Co}}^{*}$. For square islands with $n^{2}$ Co atoms $\bar{N}_{\text {Co }}^{*}$ is shown in Fig. 36 . For a typical nano-island of approximately one nanometer in diameter corresponding to about $4 \times 4=16$ atoms, the average effective coordination number is equal to $\bar{N}_{\text {Co }}^{*}=4.5$. It is significantly smaller than the limit $\bar{N}_{\mathrm{Co}}^{*}=6.0$ for an infinitely large island (see dashed line in Fig. 36). In detail, the size dependent average effective coordination number depends on the shape of the islands. However, as long as the island shape is not extremely anisotropic the approximation by a square island is a reasonably good one to estimate the island size from $\bar{N}_{\mathrm{Co}}^{*}$.

In summary, the polarization dependence of the effective coordination number allows the disentanglement of the contributions of the two nearby shells by using the following analysis strategy: (i) analysis of the EXAFS spectra collected in $\vec{E}_{\perp}$ geometry to derive $R_{\mathrm{Cu}}$ and $N_{\mathrm{Cu}}^{*}$ followed by (ii) the analysis of the EXAFS measured in the $\vec{E}_{\|}$geometry to derive $R_{\mathrm{Co}}$ and $\bar{N}_{\mathrm{Co}}^{*}$ while $R_{\mathrm{Cu}}$ and $N_{\mathrm{Cu}}^{*}$ are kept fixed. Differences between the EXAFS spectra collected for $E_{\perp}$ and $E_{\|}$are already directly observable in the $\chi(k)$ function.

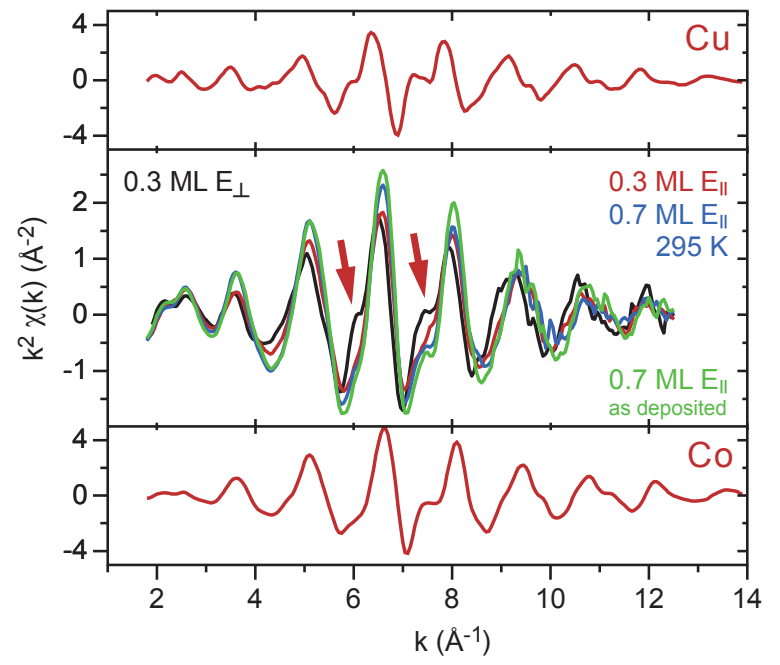

Figure 37: The $k^{2}$ weighted interference function $\chi(k) k^{2}$ for bulk $\mathrm{Cu}$ (upper panel) an Co (lowest panel) as well as for different samples: $0.3 \mathrm{ML}\left(\vec{E}_{\perp}\right)$ (black), 0.3 ML $\left(\vec{E}_{\|}\right)$(red), 0.7 ML $\left(\vec{E}_{\|}\right)$(blue), and $0.7 \mathrm{ML}\left(\vec{E}_{\|}\right)$(green). Note that the $0.7 \mathrm{ML}\left(\vec{E}_{\|}\right)$sample was measured twice, one after deposition at $160 \mathrm{~K}$ (green) and after annealing to $370 \mathrm{~K}$ followed by data collection at $295 \mathrm{~K}$ (blue).

\subsection{Experiment and data analysis}

The EXAFS measurements were carried out at $T \approx$ $160 \mathrm{~K}$ after depositing 0.3 and $0.7 \mathrm{ML}$ cobalt at $160 \mathrm{~K}$ (labelled by "LT". The modulation of the linear absorption coefficient $\mu(E)$ from which the $k^{2}$-weighted interference function is derived was measured in the fluorescence yield (FY) mode using a $\mathrm{Ge}(\mathrm{Li})$ solid state detector. The interference function of the reference bulk copper and cobalt samples were collected in the transmission mode. [129]

Fig. 37 compares the different interference functions $\chi(k) \times \mathrm{k}^{2}$ directly showing a polarization dependence. The spectrum collected for the $0.3 \mathrm{ML}$ sample in the $\vec{E}_{\perp}$ geometry resembles in several features that of the copper foil (upper panel), especially in the range at $k=5.8$ and $7.4 \AA^{-1}$ (see arrows), where a distinct shoulder is observed. This is much less pronounced in the spectra collected in the $E_{\|}$geometry. One measurement was carried out at room temperature $(295 \mathrm{~K})$ after it was prepared by $0.7 \mathrm{ML}$ deposition followed by annealing to $370 \mathrm{~K}$. It is labelled by $0.7 \mathrm{ML} E_{\|}(295 \mathrm{~K})$ and served as a test for the temperature induced coalescence of the islands and for the detection of possible anharmonic contributions to the pair distribution function, which would manifest themselves by an apparent reduction of the distances upon increasing temperature, 153 which however was not detected.

The data analysis proceeds by calculating the FT of $\chi(k) \times \mathrm{k}^{2}$ function, which was carried out by integrating from $k_{\min }=2.85 \AA^{-1}$ to $k_{\max }=11.75 \AA^{-1}$. The FT's are shown in Fig. 38 for the samples measured at $170 \mathrm{~K}$ only.

Close inspection of the peak positions shows that the peak position of the FT of the 0.3 ML sample measured in $\vec{E}_{\perp}$ geometry (black) is located at a larger $\mathrm{R}$ value 


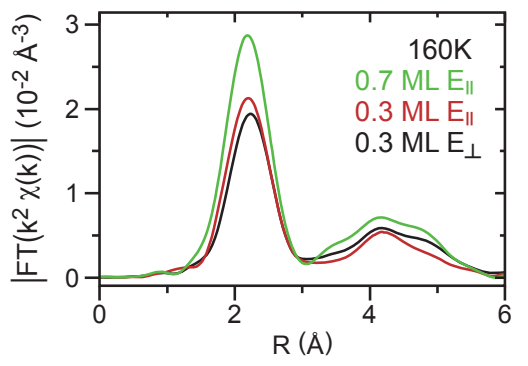

Figure 38: Fourier Transforms of the interference function of LT EXAFS spectra: $0.3 \mathrm{ML}\left(E_{\perp}\right)$ (black), $0.3 \mathrm{ML}\left(E_{\|}\right)$(red), and 0.7 ML $\left(E_{\|}\right)$(green). Note the different peak position for $0.3 \mathrm{ML}\left(E_{\|}\right)$

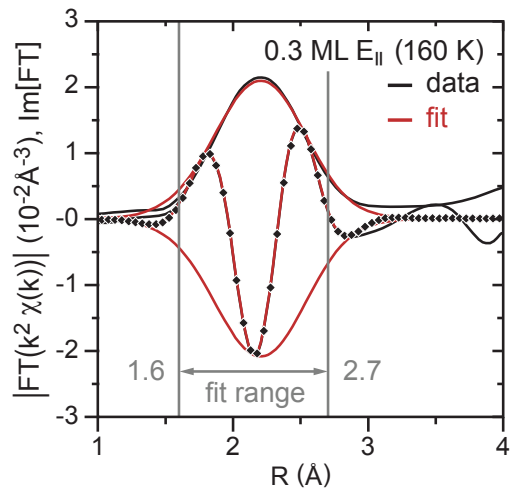

Figure 39: Experimental (black) and fitted (red) FT magnitude for the $0.3 \mathrm{ML}\left(E_{\perp}\right)$ sample together with its imaginary part of the calculated FT (inverted). Reproduced with permission from Ref. 129.

as compared to that of the $0.3 \mathrm{ML}$ and the $0.7 \mathrm{ML} \vec{E}_{\|}$ sample. Furthermore, the latter are characterized by an asymmetric line shape at the high-R side of the peak. At this point of the analysis the data analysis strategy outlined previously during the discussion of the polarization dependence of the effective coordination number becomes evident: the $\mathrm{FT}$ of the $0.3 \mathrm{ML}\left(\vec{E}_{\perp}\right)$ spectrum is related to only one (copper) shell, while the spectra related to the $\vec{E}_{\|}$geometry originate from two nearby shells (cobalt and copper). Finally, from the considerably larger amplitude of the $0.7 \mathrm{ML}\left(\vec{E}_{\|}\right)$FT it can be concluded that the effective coordination number for this sample is higher than that of the $0.3 \mathrm{ML}$ sample, which is attributed to the larger island size of the $0.7 \mathrm{ML}$ sample.

The quantitative analysis is carried out in $R$-space by fitting the FT's. The fit uses model amplitude functions $[F(k)]$ and phases derived from the FEFF7 program [195] which are implemented in the WinXAS program. [196] One example is shown in Fig. 39 for the 0.3 ML sample measured in $\vec{E}_{\perp}$ geometry. The solid black curve represents the experimental (magnitude of the) FT, while the red curves correspond to the calculated FT, and its imaginary part $\operatorname{Im}[\mathrm{FT}]$, the latter being highlighted by symbols. The $k$-range for the fit was selected to lie in the range between 1.6 and $2.7 \AA^{-1}$ avoiding contributions from higher shells.

It can be seen that the experimental and calculated

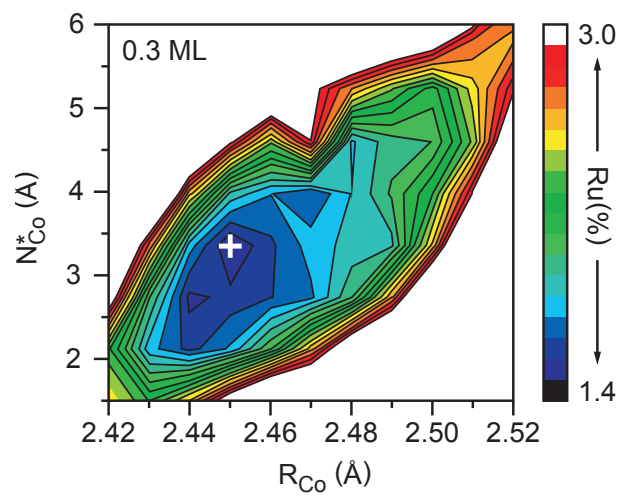

Figure 40: Contour plot of the residual versus $R_{\mathrm{Co}}$ and $\bar{N}_{\mathrm{Co}}^{*}$ for $0.3 \mathrm{ML}$ sample. The white cross corresponds to the global minimum at $R_{\mathrm{Co}}=2.45 \AA$ and $\bar{N}_{\mathrm{Co}}^{*}=3.2$. The significantly reduced value for $R_{\mathrm{Co}}$ as compared to the bulk Co-Co distance $(2.51 \AA)$ is in agreement with theoretical predictions for mesoscopic misfit induced relaxations.

FT nearly overlap. The fit quality is quantified by the unweighted residual $\left(R_{u}\right)$ which is defined as $R_{u}=\sum\left[\left(Y_{o b s}-\right.\right.$ $\left.\left.Y_{\text {calc }}\right)\right] / \sum Y_{\text {obs }}$, where $Y_{\text {obs }}$ and $Y_{\text {calc }}$ correspond to the experimental and calculated values of the FT, and the summation runs over all points within the selected $k$-range. In summary, two remarkable results were obtained: firstly, there is unambiguous evidence for the reduction of the interatomic cobalt distance from $\mathrm{R}_{C o}=2.51 \AA$ in the bulk to $\mathrm{R}^{C o}=2.45 \pm 0.02 \AA$ in the nanoisland. Secondly, the effective (average) coordination number is reduced from $\bar{N}_{\text {Co }}^{*}=6.0$ (for an infinitely large island) to $\bar{N}_{\text {Co }}^{*}=3.2$. These results are in good agreement with theoretical predictions and can be viewed as a direct proofs for the mesoscopic misfit induced structural relaxations.

Estimates of the uncertainty lie in the range of $\Delta R_{C o}=$ $0.02 \AA$ for $R_{\text {Co }}$ and $\Delta \bar{N}^{*}=0.05-0.07$. In Fig. $40 R_{u}$ is plotted versus $R_{\mathrm{Co}}$ and $\bar{N}_{\mathrm{Co}}^{*}$. The white cross indicates the global minimum at $R_{\mathrm{Co}}=2.45 \AA$ and $\bar{N}_{\mathrm{Co}}^{*}=3.2$. Allowance for an increase of $R_{u}$ in the $10-20 \%$ range leads to the estimated uncertainties for $R_{C o}$ and $\bar{N}_{\text {Co }}^{*}$.

\subsection{Summary of the results}

The previous sections have shortly outlined the most important concepts of the EXAFS data analysis applied to the verification of the mesoscopic misfit induced bond distance contraction. This section summarizes the results by comparison between experimental results and theoretical predictions. Table 1 provides an overview. Only the main structural parameters are listed, for more details we refer to Ref. 129

As reference copper and cobalt foils were used. Known parameters for crystalline copper and cobalt are listed in the first and third row. Experimentally obtained distances (second and fourth row) are very close to the expected ones. The coordination numbers are kept fixed at $\bar{N}^{*}=12$ (as indicated by the brackets). The second panel lists the parameters related to three model islands of differ- 
Table 1: Summary of the results of the EXAFS analysis. Only structural parameters $\left(R\right.$ - interatomic distance, $N^{*}$ - effective coordination number) are listed. Subscripts "Co" and "Cu" correspond to coordination shells composed of cobalt and copper atoms, respectively. Parameters for bulk copper and cobalt used as references are listed in the upper panel. The center panel lists theoretical values for idealized model islands of different size. The lower panel lists all results obtained for the ultra thin film samples. Parameters in brackets were kept fixed. Symbols $(\|)$ and $(\perp)$ refer to experimental geometry with the electric field vector parallel or perpendicular to the surface plane, respectively. Experiments were carried out at room temperature (RT) and $160 \mathrm{~K}(\mathrm{LT})$.

\begin{tabular}{l|c|c|c|c}
\hline Sample & $\mathrm{N}_{\mathrm{Cu}}^{*}$ & $R_{\mathrm{Cu}}(\AA)$ & $N_{\mathrm{Co}}^{*}$ & $R_{\mathrm{Co}}(\AA)$ \\
\hline Cu cryst. RT & 12 & 2.556 & & \\
Cu foil RT & {$[12]$} & $2.54(5)$ & & \\
Co cryst. RT & & & 12 & 2.507 \\
Co foil RT & & & {$[12]$} & $2.493(5)$ \\
\hline$\infty$ lay RT & \multirow{2}{*}{$6^{(\perp)}$} & & $6.0^{(\|)}$ & \\
$4 \times 4$ isl RT & $3^{(\|)}$ & 2.553 & $4.5^{(\|)}$ & 2.554 \\
$6 \times 6$ isl RT & & & $5.0^{(\|)}$ & \\
\hline 0.3 ML LT $E_{\perp}$ & 7.3 & 2.544 & & \\
& $(1.0)$ & $(10)$ & & \\
\hline 0.3 ML LT $E_{\|}$ & & & $3.2(5)$ & $2.45(2)$ \\
0.7ML LT $E_{\|}$ & {$[4.95]$} & {$[2.544]$} & $5.0(7)$ & $2.46(2)$ \\
0.7 ML RT $E_{\|}$ & & & $5.5(5)$ & $2.46(2)$ \\
\hline
\end{tabular}

ent size for both, the $\vec{E}_{\perp}$ and the $\vec{E}_{\|}$geometry. Distances $R_{\mathrm{Cu}}$ and $R_{\mathrm{Co}}$ correspond to the fourfold hollow adsorption site geometry according to the LEED analysis of Cerda et al. 134] The lower two panels list the fit results for the ultra thin film samples.

For the $\vec{E}_{\perp}$ ) geometry probing only the nearest copper shell, $R_{\mathrm{Cu}}=2.544 \AA$ and $N_{\mathrm{Cu}}^{*}=7.3 \pm 1.0$ was found. The latter comes unexpected since it is larger than the maximum of $N_{\mathrm{Cu}}^{*}=6.0$ for the model structures in $\vec{E}_{\perp}$ geometry (see second panel). The increase of $N_{\mathrm{Cu}}^{*}$ is attributed to intermixing $(\approx 20 \%)$ in which cobalt atoms are embedded into the copper substrate thereby increasing the effective coordination number to 12 . 129] In the following the structure parameters $\mathrm{R}_{C u}$ and $N_{\mathrm{Cu}}^{*}$ are kept fixed for the analysis of the data collected under $\vec{E}_{\|}$geometry. It should be mentioned that $N_{\mathrm{Cu}}^{*}$ has to be recalculated when changing from $\vec{E}_{\perp}$ (7.3) to $\vec{E}_{\|}$(4.95).

Structure parameters of the cobalt shell are listed on the right of the lowest panel for three samples. They indicate the reduced interatomic cobalt distances and coordination number characteristic for the mesoscopically misfitted islands. While $\mathrm{R}_{\text {Co }}$ does not depend on the cobalt coverage, the effective coordination number increases from 3.2 for the 0.3 ML sample to 5.0-5.5 for the 0.7 ML samples. Simply this can be attributed to an increased island size resulting from coalescence. 129]

\section{Experimental evidence for mesoscopic mismatch III: Monolayer-stress oscillations in hetero-epitaxy}

The epitaxial growth of a film on a substrate leads in general to an epitaxial misfit $\eta$, which is defined as the difference between the nearest neighbor atomic distances in the substrate $a_{\mathrm{s}}$ and film $a_{\mathrm{f}}$, where the respective value of the film bulk material serves as the reference, $\eta=\left(a_{\mathrm{s}}-\right.$ $\left.a_{\mathrm{f}}\right) / a_{\mathrm{f}}$. This epitaxial misfit gives rise to a biaxial stress $\tau$ in the film, which is calculated as $\tau=\frac{Y_{\mathrm{f}}}{1-\nu_{\mathrm{f}}} \eta$ for isotropic strain with the Young module $Y$ and the Poisson ratio $\nu$. The biaxial modulus of the film needs to be calculated for the given epitaxial orientation. For a cubic (001) film it is given by $Y /(1-\nu)=1 /\left(s_{11}-s_{12}\right)$, where the $s_{i j}$ are the elastic compliances of the bulk film material [10, 138, 197, 198.

One might rightfully wonder whether the application of bulk elastic properties to describe film stress in the monolayer regime is physically sound. Our empirical observation of a favorable agreement between calculated and measured film stress, for a description of the respective experiment see Section 6.1 below, indicates that bulk elasticity is indeed a viable reference in the monolayer range for films thicker than 1-2 layers for a wide selection of filmsubstrate composites. In thinner films, the film-induced surface stress change of the substrate material may contribute significantly to the measured stress [136, 198 201, and this complicates a clear cut discussion of film stress in terms of epitaxial misfit. Also, intermixing at the film substrate interface and surface alloy formation may contribute to the measured stress change in some cases [77, 202]. In cases where the epitaxial misfit is of order $10 \%$, the epitaxial order might change from a pseudomorphic growth to a growth characterized by structural relaxation already in the second layer. A prototypical example is the growth of Fe on W(110). Here, the resulting effective misfit in films thicker than 2 layers is considerably reduced [73, 203. In cases where the film material can grow in different modifications, e.g. $f c c-\mathrm{Fe}$ and $b c c-\mathrm{Fe}$, it is a priori not clear, which modificationthe stress curve varies, and this apparent should be taken as the film reference state. Here, quantitive stress measurements can resolve any ambiguity. 204 In conclusion, film stress can be reliable calculated in a few layer thin film of a definite epitaxial order. In the single monolayer regime surface stress changes and intermixing may have to be considered also.

To appreciate the magnitude of epitaxial misfit stress we point out that the biaxial modulus of metallic systems is of order of $100-200 \mathrm{GPa}$, and thus already a misfit strain of the order of $1 \%$ induces a stress of order several GPa. This is an remarkably large value, which marks the elasticity limit of any bulk material. However, in epitaxial monolayers stress of this and larger magnitude are measured, as discussed below. The corresponding elastic energy scales quadratically with lattice strain [10], $f_{\text {elast }}=Y \eta^{2} /(1-\nu)$, and it can be as large as $0.1 \mathrm{eV}$ per atom. This is a significant contribution to the total energy of the system. 
It is $3-5$ orders of magnitude larger than the magnetic anisotropy energy density, and $1-2$ orders of magnitude smaller than typical bonding energies.

A stress of this magnitude leads to sizable atomic displacements at the boundary of nanostructures, such as epitaxial island at incomplete layer coverage. As a consequence of uncompensated forces at the island boundaries, film atoms are shifted out of the pseudomorphic positions, substrate atoms are dragged along, and a complicated 3Dstructural distortion evolves [36, 41, 43, 61, 130, 205. Its spatial extent is of the order of several $\mathrm{nm}$. Thus, in $\mathrm{nm}$ small islands, this structural relaxation may be dominant for the resulting atomic structure and the physical properties, such as stress and electronic structure. In Section 6.2 below we present stress measurements which clearly show the resulting variation of film stress with a monolayer period, where film stress is reduced for islands at partial layer filling. In general, a spatial variation of the electronic structure within a single island evolves, and this aspect and its implication for the spin-dependent electronic structure and magnetism of Co bilayer islands has been discussed above in Sections 3.7 and 3.8

In the following we describe an optical deflection measurement, which we use to measure stress during film growth from the stress-induced curvature of a thin single crystalline substrate.

\subsection{Measurement of film stress with sub-monolayer sen- sitivity}

The idea to measure stress in thin films from the stressinduced curvature of a thin substrate has been first presented by Stoney some 95 years ago. 206] The measurement of adsorbate-induced surface stress and of film stress by the crystal curvature technique, as schematically indicated in Fig. 41, has developed ever since into a reliable source of quantitative data on stress with an astonishingly high sensitivity in the sub-monolayer coverage regime [10, 135, 201, 207 209]. The high sensitivity of the measurement scheme is evident from its successful application also to the measurement of magneto elastic stress in monolayers [10, 68, 209, 210, where the magnitude of stress is roughly 3 orders of magnitude smaller as compared to stress due to epitaxial misfit in the percent range.

The choice of substrates with a sufficiently large lengthto-width ratio of larger 3 , and the proper treatment of elastic anisotropy [212, 213] ensures highly accurate measurements of stress with an error bar in the low percent range. For the case of biaxial stress as induced by a pseudomorphically strained film on a single crystalline substrate the relation between stress-induced curvature change $\Delta 1 / R$ and change of stress $\Delta \tau$ is given by

$$
\Delta \tau=\Delta\left(\tau_{\mathrm{f}} t_{\mathrm{f}}\right)=\frac{Y_{\mathrm{s}} t_{\mathrm{s}}^{2}}{6\left(1-\nu_{\mathrm{s}}\right)} \Delta(1 / R),
$$

where the subscripts $\mathrm{f}$ and $\mathrm{s}$ indicate film and substrate properties, respectively. The film thickness is given by $t_{\mathrm{f}}$,

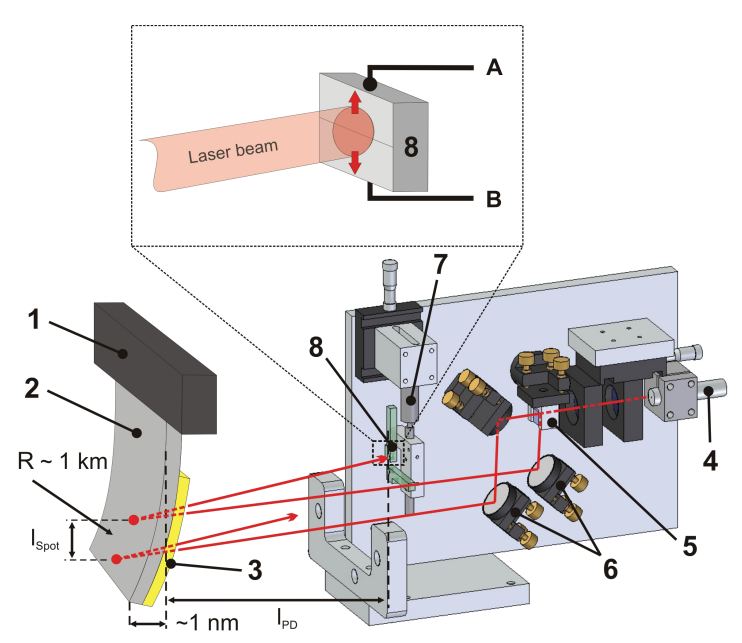

Figure 41: Schematic of the optical stress measurement setup. The sample holder (1) is located inside of the ultrahigh vacuum chamber, and it clamps a $12 \mathrm{~mm}$ long, $2.5 \mathrm{~mm}$ wide, $0.1 \mathrm{~mm}$ thin single crystal substrate (2) along its width at the top end. The film (3) is deposited onto the front surface, whereas the backside remains pristine. Film stress induces a curvature with a typical radius of curvature $R \approx$ $1 \mathrm{~km}$ for a monolayer coverage, and the bottom end of the crystal deflects by about $1 \mathrm{~nm}$. This curvature is detected by reflecting two laser beams, which are produced by a laser diode (4) and a beamsplitter (5) and directed to the sample with two mirrors (6), onto two position sensitive detectors (8). The detectors are split photodiodes, as shown in the inset, and the laser spot deflection is given by the signal $(A-B) /(A+B)$. The detectors are mounted onto a piezo translational stage $(7)$, to calibrate the deflection signal with a calibrated detector movement. The optical deflection setup is mounted outside of the vacuum chamber, and bolted directly to a window flange at a distance $l_{\mathrm{PD}} \approx 280 \mathrm{~mm}$ from the substrate. See Ref. 211 for further details. 
the substrate thickness by $t_{\mathrm{s}}$. The change of curvature $\Delta(1 / R)$ is obtained from the respective change of the the position signals $\Delta$ pos at the two position sensitive detectors

$$
\Delta(1 / R)=\frac{\Delta \operatorname{pos}_{\mathrm{top}}-\Delta \operatorname{pos}_{\mathrm{bottom}}}{2 l_{\mathrm{PD}} l_{\mathrm{Spot}}},
$$

where the laser beam separation on the film surface is given by $l_{\text {Spot }}$, as indicated in Fig. 41 .

A constant film stress induces a curvature, which increases in proportion to the film thickness. Thus, the slope of the curvature as a function of film thickness gives the film stress $\tau_{\mathrm{f}}$. In the following we show, that the slope of the stress curve is not constant. Rather, we observe a monolayer oscillation around an average film stress, which is given by the epitaxial misfit. The stress is maximal for filled layers.

\subsection{Stress oscillations with monolayer periodicity in Co layers on $\mathrm{Cu}(001)$}

The growth of $\mathrm{Co}$ on $\mathrm{Cu}(001)$ has been intensively studied, and it can be viewed as an ideal representation of layer-by-layer growth above $2 \mathrm{ML}$ deposition [30, 214, 215.

Epitaxial growth of $\mathrm{Co}$ on $\mathrm{Cu}(001)$ at $300 \mathrm{~K}$ follows the layer-by-layer mode by island nucleation on terraces of the substrate, where the islands grow in lateral size until they coalesce, and the growing layer is filled. Ongoing deposition may lead to the same processes in the next layer. Thus, as a function of film deposition, the surface morphology changes periodically from flat (clean substrate or filled layer) to rough (partial layer filling) to flat (completed layer) again with monolayer period. This periodic variation of the film roughness can be imaged directly by STM, and it can be followed in situ by reflecting an electron beam under grazing incidence from the surface, a technique known as medium energy electron diffraction (MEED) and reflection high energy electron diffraction (RHEED), depending on the electron energy. [216] A high reflected intensity indicates a flat film, whereas a rough surface leads to a reduced intensity.

The epitaxial misfit between fcc-Co $\left(a_{f c c-\mathrm{Co}}=3.548 \AA\right.$ 134] ) and $\mathrm{Cu}\left(a_{\mathrm{Cu}}=3.615 \AA\right)$ of $+1.7 \%$ gives rise to a tensile stress in the Co film. Thus, the atoms in the Co film adopt a larger Co-Co bond length as compared to the fcc-Co reference state. Diffraction experiments [30, 129] confirm that complete layers of Co grow in pseudomorphic positions on the $\mathrm{Cu}(001)$ substrate, and the expectation of an epitaxial misfit induced tensile film stress is well justified. However, for incomplete layer filling nonpseudomorphic Co sites have been identified [61, and they are ascribed to structural relaxation within nm small Co islands. This structural relaxation leads to a smaller stress in the islands, as discussed below.

The combination of stress measurement by the curvature technique with electron reflection under grazing incidence is of paramount importance to reliably correlate stress and layer filling. Corresponding experiments have

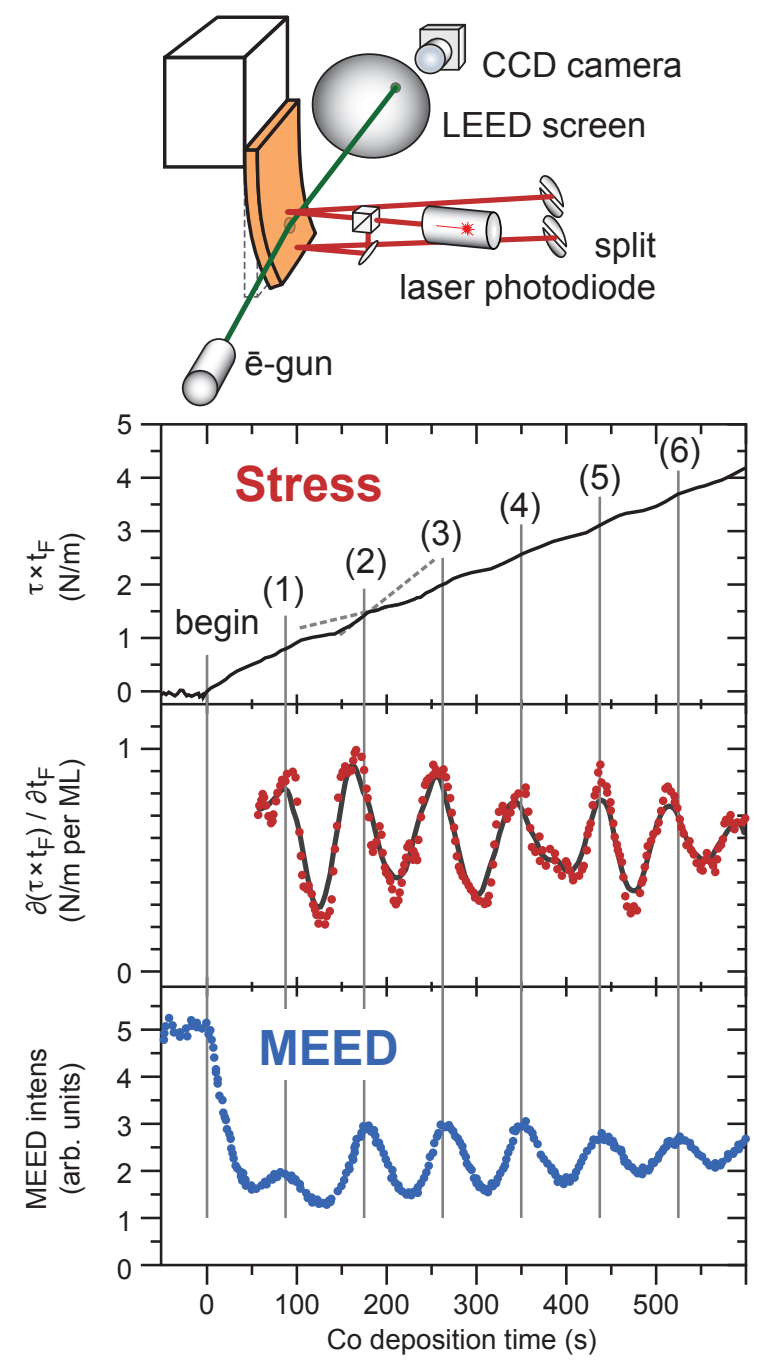

Figure 42: Simultaneous stress and MEED measurements identify monolayer stress oscillations in Co layers, during deposition on $\mathrm{Cu}(001)$ at $300 \mathrm{~K}$. The top schematic indicates the combination of the two beam curvature measurement, detailed in Fig. 41 with an in situ measurement of the sample roughness by reflecting a medium energy electron beam ( $3 \mathrm{keV}$ ) from an Auger electron spectrometer electron gun onto a LEED screen (MEED). The CCD camera monitors the intensity of the reflected electron beam. The stress measurements of the top panel exhibit tensile stress, which is ascribed to the epitaxial misfit. The plot of the slope of the stress curve in the center panel identifies monolayer stress oscillations. These stress oscillations are in phase with monolayer oscillations of the MEED intensity. 

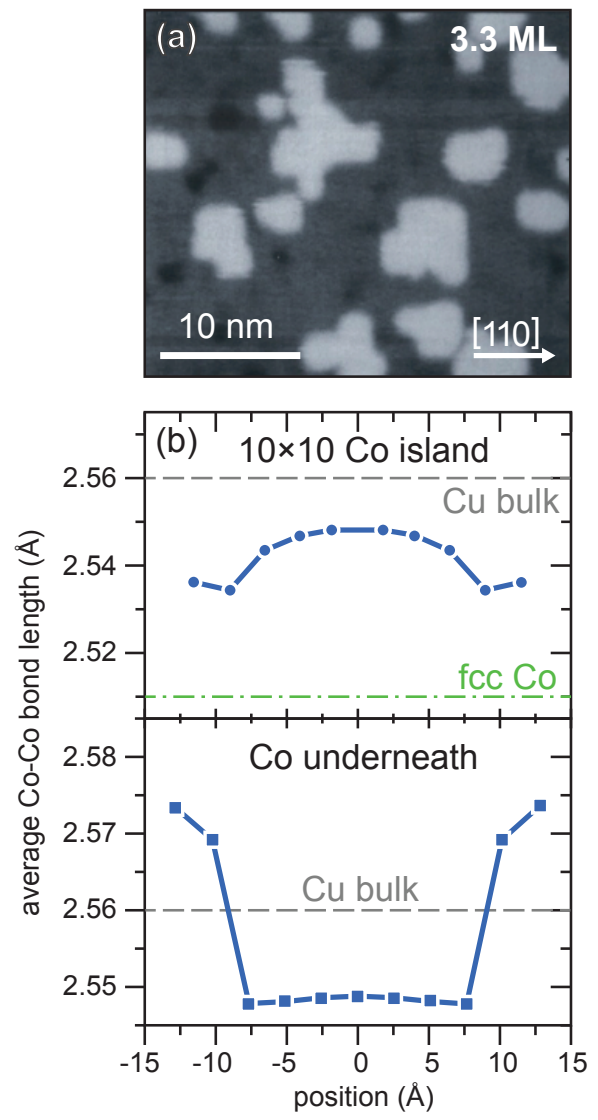

Figure 43: (a) STM constant current image of 3.3 ML Co on $\mathrm{Cu}(001)$. 214 The STM image reveals monolayer high Co islands of the fourth layer on top of the almost filled third layer. Black patches reveal openings to the second layer. Note that the Co islands have a small edge length of order $2-5 \mathrm{~nm}$, where edges are predominantly oriented along [110]. (b) Calculated Co-Co bond length of a Co island (top panel) and the Co layer underneath (bottom panel). 197. The calculations reveal a bond length in the Co island smaller than that expected for pseudomorphic growth, as indicated by all data points below the $\mathrm{Cu}$-bulk reference as a dashed line. The Co layer underneath shows a bond length which varies with position.

been performed before for semiconductor growth of III- $\mathrm{V}$ compounds 217, and we applied the technique to metalon-metal epitaxy of monolayers [128, 136, 197, 201, 202. The top panel of Fig. 42 shows a schematic of the experiment, where we use the in-line arrangement of an Auger electron spectrometer (AES) and a low energy electron diffraction (LEED) screen of the ultrahigh vacuum chamber as electron gun and electron detector, respectively.

The stress measurement in the top panel of Fig. 42 shows a positive stress change upon Co deposition. The quantitative analysis reveals an average stress increase per $\mathrm{ML}$ of $+0.6 \mathrm{~N} / \mathrm{m}(+3.4 \mathrm{GPa})$. This stress agrees favorably with the calculated misfit induced film stress of $+3.2 \mathrm{GPa}$, and this identifies epitaxial misfit as the dominant source of film stress. 197 However, a closer look reveals that the slope of the stress curve varies, which is apparent from the plot of the slope of the stress curve in the center panel. Clear variations of the slope with monolayer period are detected. The simultaneously taken MEED intensity data shown in the bottom panel identify an in-phase behavior of MEED and stress oscillations. The slope of the stress is maximum for filled layers, and it is reduced for incomplete layer filling.

We characterize the film morphology during film growth a different coverages by STM [61, 214. An example is shown in Fig. 43(a). We see that an incomplete layer filling at covereages of $3.3 \mathrm{ML}$ is linked to the growth of $\mathrm{nm}$ small monolayer Co islands on the almost filled second layer. We can already conclude that these small Co islands exert a much smaller stress on the substrate as expected from the misfit argument. But how is this possible?

The answer is given by the calculations presented in Fig. 43(b). Our calculations reveal, that although the system $\mathrm{Co}$ on $\mathrm{Cu}(001)$ is loosely described by pseudomorphic growth, at incomplete layer filling non-pseudomorphic bond sites are observed. The plots indicate that nowhere in a nm small island is the naively expected pseudomorphic $\mathrm{Co}-\mathrm{Co}$ bond length calculated. The Co-Co bond lengths in the island are considerably reduced. Note, that in the Co layer underneath also larger $\mathrm{Co}-\mathrm{Co}$ bond lengths are observed near the rim of the island. Thus, a complex structural relaxation needs to be considered on the nm scale. This disqualifies the application of epitaxial misfit to properly characterize the atomic structure in islands. [36] We conclude that the reduced Co-Co bond length in islands is the reason for the reduced film stress at incomplete layer filling. 197.

Stress measurements identify structural relaxations by the corresponding change of film stress. Its quantitative analysis goes beyond the concept of strain and elasticity, as established for continuum systems. The underlying theoretical description is demanding, as a large number of atoms in sites of reduced symmetry and their long range elastic interaction needs to be treated self consistently. Our stress measurements provide reference data as a benchmark for theory.

\subsection{Stress oscillations in other systems}

Measurements of stress oscillations in nanoscale system due to structural relaxation are extremely scarce. Our lab focussed on stress oscillations in metallic systems, and the examples of $\mathrm{Co}$ on $\mathrm{Cu}(001)$ and FeMn on $\mathrm{Cu}(001)$ are discussed in this review. We mention that also in semiconductor systems stress oscillations with monolayer period have been observed for the growth of III-V compounds. 217] The authors report periodic variations of the deflection of a GaAs cantilever during MBE growth of GaAs. The authors ascribe the RHEED and stress oscillations to variations of the step density on the surface with monolayer period due to island growth and coalescence. In addition to structural relaxation also electron quantum confinement has been proposed to cause periodic stress oscillations. 218] The authors report stress oscillations of order $100 \mathrm{MPa}$ during the growth of $\mathrm{Al}$ on a $\mathrm{Si}(111)$ cantilever. They ascribe these oscillations with a periodicity 
of twice the Fermi wavelength of $\mathrm{Al}$, around $10 \mathrm{ML}$, to a signature of electron confinement along the film thickness. Here, morphological effects can be excluded as the growth occurs in the step flow mode.

\subsubsection{Stress oscillations in FeMn monolayers on Cu(001)}

The epitaxial growth of multi-component films is a topic of considerable interest, specifically in the field of magnetism. Here, alloys are commonly used to tune the magnetic properties of atomic layers. The co-deposition of the constituents of the envisioned material on the substrate may lead to unexpected structural and compositional deviations from the expected behavior, due to possible pronounced differences of the interaction of the deposited materials with the substrate. We discuss here the growth of FeMn on $\mathrm{Cu}(001)$. 202] FeMn is a antiferromagnetic alloy, which is frequently used in nano magnetism in applications such as spin-valves. However, its magnetic properties depend sensitively on the exact stoichiometry. In this respect, the proximity between $\mathrm{Mn}$ and $\mathrm{Cu}$ may challenge a reliable adjustment of the stoichiometry. The reason is that $\mathrm{Mn}$ forms a surface alloy with $\mathrm{Cu}$ under common growth conditions. [77]

We monitor both film stress and surface roughness during co-deposition of $\mathrm{Fe}$ and $\mathrm{Mn}$ onto $\mathrm{Cu}(001)$ at $300 \mathrm{~K}$, by combined curvature and MEED measurements, as shown in Fig. 44. The stress curve shows a compressive stress change, where the initial stress drops sharply to $-0.8 \mathrm{~N} / \mathrm{m}$ at the deposition of $1 \mathrm{ML}$ and it changes to slight monolayer oscillations on a compressive stress with ongoing deposition for films thicker than $5 \mathrm{ML}$. The MEED intensity shows a sharp decay up to $5 \mathrm{ML}$, and then clear monolayer oscillations are observed in thicker films up to $18 \mathrm{ML}$, where the deposition was stopped.

The observation of a compressive stress change for FeMn growth on $\mathrm{Cu}(001)$ for films thicker than $5 \mathrm{ML}$ is in qualitative agreement with the calculated misfit of $-0.4 \%\left(a_{\mathrm{FeMn}}=\right.$ $3.629 \AA$ ). [77 However, the stress curve shows a complex dependence with thickness in the first 5 layers. As discussed before [77, we ascribe the initial sharp stress change to the formation of the $\mathrm{MnCu}$ surface alloy. In thicker films however, the FeMn alloy starts to form, and the observed stress change is ascribed to the epitaxial misfit between FeMn and $\mathrm{Cu}$.

The stress oscillations indicate a maximum of the compressive stress (most negative stress value) at filled layers (maxima of MEED signal). This behavior is at first sight different from the the stress oscillations discussed above for $\mathrm{Co}$ on $\mathrm{Cu}(001)$. However, the apparent change of phase between MEED and stress oscillations here is due to the compressive film stress. Incomplete layer filling leads to smaller stress, indicated by a reduced magnitude of the slope of the stress curve. Thus, filled layers are identified by a pronounced negative slope of the stress curve, leading to the coincidence between stress minima with high MEED intensity. We conclude that also here the incomplete layer filling gives rise to a reduced magnitude of stress

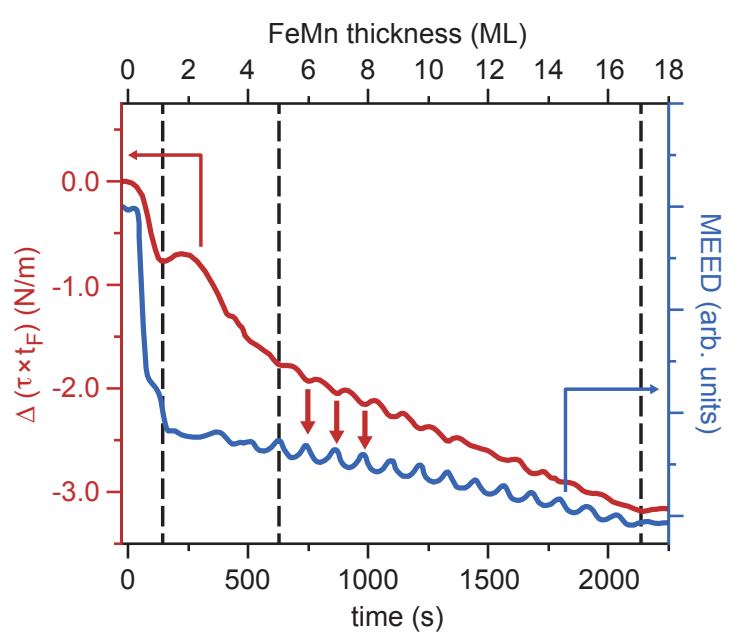

Figure 44: Simultaneously measured stress (red curve, left scale) and MEED intensity (blue curve, right scale) during the co-deposition of $\mathrm{Fe}$ and $\mathrm{Mn}$ onto $\mathrm{Cu}(001)$ at $300 \mathrm{~K}$. 202] The overall negative slope of the stress curve in films thicker than $5 \mathrm{ML}$ is ascribe dot to the compressive misfit between FeMn and $\mathrm{Cu}(001)$. Both stress and MEED intensity show monolayer oscillations in films thicker than 5 ML. Mn-Cu surface alloy formation and FeMn film formation lead to a distinctly different stress and MEED behavior in the first and for $1-5$ layers of deposition, respectively.

exerted by the FeMn islands on the substrate. Here, we have presently no STM data to confirm the presence of $\mathrm{nm}$ small FeMn islands independently. But the phenomena of stress oscillations is believed to be general, and we propose that structural relaxations in islands are also the decisive aspect for the observation of the stress oscillations in FeMn.

\section{Summary and conclusions}

To draw a bottom-line, mesoscopic misfit arising at $=$ surfaces due to both the natural misfit of materials and the reduced effective coordination has profound implications for the structure of the surface as well as growth dynamics and morphology of epitaxial structures thereon. The various ways in which misfit can affect those properties have, over the years, been minutely studied by theoretical and experimental means: bond-length contraction, in- and out-of-plane relaxations at the edges, structure-size dependent shape transitions are but a few examples. All those effects can be traced back to the influence of the misfit on the truly atomic scale on the elementary processes of atomic energetics: adsorption and diffusion.

As can be expected, geometry is not the sole property of metal-epitaxial systems affected by misfit. Electronic and magnetic properties are equally affected by mesoscopic strain relaxations, which can be traced in such properties of nanostructures, as local magnetic moments and electronic structure.

In the present review we have endeavored to outline, in a structured manner, the elementary processes in metalepitaxial systems that are susceptible to mesoscopic misfit 
(a)

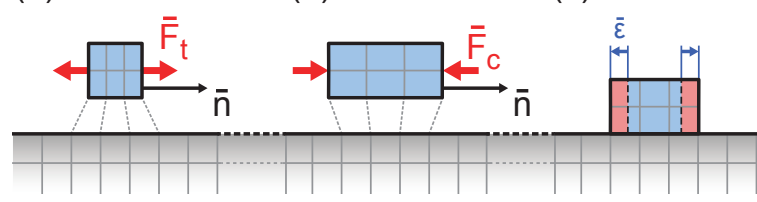

Figure A.45: Visual demonstration of the definitions of tensile (a) and compressive (b) stresses exerted on an adsorbed structure by the surface and the resulting strain induced in the structure (c).

as well as to give examples of how macro- and microscopic properties of the latter systems can thereby be affected. Starting from theoretical predictions of mesoscopic misfit we have recalled experimental observations of its consequences for structure, electronic and magnetic properties of epitaxial systems, rounding them up by presenting experimental techniques (cantilever stress measurement, surface $\mathrm{x}$-ray diffraction and extended x-ray absorption fine structure microscopies) allowing one to directly and unambiguously observe mesoscopic misfit in metal-epitaxial systems.

\section{Acknowledgement}

The authors thank Artemiy N. Baranov, Marco Corbetta, Daryl Crozier, Lars Diekhöner, Roberto Felici, Robert Gordon, Hawoong Hong, Pavel A. Ignatiev, Andrey Klavsyuk, Oksana Lysenko, Oleg Mironets, Katayoon Mohseni, Nikolay N. Negulyaev, Larissa Niebergall, Hirofumi Oka, Safia Ouazi, Wei Pan, Soo-Hyon Phark, Štěpán Pick, Jörg Premper, Mircea V. Rastei, Dmitry Tsivlin, Paul Zschack as well as Patrick Bruno, Jean-Pierre Bucher, Peter Dederichs, Wolfram Hergert, Klaus Kern for their contributions to the results presented here. We thank Nicole Kuroowsky, Wolfgang Greie and Frank Weiss for expert technical support. One of the authors (HLM) thanks the staff of the European Synchrotron Radiation Facility, and the staff of the Advanced Light Source for their hospitality during his visit in Grenoble and Argonne. This work is supported by the DFG through SFB 762. Work at the APS is supported by the U.S. DOE under Contract No. DE-AC02-06CH11357. One of the authors (DIB) thanks the Russian Foundation of Basic Research (RFBR) for financial support.

\section{Appendix A. Stress and strain defined}

While in the present review we do not intend to present a didactic definition of stress, strain, interfacial pressures etc., referring the reader to her/his favorite college book on classical mechanics, we shall briefly define certain term that are used throughout the bulk of the present review.

Let us start of by defining "stress". Pertaining to adstructures on surfaces, we shall speak of stress as a measure of strength of the forces acting on an ad-structure as it is brought into contact with the surface it is to be adsorbed on[see Fig. A.45(a,b)]. Ignoring for now the microscopic origins of those forces, in macroscopic mechanics, the stress is called "compressive", if the projection of the force $\vec{F}$ acting on an element of a surface onto that element's normal $\vec{n}$ pointing outward from the object is negative, i.e. the stress seeks to locally contract the object [Fig. A.45(a)]. Stress induced by the forces with a positive projection onto the surface normal is called "tensile". [Fig. A.45(b)]. The elastic deformation of the body/object resulting from compressive or tensile stresses is called a "strain" [denoted $\varepsilon$ in Fig. A.45(c)].

One thing which has to be mentioned at this point is that the reader should not be mislead by the fact that in Fig. A.45(c) the substrate seems to be rigid and unaffected by the adstructure. In reality, of course, since the forces exerted on the ad-structure by the substrate are reciprocated by the forces of the same strength and opposite direction being exerted onto the surface by the ad-structure. Thus surfaces also experiencing stress, which leads to a deformation of the surface, i.e. substrate strain.

While in macroscopic mechanics it is convenient to operate with shape and position of finite or infinite elements of an object to describe its deformation and forces exerted thereon, in our micro- or meso-scopic view it might be easier to think in terms of interatomic distances or bond lengths. Thus we can call a stress acting on an ad-structure compressive or tensile depending on whether it seeks to contract or stretch the interatomic bonds within the nanostructure. In reality, the forces/stress exerted onto the nano-structure by the substrate can be extremely complex and inhomogeneous owing to the numerous micros coping physical mechanisms and aspects of the latter two, often resulting in intricate strain fields, as briefly mentioned in the introductory section of the present review.

[1] J. W. Gibbs, The Collected Works of J. Willard Gibbs. Vol. 1: Thermodynamics, 3rd Edition, Yale University Press, New Haven, 1957.

[2] F. G. Donnan, A. E. Haas, A commentary on the scientific writings of J. Willard Gibbs. Yale University Press;, New Haven,, 1936.

[3] L. Royer, Recherches expérimentales sur l'épitaxie ou orientation mutuelle de cristaux d'espèces differentes, Bull. Soc. franç. Minér. Cris. 51 (1928) 7.

[4] G. Wulff, Zur Frage der Geschwindigkeit des Wachstums und der Auflösung der Krystallflachen, Z. Krist. Miner. 34 (1901) 449.

[5] C. Herring, The Use of Classical Macroscopic Concepts in Surface Energy Problems, in: R. Gomer, C. S. Smith (Eds.), Struct. Prop. Solid Surfaces, University of Chicago Press, 1953, pp. $5-81$.

[6] R. Shuttleworth, The Surface Tension of Solids Proc. Phys. Soc. Sect. A 63 (5) (1950) 444-457.

[7] P. Nozières, D. E. Wolf, Interfacial properties of elastically strained materials, Zeitschrift für Phys. B Condens. Matter 70 (3) (1988) 399-407.

[8] S. Jain, W. Hayes, Structure, properties and applications of GexSi'1-x strained layers and superlattices, Semicond. Sci. Technol. 6 (7) (1991) 547-576.

[9] D. Sander, R. Skomski, A. Enders, C. Schmidthals, D. Reuter, J. Kirschner, The correlation between mechanical stress and magnetic properties of ultrathin films, J. Phys. D. Appl. Phys. 31 (6) (1998) 663-670.

[10] D. Sander, The correlation between mechanical stress and 
magnetic anisotropy in ultrathin films Reports Prog. Phys. 62 (5) (1999) 809-858.

[11] C. Davisson, L. H. Germer, The Scattering of Electrons by a Single Crystal of Nickel Nature 119 (2998) (1927) 558-560.

[12] J. E. Lennard-Jones, B. M. Dent, The change in lattice spacing at a crystal boundary Proc. R. Soc. London 121 (787) (1928) $247-259$.

[13] C. Davisson, L. Germer, Reflection of Electrons by a Crystal of Nickel, Proc. Natl. Acad. Sci. U. S. A. 14 (4) (1928) 317.

[14] C. Davisson, L. Germer, Reflection and Refraction of Electrons by a Crystal of Nickel, Proc. Natl. Acad. Sci. U. S. A. 14 (8) (1928) 619-627

[15] H. Bethe, Über die Streuung von Elektronen an Krystallen, Naturwissenschaften 15 (1927) 787.

[16] H. Bethe, Die Streuung von Elektronen an Krystallen, Naturwissenschaften 16 (1928) 333.

[17] L. Pauling, The Principles Determining the Structure of Complex Ionic Crystals J. Am. Chem. Soc. 51 (4) (1929) 10101026.

[18] L. Pauling, The Nature of The Chemical Bond. Aplication of Results Obtained From the Quantum Mechanics and from a Theory of Paramagnetic Susceptibility to the Structure of Molecules J. Am. Chem. Soc. 53 (4) (1931) 1367-1400.

[19] L. Pauling, Atomic Radii and Interatomic Distances in Metals, J. Am. Chem. Soc. 69 (3) (1947) 542-553.

[20] F. W. C. Boswell, Precise Determination of Lattice Constants by Electron Diffraction and Variations in the Lattice Constants of Very Small Crystallites, Proc. Phys. Soc. Sect. A 64 (5) (1951) 465-476

[21] C. Berry, Electron Diffraction from Small Crystals, Phys. Rev. 88 (3) (1952) 596-599.

[22] T. B. Rymer, K. H. R. Wright, A New Determination of h/sqrt(em 0) by Electron Diffraction Proc. R. Soc. A Math. Phys. Eng. Sci. 215 (1123) (1952) 550-564.

[23] M. M. Nicolson, Surface Tension in Ionic Crystals, Proc. R. Soc. A Math. Phys. Eng. Sci. 228 (1175) (1955) 490-510.

[24] C. Mays, J. Vermaak, D. Kuhlmann-Wilsdorf, On surface stress and surface tension: II. Determination of the surface stress of gold, Surf. Sci. 12 (2) (1968) 134-140

[25] J. Vermaak, C. Mays, D. Kuhlmann-Wilsdorf, On surface stress and surface tension: I. Theoretical considerations, Surf. Sci. 12 (2) (1968) 128-133.

[26] G. Binnig, H. Rohrer, Scanning tunneling microscopy-from birth to adolescence, Rev. Mod. Phys. 59 (3) (1987) 615-625.

[27] K. Wandelt, Properties and influence of surface defects, Surf. Sci. 251-252 (1991) 387-395.

[28] J. Tersoff, R. Tromp, Shape transition in growth of strained islands: Spontaneous formation of quantum wires Phys. Rev. Lett. 70 (18) (1993) 2782-2785.

[29] J. Massies, N. Grandjean, Oscillation of the lattice relaxation in layer-by-layer epitaxial growth of highly strained materials Phys. Rev. Lett. 71 (9) (1993) 1411-1414.

[30] J. Fassbender, U. May, B. Schirmer, R. Jungblut, B. Hillebrands, G. Güntherodt, Oscillatory Surface In-Plane Lattice Spacing during Growth of $\mathrm{Co}$ and of $\mathrm{Cu}$ on a $\mathrm{Cu}(001)$ Single Crystal Phys. Rev. Lett. 75 (24) (1995) 4476-4479.

[31] B. Müller, B. r. Fischer, L. Nedelmann, A. Fricke, K. Kern, Strain Relief at Metal Interfaces with Square Symmetry, Phys. Rev. Lett. 76 (13) (1996) 2358-2361.

[32] D. Menzel, Adsorbate-Induced Global and Local Expansions and Contractions of a Close-Packed Transition Metal Surface Surf. Rev. Lett. 04 (06) (1997) 1283-1289.

[33] E. Bauer, Phänomenologische Theorie der Kristallabscheidung an Oberflächen. I Zeitschrift für Krist. 110 (1-6) (1958) 372394.

[34] H. Brune, M. Giovannini, K. Bromann, K. Kern, Self-organized growth of nanostructure arrays on strain-relief patterns, Nature 394 (July) (1998) 451-453.

[35] B. Müller, L. Nedelmann, B. r. Fischer, H. Brune, J. Barth, K. Kern, Island Shape Transition in Heteroepitaxial Metal Growth on Square Lattices Phys. Rev. Lett. 80 (12) (1998)
2642-2645.

[36] V. S. Stepanyuk, D. I. Bazhanov, A. N. Baranov, W. Hergert, P. Dederichs, J. Kirschner, Strain relief and island shape evolution in heteroepitaxial metal growth Phys. Rev. B 62 (23) (2000) 15398-15401.

[37] O. Lysenko, V. Stepanyuk, W. Hergert, J. Kirschner, Mesoscopic Relaxation in Homoepitaxial Metal Growth, Phys. Rev. Lett. 89 (12) (2002) 126102.

[38] N. Levanov, V. Stepanyuk, W. Hergert, D. Bazhanov, P. Dederichs, A. Katsnelson, C. Massobrio, Energetics of Co adatoms on the $\mathrm{Cu}(001)$ surface, Phys. Rev. B 61 (3) (2000) 2230-2234.

[39] S. M. Hu, Film-edge-induced stress in substrates, J. Appl. Phys. 50 (7) (1979) 4661.

[40] D. Sander, C. Schmidthals, A. Enders, J. Kirschner, Stress and structure of Ni monolayers on W(110): The importance of lattice mismatch, Phys. Rev. B 57 (3) (1998) 1406-1409.

[41] R. Kern, P. Müller, Elastic relaxation of coherent epitaxial deposits Surf. Sci. 392 (1-3) (1997) 103-133.

[42] P. Turban, L. Hennet, S. Andrieu, In-plane lattice spacing oscillatory behaviour during the two-dimensional hetero- and homoepitaxy of metals, Surf. Sci. 446 (3) (2000) 241-253.

[43] V. Stepanyuk, D. Tsivlin, D. Sander, W. Hergert, J. Kirschner, Mesoscopic scenario of strain-relief at metal interfaces, Thin Sol. Film. 428 (1-2) (2003) 1-5.

[44] D. Tsivlin, V. Stepanyuk, W. Hergert, J. Kirschner, Effect of mesoscopic relaxations on diffusion of Co adatoms on $\mathrm{Cu}(111)$, Phys. Rev. B 68 (20) (2003) 205411.

[45] R. Dana, Y. Manassen, Mesoscopic mismatch as a driving force for modified morphology above percolation Eur. Lett. 79 (1) (2007) 16001.

[46] W. Feng, H. L. Meyerheim, K. Mohseni, O. O. Brovko, V. S. Stepanyuk, N. Jedrecy, R. Felici, J. Kirschner, Misfit-Induced Modification of Structure and Magnetism in $\mathrm{O} / \mathrm{Fe}(001)$ p(1×1), Phys. Rev. Lett. 110 (23) (2013) 235503.

[47] M. Gsell, Effect of Substrate Strain on Adsorption Science 280 (5364) (1998) 717-720.

[48] M. Mavrikakis, B. Hammer, J. Nø rskov, Effect of Strain on the Reactivity of Metal Surfaces Phys. Rev. Lett. 81 (13) (1998) 2819-2822.

[49] V. Stepanyuk, D. Bazhanov, W. Hergert, J. Kirschner, Strain and adatom motion on mesoscopic islands, Phys. Rev. B 63 (15) (2001) 153406.

[50] D. Sekiba, Y. Yoshimoto, K. Nakatsuji, Y. Takagi, T. Iimori, S. Doi, F. Komori, Strain-induced change in electronic structure of $\mathrm{Cu}(100)$ Phys. Rev. B 75 (11) (2007) 115404.

[51] R. Miron, K. Fichthorn, Heteroepitaxial growth of $\mathrm{Co} / \mathrm{Cu}(001)$ : An accelerated molecular dynamics simulation study, Phys. Rev. B 72 (3) (2005) 035415.

[52] D. Sekiba, S. Doi, K. Nakatsuji, F. Komori, Effects of strain field in nitrogen-mediated Co film growth on $\mathrm{Cu}(001)$ : Segregation and electronic structure change Surf. Sci. $590(2-3)$ (2005) 138-145.

[53] D. Sekiba, K. Nakatsuji, Y. Yoshimoto, F. Komori, Direct Observation of Strain-Induced Change in Surface Electronic Structure, Phys. Rev. Lett. 94 (1) (2005) 016808.

[54] J. V. Barth, G. Costantini, K. Kern, Engineering atomic and molecular nanostructures at surfaces. Nature 437 (7059) (2005) 671-9.

[55] L. Sun, M. Hohage, P. Zeppenfeld, R. Balderas-Navarro, K. Hingerl, Strain Oscillations Probed with Light Phys. Rev. Lett. 96 (1) (2006) 016105.

[56] J. Evans, P. Thiel, M. Bartelt, Morphological evolution during epitaxial thin film growth: Formation of 2D islands and 3D mounds, Surf. Sci. Rep. 61 (1-2) (2006) 1-128.

[57] T. Michely, J. Krug, Islands, Mounds and Atoms, Springer Series in Surface Sciences, Springer, Berlin, 2004.

[58] M. Rastei, J. Bucher, P. Ignatiev, V. Stepanyuk, P. Bruno, Surface electronic states in Co nanoclusters on $\mathrm{Au}(111)$ : Scanning tunneling spectroscopy measurements and ab initio calculations, Phys. Rev. B 75 (4) (2007) 045436.

[59] W. J. Huang, R. Sun, J. Tao, L. D. Menard, R. G. Nuzzo, 
J. M. Zuo, Coordination-dependent surface atomic contraction in nanocrystals revealed by coherent diffraction. Nat. Mater. 7 (4) (2008) 308-13

[60] T. Menteş, N. Stojić, N. Binggeli, M. Niño, A. Locatelli, L. Aballe, M. Kiskinova, E. Bauer, Strain relaxation in small adsorbate islands: O on W(110), Phys. Rev. B 77 (15) (2008) 155414.

[61] O. Mironets, H. Meyerheim, C. Tusche, V. Stepanyuk, E. Soyka, P. Zschack, H. Hong, N. Jeutter, R. Felici, J. Kirschner, Direct Evidence for Mesoscopic Relaxations in Cobalt Nanoislands on $\mathrm{Cu}(001)$ Phys. Rev. Lett. 100 (9) (2008) 096103.

[62] N. Negulyaev, V. Stepanyuk, P. Bruno, L. Diekhöner, P. Wahl, K. Kern, Bilayer growth of nanoscale Co islands on $\mathrm{Cu}(111)$ Phys. Rev. B 77 (12) (2008) 125437.

[63] C. Becker, K. Wandelt, Surfaces: two-dimensional templates. Top. Curr. Chem. 287 (October 2008) (2009) 45-86.

[64] Y. Nahas, V. Repain, C. Chacon, Y. Girard, J. Lagoute, G. Rodary, J. Klein, S. Rousset, H. Bulou, C. Goyhenex, Dominant Role of the Epitaxial Strain in the Magnetism of Core-Shell $\mathrm{Co} / \mathrm{Au}$ Self-Organized Nanodots, Phys. Rev. Lett. 103 (6) (2009) 067202.

[65] N. Negulyaev, V. Stepanyuk, L. Niebergall, P. Bruno, W. Auwärter, Y. Pennec, G. Jahnz, J. Barth, Effect of strain relaxations on heteroepitaxial metal-on-metal island nucleation and superlattice formation: $\mathrm{Fe}$ on $\mathrm{Cu}(111)$, Phys. Rev. B 79 (19) (2009) 195411

[66] K. Bobrov, L. Guillemot, Strain engineering tunable nanotemplates on the $\mathrm{Cu}(110)-(2 \times 1)-\mathrm{O}$ surface Surf. Sci. $604(21-22)$ (2010) 1894-1898.

[67] F. Donati, G. Fratesi, M. Passoni, C. Casari, A. Mairov, C. Bottani, M. I. Trioni, A. Li Bassi, Strain effect on local electronic properties of $\mathrm{Fe}$ nanoislands grown on $\mathrm{Au}(111)$, Phys. Rev. B 83 (15) (2011) 153404.

[68] D. Sander, J. Kirschner, Non-linear magnetoelastic coupling in monolayers: Experimental challenges and theoretical insights Phys. Status Solidi 248 (10) (2011) 2389-2397.

[69] S. Ouazi, S. Wedekind, G. Rodary, H. Oka, D. Sander, J. Kirschner, Magnetization Reversal of Individual Co Nanoislands Phys. Rev. Lett. 108 (10) (2012) 107206.

[70] P. M. Diehm, P. Ágoston, K. Albe, Size-dependent lattice expansion in nanoparticles: reality or anomaly?, Chemphyschem 13 (10) (2012) 2443-54

[71] D. Sander, H. Oka, M. Corbetta, V. Stepanyuk, J. Kirschner, New insights into nano-magnetism by spin-polarized scanning tunneling microscopy J. Electron Spectros. Relat. Phenomena 189 (2013) 206-215

[72] K. Wandelt (Ed.), Solid-Solid Interfaces and Thin Films, Vol. 4, WILEY-VCH Verlag GmbH \& Co. KGaA, 2013.

[73] D. Sander, R. Skomski, C. Schmidthals, A. Enders, J. Kirschner, Film Stress and Domain Wall Pinning in Sesquilayer Iron Films on W(110). Phys. Rev. Lett. 77 (12) (1996) 2566-2569.

[74] A. Grossmann, W. Erley, J. Hannon, H. Ibach, Giant Surface Stress in Heteroepitaxial Films: Invalidation of a Classical Rule in Epitaxy. Phys. Rev. Lett. 77 (1) (1996) 127-130.

[75] X. W. Zhou, H. N. G. Wadley, Atomistic simulations of the vapor deposition of $\mathrm{Ni} / \mathrm{Cu} / \mathrm{Ni}$ multilayers: The effects of adatom incident energy J. Appl. Phys. 84 (4) (1998) 2301.

[76] B. Heinrich, J. Cochran, M. Kowalewski, J. Kirschner, Z. Celinski, A. Arrott, K. Myrtle, Magnetic anisotropies and exchange coupling in ultrathin fcc $\mathrm{Co}(001)$ structures Phys. Rev. B 44 (17) (1991) 9348-9361

[77] W. Pan, R. Popescu, H. Meyerheim, D. Sander, O. Robach, S. Ferrer, M.-T. Lin, J. Kirschner, Stress and structure of c(2× 2) and $\mathrm{p} 2 \mathrm{gg}(4 \times 2) \mathrm{Mn} / \mathrm{Cu}(001)$ surface alloys Phys. Rev. B 71 (17) (2005) 174439.

[78] R. Pentcheva, M. Scheffler, Stable and metastable structures of $\mathrm{Co}$ on $\mathrm{Cu}(001)$ : An ab initio study Phys. Rev. B 61 (3) (2000) 2211-2220.

[79] L. Shilkrot, D. Srolovitz, Elastic field of a surface step: Atom- istic simulations and anisotropic elastic theory. Phys. Rev. B. Condens. Matter 53 (16) (1996) 11120-11127.

[80] W. Yu, A. Madhukar, Molecular Dynamics Study of Coherent Island Energetics, Stresses, and Strains in Highly Strained Epitaxy, Phys. Rev. Lett. 79 (5) (1997) 905-908.

[81] H. Müller, H.-G. Fritsche, L. Skala, Analytic Cluster Models and Interpolation Formulae for Cluster Properties in: H. Haberland (Ed.), Clust. Atoms Mol., Vol. 52 of Springer Series in Chemical Physics, Springer Berlin Heidelberg, Berlin, Heidelberg, 1994, p. 114

[82] H. Bulou, F. Scheurer, P. Ohresser, A. Barbier, S. Stanescu, C. Quirós, Structure of self-organized Fe clusters grown on $\mathrm{Au}(111)$ analyzed by grazing incidence x-ray diffraction. Phys. Rev. B 69 (15) (2004) 155413

[83] M. Mundschau, E. Bauer, W. Telieps, W. Święch, In situ studies of epitaxial growth in the low energy electron microscope, Surf. Sci. 213 (2-3) (1989) 381-392.

[84] S. Rousset, S. Chiang, D. E. Fowler, D. D. Chambliss, Intermixing and three-dimensional islands in the epitaxial growth of $\mathrm{Au}$ on $\mathrm{Ag}(110)$ Phys. Rev. Lett. 69 (22) (1992) 3200-3203.

[85] H.-C. Jeong, E. D. Williams, Steps on surfaces: experiment and theory, Surf. Sci. Rep. 34 (6-8) (1999) 171-294.

[86] A. Li, F. Liu, M. Lagally, Equilibrium shape of twodimensional islands under stress, Phys. Rev. Lett. 85 (9) (2000) 1922-5.

[87] H. Brune, Microscopic view of epitaxial metal growth: Nucleation and aggregation Surf. Sci. Rep. 31 (3-4) (1998) 121-229.

[88] A. Gölzhäuser, G. Ehrlich, Atom Movement and Binding on Surface Clusters: Pt on Pt(111) Clusters, Phys. Rev. Lett. 77 (7) (1996) 1334-1337.

[89] S. C. Wang, G. Ehrlich, Atom incorporation at surface clusters: An atomic view, Phys. Rev. Lett. 67 (18) (1991) 2509-2512.

[90] S. C. Wang, G. Ehrlich, Adatom motion to lattice steps: A direct view, Phys. Rev. Lett. 70 (1) (1993) 41-44.

[91] O. O. Brovko, N. N. Negulyaev, V. S. Stepanyuk, Relativistic peculiarities at stepped surfaces: Energetics and diffusion patterns obtained from ab initio calculations, Phys. Rev. B 82 (15) (2010) 155452.

[92] B. Yu, M. Scheffler, Physical origin of exchange diffusion on fcc(100) metal surfaces Phys. Rev. B 56 (24) (1997) R15569R15572.

[93] H. Brune, K. Bromann, H. Röder, K. Kern, J. Jacobsen, P. Stoltze, K. Jacobsen, J. No/rskov, Effect of strain on surface diffusion and nucleation. Phys. Rev. B 52 (20) (1995) R14380R14383.

[94] C. Ratsch, A. P. Seitsonen, M. Scheffler, Strain dependence of surface diffusion: $\mathrm{Ag}$ on $\mathrm{Ag}(111)$ and $\mathrm{Pt}(111)$, Phys. Rev. B 55 (11) (1997) 6750-6753.

[95] M. Schroeder, D. Wolf, Diffusion on strained surfaces, Surf. Sci. 375 (1) (1997) 129-140.

[96] R. Sabiryanov, M. Larsson, K. Cho, W. Nix, B. Clemens, Surface diffusion and growth of patterned nanostructures on strained surfaces, Phys. Rev. B 67 (12) (2003) 125412.

[97] M. Breeman, G. Rosenfeld, G. Comsa, Mechanism for island formation during low-temperature growth on (100) surfaces of fcc metals Phys. Rev. B 54 (23) (1996) 16440-16443.

[98] S. Wang, G. Ehrlich, Atom condensation at lattice steps and clusters, Phys. Rev. Lett. 71 (25) (1993) 4174-4177.

[99] Y. He, E. Borguet, Effect of local environment on nanoscale dynamics at electrochemical interfaces: Anisotropic growth and dissolution in the presence of a step providing evidence for a Schwoebel-Ehrlich barrier at solid/liquid interfaces Faraday Discuss. 121 (2002) 17-25.

[100] H. Brune, Creating Metal Nanostructures at Metal Surfaces Using Growth Kinetics, in: E. Hasselbrink, B. I. Lundqvist (Eds.), Handb. Surf. Sci. Vol. 3, Dyn., Vol. 3, Elsevier, 2008, Ch. 15 , pp. $761-786$.

[101] Y. Li, A. E. DePristo, Potential energy barriers for interlayer mass transport in homoepitaxial growth on fcc(111) surfaces: Pt and Ag Surf. Sci. 319 (1-2) (1994) 141-148.

[102] O. Lysenko, V. Stepanyuk, W. Hergert, J. Kirschner, Interlayer 
mass transport in homoepitaxy on the atomic scale Phys. Rev. B 68 (3) (2003) 033409

[103] K. Bromann, H. Brune, H. Röder, K. Kern, Interlayer Mass Transport in Homoepitaxial and Heteroepitaxial Metal Growth Phys. Rev. Lett. 75 (4) (1995) 677-680.

[104] M. Villarba, H. Jónsson, Diffusion mechanisms relevant to metal crystal growth: Pt/Pt(111), Surf. Sci. 317 (1-2) (1994) 15-36.

[105] M. Giesen, G. Schulze Icking-Konert, H. Ibach, Fast Decay of Adatom Islands and Mounds on $\mathrm{Cu}(111)$ : A New Effective Channel for Interlayer Mass Transport Phys. Rev. Lett. 80 (3) (1998) 552-555.

[106] M. Giesen, G. Schulze Icking-Konert, H. Ibach, Interlayer Mass Transport and Quantum Confinement of Electronic States Phys. Rev. Lett. 82 (15) (1999) 3101-3104.

[107] M. Giesen, H. Ibach, On the mechanism of rapid mound decay Surf. Sci. 464 (1) (2000) L697-L702.

[108] K. Morgenstern, G. Rosenfeld, G. Comsa, M. Sø rensen, B. r. Hammer, E. Læ gsgaard, F. Besenbacher, Kinetics of fast island decay on $\mathrm{Ag}(111)$, Phys. Rev. B 63 (4) (2001) 045412.

[109] S. Jay Chey, L. Huang, J. Weaver, Self-assembly of multilayer arrays from $\mathrm{Ag}$ nanoclusters delivered to $\mathrm{Ag}(111)$ by soft landing Surf. Sci. 419 (1) (1998) L100-L106.

[110] R. Longo, V. Stepanyuk, W. Hergert, A. Vega, L. Gallego, J. Kirschner, Interface intermixing in metal heteroepitaxy on the atomic scale Phys. Rev. B 69 (7) (2004) 073406.

[111] K. Johnson, D. Chambliss, R. Wilson, S. Chiang, A structural model and mechanism for Fe epitaxy on $\mathrm{Cu}(100)$ Surf. Sci. 313 (3) (1994) L811-L816.

[112] D. Chambliss, K. Johnson, Nucleation with a critical cluster size of zero: Submonolayer Fe inclusions in $\mathrm{Cu}(100)$, Phys. Rev. B 50 (7) (1994) 5012-5015.

[113] S. Brongersma, M. Castell, D. Perovic, M. Zinke-Allmang, Stress-Induced Shape Transition of CoSi2 Clusters on Si(100) Phys. Rev. Lett. 80 (17) (1998) 3795-3798.

[114] W. Ma, R. Nötzel, H.-P. Schönherr, K. H. Ploog, Shape transition of coherent three-dimensional (In, Ga)As islands on GaAs(100) Appl. Phys. Lett. 79 (25) (2001) 4219.

[115] B. Müller, L. P. Nedelmann, B. r. Fischer, H. Brune, J. V. Barth, K. Kern, D. Erdös, J. Wollschläger, Strain Relief via Island Ramification in Submonolayer Hereroepitaxy, Surf. Rev. Lett. 05 (03n04) (1998) 769-781.

[116] B. Müller, Natural formation of nanostructures: from fundamentals in metal heteroepitaxy to applications in optics and biomaterials sciences, Surf. Rev. Lett. 08 (01n02) (2001) 169228.

[117] Y. Shim, J. G. Amar, Shape Transitions in Strained Cu Islands on $\mathrm{Ni}(100)$ : Kinetics versus Energetics, Phys. Rev. Lett. 108 (7) (2012) 076102.

[118] J. Tersoff, Surface-Confined Alloy Formation in Immiscible Systems Phys. Rev. Lett. 74 (3) (1995) 434-437.

[119] C. Zimmermann, M. Yeadon, K. Nordlund, J. Gibson, R. Averback, U. Herr, K. Samwer, Burrowing of Co Nanoparticles on Clean $\mathrm{Cu}$ and Ag Surfaces Phys. Rev. Lett. 83 (6) (1999) 1163-1166.

[120] S. Padovani, F. Scheurer, J. P. Bucher, Burrowing selforganized cobalt clusters into a gold substrate, Europhys. Lett. 45 (3) (1999) 327-333.

[121] V. Stepanyuk, D. Tsivline, D. Bazhanov, W. Hergert, A. Katsnelson, Burrowing of Co clusters on the $\mathrm{Cu}(001)$ surface: Atomic-scale calculations, Phys. Rev. B 63 (23) (2001) 235406.

[122] F. Meier, K. von Bergmann, P. Ferriani, J. Wiebe, M. Bode, K. Hashimoto, S. Heinze, R. Wiesendanger, Spin-dependent electronic and magnetic properties of Co nanostructures on $\mathrm{Pt}(111)$ studied by spin-resolved scanning tunneling spectroscopy Phys. Rev. B 74 (19) (2006) 195411.

[123] P. Grütter, U. Dürig, Growth of vapor-deposited cobalt films on Pt(111) studied by scanning tunneling microscopy Phys. Rev. B 49 (3) (1994) 2021-2029.

[124] E. Lundgren, B. Stanka, M. Schmid, P. Varga, Thin films of Co on Pt(111): Strain relaxation and growth, Phys. Rev. B
62 (4) (2000) 2843-2851.

[125] T. Cren, S. Rusponi, N. Weiss, M. Epple, H. Brune, Oxidation Induced Enhanced Magnetic Susceptibility of Co Islands on $\operatorname{Pt}(111) \dagger$ J. Phys. Chem. B 108 (38) (2004) 14685-14691.

[126] F. Donati, A. Mairov, C. Casari, M. Passoni, A. Li Bassi, Nucleation and growth mechanisms of Fe on $\mathrm{Au}(111)$ in the sub-monolayer regime Surf. Sci. 606 (7-8) (2012) 702-710.

[127] J. Barth, H. Brune, G. Ertl, R. Behm, Scanning tunneling microscopy observations on the reconstructed $\mathrm{Au}(111)$ surface: Atomic structure, long-range superstructure, rotational domains, and surface defects, Phys. Rev. B 42 (15) (1990) 9307-9318.

[128] M. Hanbücken, J.-P. Deville (Eds.), Stress and Strain in Epitaxy: Theoretical Concepts, Measurements and Applications, 2nd Edition, Elsevier, Amsterdam, 2001.

[129] H. L. Meyerheim, E. Crozier, R. Gordon, Q. Xiao, K. Mohseni, N. N. Negulyaev, V. S. Stepanyuk, J. Kirschner, Direct proof of mesoscopic misfit in nanoscale islands by x-ray absorption spectroscopy, Phys. Rev. B 85 (12) (2012) 125405.

[130] M. Rastei, B. Heinrich, L. Limot, P. Ignatiev, V. Stepanyuk, P. Bruno, J. Bucher, Size-Dependent Surface States of Strained Cobalt Nanoislands on $\mathrm{Cu}(111)$ Phys. Rev. Lett. 99 (24) (2007) 246102.

[131] D. Sander, S.-H. Phark, M. Corbetta, J. Fischer, H. Oka, J. Kirschner, The impact of structural relaxation on spinpolarization and magnetic reversal of individual nano structures studied by spin-polarized scanning tunneling microscopy, J. Phys. Condens. Matter. vv (2014) ppp.

[132] L. Diekhöner, M. A. Schneider, A. N. Baranov, V. S. Stepanyuk, P. Bruno, K. Kern, Surface States of Cobalt Nanoislands on $\mathrm{Cu}(111)$, Phys. Rev. Lett. 90 (23) (2003) 236801.

[133] O. Pietzsch, S. Okatov, A. Kubetzka, M. Bode, S. Heinze, A. Lichtenstein, R. Wiesendanger, Spin-Resolved Electronic Structure of Nanoscale Cobalt Islands on $\mathrm{Cu}(111)$ Phys. Rev. Lett. 96 (23) (2006) 237203.

[134] J. R. Cerda, P. L. de Andres, A. Cebollada, R. Miranda, E. Navas, P. Schuster, C. M. Schneider, J. Kirschner, Epitaxial growth of cobalt films on $\mathrm{Cu}(100)$ : a crystallographic LEED determination J. Phys. Condens. Matter 5 (14) (1993) 2055-2062.

[135] H. Ibach, The role of surface stress in reconstruction, epitaxial growth and stabilization of mesoscopic structures, Surf. Sci. Rep. 29 (5-6) (1997) 195-263.

[136] D. Sander, Surface stress: implications and measurements, Curr. Opin. Solid State Mater. Sci. 7 (1) (2003) 51-57.

[137] C. Kittel, Physical Theory of Ferromagnetic Domains Rev. Mod. Phys. 21 (4) (1949) 541-583.

[138] D. Sander, The magnetic anisotropy and spin reorientation of nanostructures and nanoscale films J. Phys. Condens. Matter 16 (20) (2004) R603-R636.

[139] O. Pietzsch, A. Kubetzka, M. Bode, R. Wiesendanger, Spin-Polarized Scanning Tunneling Spectroscopy of Nanoscale Cobalt Islands on $\mathrm{Cu}(111)$, Phys. Rev. Lett. 92 (5) (2004) 057202 .

[140] E. F. Kneller, F. E. Luborsky, Particle Size Dependence of Coercivity and Remanence of Single-Domain Particles J. Appl. Phys. 34 (3) (1963) 656.

[141] E. C. Stoner, E. P. Wohlfarth, A Mechanism of Magnetic Hysteresis in Heterogeneous Alloys Philos. Trans. R. Soc. A Math. Phys. Eng. Sci. 240 (826) (1948) 599-642.

[142] S. Rusponi, T. Cren, N. Weiss, M. Epple, P. Buluschek, L. Claude, H. Brune, The remarkable difference between surface and step atoms in the magnetic anisotropy of twodimensional nanostructures. Nat. Mater. 2 (8) (2003) 546-51.

[143] v. Pick, V. Stepanyuk, A. Baranov, W. Hergert, P. Bruno, Effect of atomic relaxations on magnetic properties of adatoms and small clusters, Phys. Rev. B 68 (10) (2003) 104410.

[144] v. Pick, V. Stepanyuk, A. Klavsyuk, L. Niebergall, W. Hergert, J. Kirschner, P. Bruno, Magnetism and structure on the atomic scale: Small cobalt clusters in $\mathrm{Cu}(001)$ Phys. Rev. B 70 (22) (2004) 224419 . 
[145] K. Bohnen, K. Ho, Structure and dynamics at metal surfaces Surf. Sci. Rep. 19 (3-6) (1993) 99-120.

[146] R. Smoluchowski, Anisotropy of the Electronic Work Function of Metals Phys. Rev. 60 (9) (1941) 661-674.

[147] M. V. Hove, Atomic-scale structure: From surfaces to nanomaterials, Surf. Sci. 603 (10-12) (2009) 1301-1305.

[148] S. J. L. Billinge, I. Levin, The problem with determining atomic structure at the nanoscale. Science 316 (5824) (2007) 561-5.

[149] G. Williams, M. Pfeifer, I. Vartanyants, I. Robinson, ThreeDimensional Imaging of Microstructure in $\mathrm{Au}$ Nanocrystals Phys. Rev. Lett. 90 (17) (2003) 175501

[150] Y. Xiao, Z. Cai, Z. L. Wang, B. Lai, Y. S. Chu, An X-ray nanodiffraction technique for structural characterization of individual nanomaterials. J. Synchrotron Radiat. 12 (Pt 2) (2005) 124-8.

151] P. D. Jadzinsky, G. Calero, C. J. Ackerson, D. A. Bushnell, R. D. Kornberg, Structure of a thiol monolayer-protected gold nanoparticle at 1.1 A resolution. Science 318 (5849) (2007) 430-3.

[152] O. Mironets, H. Meyerheim, C. Tusche, V. Stepanyuk, E. Soyka, H. Hong, P. Zschack, N. Jeutter, R. Felici, J. Kirschner, Bond length contraction in cobalt nanoislands on $\mathrm{Cu}(001)$ analyzed by surface x-ray diffraction, Phys. Rev. B 79 (3) (2009) 035406

[153] A. I. Frenkel, C. W. Hills, R. G. Nuzzo, A View from the Inside: Complexity in the Atomic Scale Ordering of Supported Metal Nanoparticles, J. Phys. Chem. B 105 (51) (2001) 12689-12703.

[154] P. Eisenberger, W. Marra, X-Ray Diffraction Study of the Ge(001) Reconstructed Surface, Phys. Rev. Lett. 46 (16) (1981) 1081-1084

[155] R. Feidenhans'l, Surface structure determination by X-ray diffraction, Surf. Sci. Rep. 10 (3) (1989) 105-188.

[156] I. K. Robinson, Surface Crystallography, in: G. S. Brown, D. E. Moncton (Eds.), Handb. Synchrotron Radiat., Vol. 3 of Handbook on Synchrotron Radiation, North Holland, 1991, pp. $221-266$.

[157] I. K. Robinson, D. J. Tweet, Surface X-ray diffraction, Reports Prog. Phys. 55 (5) (1992) 599-651.

[158] M. v. Laue, Die äußere Form der Kristalle in ihrem Einflußauf die Interferenzerscheinungen an Raumgittern Ann. Phys. 418 (1) (1936) 55-68.

[159] I. Robinson, Crystal truncation rods and surface roughness Phys. Rev. B 33 (6) (1986) 3830-3836.

[160] W. F. Kuhs, Generalized atomic displacements in crystallographic structure analysis, Acta Cryst. A 48 (2) (1992) 80-98.

[161] M. J. Buerger, Kristallographie. Eine Einführung in die geometrische und röntgenographische Kristallkunde, no. 3, Walter de Gruyter Verlag Berlin, Berlin, 1978.

[162] R. Pentcheva, K. Fichthorn, M. Scheffler, T. Bernhard, R. Pfandzelter, H. Winter, Non-Arrhenius Behavior of the Island Density in Metal Heteroepitaxy: $\mathrm{Co}$ on $\mathrm{Cu}(001)$, Phys. Rev. Lett. 90 (7) (2003) 076101.

[163] F. Nouvertné, U. May, M. Bamming, A. Rampe, U. Korte, G. Güntherodt, Atomic exchange processes and bimodal initial growth of $\mathrm{Co} / \mathrm{Cu}(001)$, Phys. Rev. B 60 (20) (1999) 1438214386.

[164] J. A. Venables, G. D. T. Spiller, M. Hanbucken, Nucleation and growth of thin films Reports Prog. Phys. 47 (4) (1984) 399-459.

[165] J. A. Venables, Growth and Properties of Ultrathin Epitaxial Layers, Vol. 8 of The Chemical Physics of Solid Surfaces, Elsevier, 1997.

[166] S. Ferrer, E. Vlieg, I. Robinson, Epitaxial submonolayer cobalt films on $\mathrm{Cu}(100)$ studied by X-ray diffraction, Surf. Sci. 250 (13) (1991) L363-L367.

[167] C. Schamper, H. L. Meyerheim, W. Moritz, Resolution correction for surface X-ray diffraction at high beam exit angles, J. Appl. Crystallogr. 26 (5) (1993) 687-696.

[168] E. Vlieg, Integrated Intensities Using a Six-Circle Surface Xray Diffractometer, J. Appl. Crystallogr. 30 (5) (1997) 532-
543.

[169] S. C. Abrahams, Indicators of accuracy in structure factor measurement, Acta Crystallogr. Sect. A Cryst. Physics, Diffraction, Theor. Gen. Crystallogr. 25 (1) (1969) 165-173.

[170] O. Mironets, H. Meyerheim, C. Tusche, P. Zschack, H. Hong, N. Jeutter, R. Felici, J. Kirschner, Surface vibrations and relaxation effects in $\mathrm{Cu}(001)$ studied by x-ray diffraction, Phys. Rev. B 78 (15) (2008) 153401

[171] P. Bonanno, M. Canepa, P. Cantini, R. Moroni, L. Mattera, S. Terreni, Surfactant properties of chemisorbed oxygen in Fe/Fe(001) homoepitaxy: a He diffraction study, Surf. Sci. 454-456 (2000) 697-701.

[172] A. Picone, A. Brambilla, A. Calloni, L. Duò, M. Finazzi, F. Ciccacci, Oxygen-induced effects on the morphology of the $\mathrm{Fe}(001)$ surface in out-of-equilibrium conditions, Phys. Rev. B 83 (23) (2011) 235402.

[173] H. Huang, J. Hermanson, Bonding and magnetism of chemisorbed oxygen on Fe(001), Phys. Rev. B 32 (10) (1985) $6312-6318$.

[174] K. Legg, F. Jona, D. Jepsen, P. Marcus, Early stages of oxidation of the Fe001 surface: Atomic structure of the first monolayer. Phys. Rev. B 16 (12) (1977) 5271-5276.

[175] F. Jona, P. Marcus, Re-examination of the Fe0011 × 1-O structure Solid State Commun. 64 (5) (1987) 667-671.

[176] S. Chubb, W. Pickett, First-principles determination of giant adsorption-induced surface relaxation in $\mathrm{p}$ (1times 1$)$ $\mathrm{O} / \mathrm{Fe}(001)$ Phys. Rev. Lett. 58 (12) (1987) 1248-1251.

[177] S. S. Parihar, H. L. Meyerheim, K. Mohseni, S. Ostanin, A. Ernst, N. Jedrecy, R. Felici, J. Kirschner, Structure of $\mathrm{O} / \mathrm{Fe}(001)-\mathrm{p}(1 \times 1)$ studied by surface $\mathrm{x}$-ray diffraction, Phys. Rev. B 81 (7) (2010) 075428.

[178] F. Bisio, R. Moroni, M. Canepa, L. Mattera, R. Bertacco, F. Ciccacci, Structural versus Magnetic Properties at the Surface of Fe Films during Oxygen-Assisted Homoepitaxial Growth Phys. Rev. Lett. 83 (23) (1999) 4868-4871.

[179] R. Bertacco, F. Ciccacci, Oxygen-induced enhancement of the spin-dependent effects in electron spectroscopies of $\mathrm{Fe}(001)$, Phys. Rev. B 59 (6) (1999) 4207-4210.

[180] P. Błoński, A. Kiejna, J. Hafner, Theoretical study of oxygen adsorption at the $\mathrm{Fe}(110)$ and (100) surfaces, Surf. Sci. 590 (1) (2005) 88-100

181] M. Nývlt, F. Bisio, J. Franta, C. Gao, H. Petek, J. Kirschner, Surface Magnetism during Oxygen-Aided Fe Homoepitaxy Phys. Rev. Lett. 95 (12) (2005) 127201.

[182] A. Tange, C. L. Gao, B. Y. Yavorsky, I. V. Maznichenko, C. Etz, A. Ernst, W. Hergert, I. Mertig, W. Wulfhekel, J. Kirschner, Electronic structure and spin polarization of the $\mathrm{Fe}(001)-\mathrm{p}(1 \times 1) \mathrm{O}$ surface Phys. Rev. B 81 (19) (2010) 195410.

183] A. Winkelmann, D. Hartung, H. Engelhard, C.-T. Chiang, J. Kirschner, High efficiency electron spin polarization analyzer based on exchange scattering at FeW(001). Rev. Sci. Instrum. 79 (8) (2008) 083303

[184] J. Kirschner, D. Rebenstorff, H. Ibach, High-Resolution SpinPolarized Electron-Energy-Loss Spectroscopy and the Stoner Excitation Spectrum in Nickel Phys. Rev. Lett. 53 (7) (1984) 698-701.

[185] C. M. Schlepütz, R. Herger, P. R. Willmott, B. D. Patterson, O. Bunk, C. Brönnimann, B. Henrich, G. Hülsen, E. F. Eikenberry, Improved data acquisition in grazing-incidence X-ray scattering experiments using a pixel detector. Acta Crystallogr. A. 61 (Pt 4) (2005) 418-25.

[186] B.-K. Teo, D. C. Joy (Eds.), EXAFS Spectroscopy, Springer, Boston, MA, USA, 1981

[187] B. K. Teo, EXAFS: Basic Principles and Data Analysis, Vol. 9 of Inorganic Chemistry Concepts, Springer Berlin Heidelberg, Berlin, Heidelberg, 1986.

[188] D. C. Koningsberger, R. Prins (Eds.), X-Ray Absorption: Principles, Applications, Techniques of EXAFS, SEXAFS and XANES Wiley, New York, 1988.

[189] H. Fricke, The K-Characteristic Absorption Frequencies for the Chemical Elements Magnesium to Chromium Phys. Rep. 
16 (3) (1920) 202-215.

[190] G. Hertz, Über die Absorptionsgrenzen in der L-Serie Z. Phys. 3 (1) (1920) 19-25.

[191] R. d. L. Kronig, Zur Theorie der Feinstruktur in den Röntgenabsorptionsspektren Zeitschrift für Phys. 70 (5-6) (1931) 317-323.

[192] D. Sayers, E. Stern, F. Lytle, New Technique for Investigating Noncrystalline Structures: Fourier Analysis of the Extended X-Ray-Absorption Fine Structure, Phys. Rev. Lett. 27 (18) (1971) 1204-1207.

[193] E. Stern, B. Bunker, S. Heald, Many-body effects on extended x-ray absorption fine structure amplitudes, Phys. Rev. B 21 (12) (1980) 5521-5539.

[194] P. Citrin, Bond lengths and coordination numbers from L 2,3 edge versus K-edge surface extended x-ray-absorption fine structure Phys. Rev. B 31 (2) (1985) 700-721.

[195] T. Ressler, WinXAS: a program for X-ray absorption spectroscopy data analysis under MS-Windows., J. Synchrotron Radiat. 5 (Pt 2) (1998) 118-22.

[196] S. Zabinsky, J. Rehr, A. Ankudinov, R. Albers, M. Eller, Multiple-scattering calculations of x-ray-absorption spectra Phys. Rev. B 52 (4) (1995) 2995-3009.

[197] D. Sander, S. Ouazi, V. Stepanyuk, D. Bazhanov, J. Kirschner, Stress oscillations in a growing metal film, Surf. Sci. 512 (3) (2002) 281-286

[198] D. Sander, Z. Tian, J. Kirschner, The role of surface stress in structural transitions, epitaxial growth and magnetism on the nanoscale., J. Phys. Condens. Matter 21 (13) (2009) 134015.

[199] D. Sander, A. Enders, J. Kirschner, Anisotropic surface stress on W(110) Europhys. Lett. 45 (2) (1999) 208-214.

[200] R. Mahesh, D. Sander, S. Zharkov, J. Kirschner, Stress and growth of $\mathrm{Ag}$ monolayers on a $\mathrm{Fe}(100)$ whisker. Phys. Rev. B 68 (4) (2003) 045416.

[201] D. Sander, Z. Tian, J. Kirschner, Cantilever measurements of surface stress, surface reconstruction, film stress and magnetoelastic stress of monolayers, Sensors 8 (7) (2008) 4466-4486.

[202] W. Pan, D. Sander, M.-T. Lin, J. Kirschner, Stress oscillations and surface alloy formation during the growth of FeMn on $\mathrm{Cu}(001)$, Phys. Rev. B 68 (22) (2003) 224419

[203] R. Popescu, H. Meyerheim, D. Sander, J. Kirschner, P. Steadman, O. Robach, S. Ferrer, Surface x-ray structure analysis of periodic misfit dislocations in Fe/W(110), Phys. Rev. B 68 (15) (2003) 155421

204] V. Martin, W. Meyer, C. Giovanardi, L. Hammer, K. Heinz, Z. Tian, D. Sander, J. Kirschner, Pseudomorphic growth of Fe monolayers on $\operatorname{Ir}(001)-(1 \times 1)$ : From a fct precursor to a bct film Phys. Rev. B 76 (20) (2007) 205418.

[205] D. Sander, S. Ouazi, A. Enders, T. Gutjahr-Löser, V. S. Stepanyuk, D. I. Bazhanov, J. Kirschner, Stress, strain and magnetostriction in epitaxial films, J. Phys. Condens. Matter 14 (16) (2002) 4165-4176.

[206] G. G. Stoney, The Tension of Metallic Films Deposited by Electrolysis, Proc. R. Soc. A Math. Phys. Eng. Sci. 82 (553) (1909) 172-175.

[207] R. Martinez, W. Augustyniak, J. Golovchenko, Direct measurement of crystal surface stress, Phys. Rev. Lett. 64 (9) (1990) 1035-1038.

[208] A. Schell-Sorokin, R. Tromp, Mechanical stresses in (sub)monolayer epitaxial films, Phys. Rev. Lett. 64 (9) (1990) 1039-1042.

[209] M. Weber, R. Koch, K. Rieder, UHV Cantilever Beam Technique for Quantitative Measurements of Magnetization, Magnetostriction, and Intrinsic Stress of Ultrathin Magnetic Films Phys. Rev. Lett. 73 (8) (1994) 1166-1169.

[210] Z. Tian, D. Sander, J. Kirschner, Nonlinear magnetoelastic coupling of epitaxial layers of Fe, Co, and Ni on Ir(100) Phys. Rev. B 79 (2) (2009) 024432.

[211] J. Premper, D. Sander, J. Kirschner, A combined surface stress and magneto-optical Kerr effect measurement setup for temperatures down to $30 \mathrm{~K}$ and in fields of up to $0.7 \mathrm{~T}$. Rev. Sci. Instrum. 83 (7) (2012) 073904
[212] K. Dahmen, S. Lehwald, H. Ibach, Bending of crystalline plates under the influence of surface stress - a finite element analysis, Surf. Sci. 446 (1-2) (2000) 161-173.

[213] K. Dahmen, H. Ibach, D. Sander, A finite element analysis of the bending of crystalline plates due to anisotropic surface and film stress applied to magnetoelasticity J. Magn. Magn. Mater. 231 (1) (2001) 74-84.

[214] A. Schmid, J. Kirschner, In situ observation of epitaxial growth of Co thin films on $\mathrm{Cu}(100)$, Ultramicroscopy 42-44 (1992) 483-489.

[215] J. Fassbender, R. Allenspach, U. Dürig, Intermixing and growth kinetics of the first Co monolayers on $\mathrm{Cu}(001)$ Surf. Sci. 383 (2-3) (1997) L742-L748.

[216] A. Zangwill, Physics at surfaces, Cambridge University Press, Cambridge, 1988.

[217] J. Silveira, F. Briones, In situ observation of reconstruction related surface stress during molecular beam epitaxy (MBE) growth of III-V compounds J. Cryst. Growth 201-202 (1999) 113-117.

[218] D. Flötotto, Z. Wang, L. P. H. Jeurgens, E. J. Mittemeijer, Quantum Confinement Drives Macroscopic Stress Oscillations at the Initial Stage of Thin Film Growth, Phys. Rev. Lett. 109 (4) (2012) 045501. 